\title{
STED Nanoscopy of the Living Brain
}

\author{
Dissertation \\ zur Erlangung des mathematisch-naturwissenschaftlichen Doktorgrades \\ "Doctor rerum naturalium" \\ der Georg-August-Universität Göttingen
}

\author{
vorgelegt von \\ Sebastian Berning \\ aus Kirchheimbolanden
}

Göttingen 2011 
Prof. Dr. Stefan W. Hell (Referent)

Abt. NanoBiophotonics, MPI für Biophysikalische Chemie

Prof. Dr. Christoph F. Schmidt (Referent)

III. Physikalisches Institut - Biophysik, Universität Göttingen

Prof. Dr. Helmut Grubmüller

Abt. Theoretical and Computational Biophysics, MPI für Biophysikalische Chemie

Tag der mündlichen Prüfung: 13. Dezember 2011 
I hereby ensure that the presented thesis "STED Nanoscopy of the Living Brain" has been written independently and with no other sources and aids than quoted.

Göttingen, 15th of November 2011

Sebastian Berning 


\section{Contents}

1 Introduction 1

1.1 At the frontier of optical microscopy . . . . . . . . . . . . . . 4

1.2 Far-field optical nanoscopy . . . . . . . . . . . . . . . . 5

2 STED microscopy through planar dielectric interfaces $\quad 9$

2.1 Vectorial simulation of focal fields . . . . . . . . . . . . . . . 10

2.2 Aberrations introduced by a single dielectric interface . . . . . . . 11

2.2.1 Enhanced numerical model . . . . . . . . . . . . . . . . 12

2.2.3 2D STED microscopy in the brain with oil-immersion objectives 14

2.2.4 Experimental validation . . . . . . . . . . . . . 15

2.2.5 3D STED with an oil-immersion objective . . . . . . . . 17

2.3 Adding a glass coverslip to the model system . . . . . . . . . . 18

2.3.1 Corrected objectives with glycerol and water immersion . . . 20

2.4 Coverslip tilt . . . . . . . . . . . . . . . . . . . . . . . 21

2.4.1 Introducing tilt to the numerical model . . . . . . . . . . 22

2.4.2 Effects of coverslip tilt on 2D STED microscopy . . . . . . 23

2.4.3 Aberrations in 3D STED resulting from coverslip tilt . . . . 25

2.5 Summary . . . . . . . . . . . . . . . . . 26

3 Experimental methods and techniques 27

3.1 A STED microscope for physiology applications . . . . . . . . . . 27

3.2 Dual-color STED imaging with EGFP and EYFP . . . . . . . . . . . 29

3.3 Experimental procedure . . . . . . . . . . . . . . 33

3.3.1 Anesthesia and surgery . . . . . . . . . . . . . 33

3.3.2 Maintenance of the mouse . . . . . . . . . . . . 35

3.3.3 Image acquisition and analysis . . . . . . . . . 35

4 Experimental results $\quad 37$

4.1 Dendritic spine plasticity in the adult brain . . . . . . . . . . . . . 37

4.2 Microanatomy of oligodendroglia resolved in vivo . . . . . . . . . . 41

4.3 Dual-color STED microscopy of neurons and astrocytes . . . . . . . . 45

5 Conclusion and Outlook 48

Bilbliography 51

$\begin{array}{ll}\text { Appendix } & 61\end{array}$ 
Es que, realmente, dejando aparte los halagos del amor propio, el jardín de la neurología brinda al investigador espectáculos cautivadores y emociones artísticas incomparables. En él hallaron, al fin, mis instintos estéticos plena satisfacción.

¡Como el entomólogo a caza de mariposas de vistosos matices, mi atención perseguía,

en el vergel de la substancia gris, células de formas delicadas y elegantes, las misteriosas mariposas del alma, cuyo batir de alas quién sabe si esclarecerá algún día el secreto de la vida mental!

("Really, the garden of neurology offers the researcher captivating spectacles and incomparable artistic emotions. My aesthetic instincts find their full satisfaction. Like the entomologist catching beautiful butterflies, my attention pursued in the garden of gray matter, the delicately and gracefully shaped cells, the mysterious butterflies whose wing beats might someday reveal the secret of mental life.") 


\section{Introduction}

When the young Spanish histologist and artist Santiago Ramón y Cajal set off to "reveal the secret of mental life in the garden of gray matter" ${ }^{\prime \prime}$ in the second half of the $19^{\text {th }}$ century, very little was known about the microscopic structure of the most complex organ of the body. While contemporary encyclopedic volumes of neuroanatomical knowledge revealed detailed and accurate descriptions of all of the peripheral nerves [3], their trace was quickly lost where they fused with the seemingly unstructured gray bulk forming the brain ${ }^{2}$. However, owing to a number of recent technical innovations in the field of light microscopy, times could not have been better to tackle this problem. In the 1820s, the first achromatic microscopes had been invented by Giovanni Batista Amici, Joseph von Fraunhofer, and the Parisian physicist Selligue, to name a few, and were quickly improved in the following years. These new microscopes triggered a breakthrough in the understanding of living tissue and enabled Theodor Schwann and Rudolph Virchow to formulate modern cell theory, stating that the cell was the atomic unit of life. But while this theory was soon accepted for all different kinds of tissue, the central nervous system was still believed to be a diffuse network of interconnected tubes. The situation improved when the Italian histologist Camillo Golgi invented a staining technique in 1873 which he called "reazione nera". Opposed to the popular Nissl stain, this new method colored only a small fraction (1-5\%) of the neurons, but affected the entirety of the cell and allowed visualization of the complete structure of single cortical neurons for the first time $e^{3}$. By the end of the $19^{\text {th }}$ century, light microscopes which were optically comparable to today's standards had become commercially available and the invention of the microtome and the formaldehyde fixation method completed the toolbox needed to explore the brain at the microscale. Ramón y Cajal, who was known to carry his microscope along with him while visiting colleagues abroad [5], learned of Golgi's staining technique in 1888 and refined it to perfection. For more than 25 years the analysis of the microscopic structure of the nervous system became an obsession for Cajal and his famous drawings are still recognized for their anatomical accuracy today. Fig.1.1 shows the tiny protrusions that he depicted covering all of the processes of the neuron, with the exception of the axon, which is extending to the bottom of the figure. These tiny protrusions, called dendritic spines, are a topic of this thesis, and a good example of the lasting impact of Cajal's research on modern neuroscience.

Looking back, this was only one of many occasions in which advances in microscopy have enabled groundbreaking new insights about the brain. When Ramón y Cajal received the Nobel Prize for his achievements together with Golgi in 1907, the dispute

\footnotetext{
${ }^{1}$ Translation taken from [2]

${ }^{2}$ The mentioned volume by A. v. Gehuchten already contained Cajal's latest drawings, but little else on this topic

${ }^{3}$ The exact chemical mechanism of the Golgi staining is still not understood today [4]
} 


\section{Introduction}

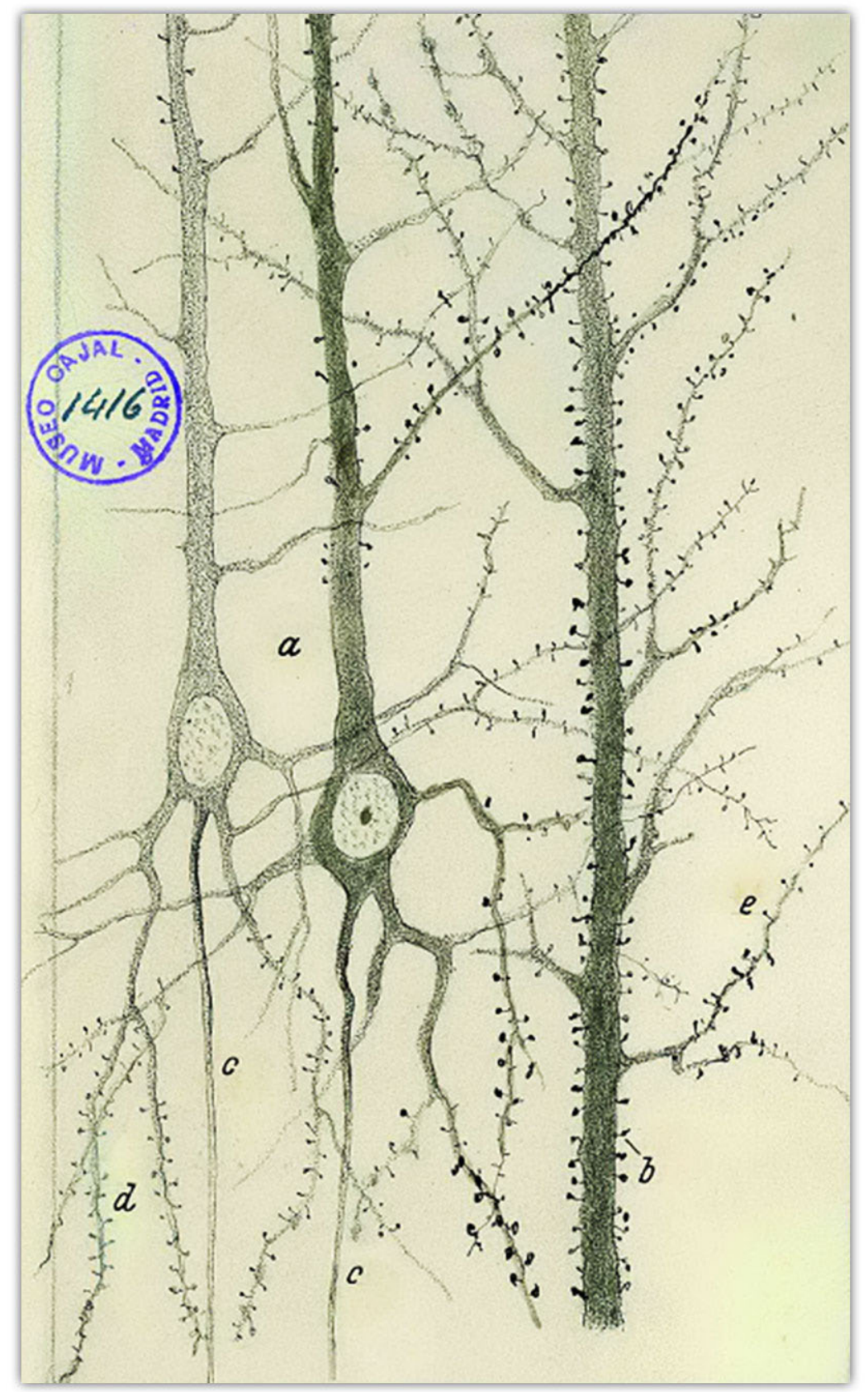

Fig. 1.1: An original drawing by Ramón y Cajal showing pyramidal cells of the rabbit cerebral cortex (1896, black ink and pencil). The dentritic spines are clearly depicted. Courtesy of Instituto Cajal (CSIC), Madrid. 


\section{Introduction}

about whether the neurons where physically connected at the synapses was still not settled. In fact, they even presented contrary conclusions to this question in their respective Nobel lectures [6]. But how could they have known? It took another half a century until 1954 when Palade and Palay were able to image the tiny gap between the cellular membranes at the synapse with an electron microscope [7]. The electron microscope has since then become the method of choice when imaging the smallest structures in the brain, for which the term "ultrastructure" was coined. However, the story of light microscopy does not end at this point. While a detailed knowledge of the cellular structure of the brain was the foundation for much of the research to follow, this knowledge alone provides only limited insight into how the brain actually works. While the brain's function is primarily determined by its dynamic behavior, sample preparation for electron microscopy inevitably turns any tissue into a lump of inanimate matter. Exploring the way that signals are processed by neurons and gaining insight on how their organization and interconnection changes in response to external stimulus therefore requires the use of a light microscope.

So how are neuroscientists using light microscopes today, a hundred years after the era of Cajal? In the beginning of the 1990s, the commercial availability of ultrafast solid-state lasers led to the development of the two-photon microscope [8]. Resulting from their superior capability to see through scattering tissue, the observation of neurons in the living mammalian organism became practical only shortly thereafter [9], rendering these microscopes indispensable tools in major neuroscientific laboratories. Possibly even more important, analogous to the Golgi staining, was the development of genetically encoded fluorescent proteins around the same time, which enabled highly specific image contrast at the molecular level in living organisms [10,11]. Since then, the desire to observe these fluorescent markers with higher speed and spatial resolution at any location in the intact brain tissue has driven the innovation in microscope development. The smallest microscopes presently weigh less than two grams and can be mounted on freely moving mice $[12,13]$; the fastest ones acquire up to a thousand images per second $[14,15]$; and again others give the ability to peek into the deepest layers of the cortex $[16,17]$.

While optical image resolution initially had to be sacrificed in order to maintain compatibility with living specimens, new microscopic methods based on light have been demonstrated during the last decade that approach the spatial resolution of electron microscopy [18]. These "super-resolution" methods have recently proven capable of delivering new insights to molecular processes in neurons [19-24], and are now increasingly being adopted by the scientific community. The question remains, however, if they can be applied to image cells directly in living higher animals which will be essential to fully understand how the neuron processes information in its natural, complex environment. The aim of this thesis is therefore to establish imaging at the nanoscale in the brain of a living mouse. After assessing some of the involved challenges using numerical tools, a powerful new microscope is implemented to image cellular structures in the brain with an unprecedented degree of spatial resolution. 


\subsection{At the frontier of optical microscopy}

When the optical manufacturer Carl Zeiss assigned the physicist Ernst Abbe in 1866 with the task of developing a new water immersion objective to challenge competitors, it was a right sensation in the field of optical instrumentation. Instead of designing the new optics by traditional means, which meant finding the right combinations of lens geometries and materials mainly by a lengthy trial-and-error approach, the idea was to calculate the right optical design based on nothing else but the laws of physics. In the following years, Abbe developed a solid theoretical framework accounting for the diffraction of light to tackle this problem and finally presented the requested objective in 1872. But there was a surprising insight resulting from his work which he published in the following year [25]. According to Abbe, the smallest focal spot that can be formed by a lens is fundamentally limited to ${ }^{1}$

$$
d \approx \frac{\lambda}{2 n \sin \alpha}=\frac{\lambda}{2 N A}
$$

The only parameters entering this relation are the wavelength $\lambda$ of the light being employed for the observation, the refractive index $n$ of the immersion medium between lens and sample and the semi-aperture angle $\alpha$ of the lens, which is usually expressed jointly with $n$ as the numerical aperture $N A=n \sin (\alpha)$. Since the refractive index cannot be increased beyond that of the lens material ( 1.5-1.7), the wavelength of the light seems to be the only parameter holding room for substantial improvement. However, UV light below $350 \mathrm{~nm}$ becomes increasingly difficult to handle as transparent materials for this wavelength range are scarce and high photon energies cause pronounced damage in biological samples. Therefore the simple statement in (1.1) implies that the resolution of any optical microscope is limited by physical principles to roughly about $200 \mathrm{~nm}$, no matter how well it is designed.

Despite this, light microscopy is still by far the most important imaging technology in the life sciences, which is mainly due to the development of the fluorescence microscope. While even its inventor Oskar Heimstädt [26] was not convinced of the usefulness of the new instrument initially ${ }^{2}$, it became a huge success after Albert Coons and his colleagues pioneered the immunostaining method in the beginning of the 40s [27]. This technique enabled high-contrast labeling of virtually any protein by chemically linking an antibody recognizing that protein to a fluorescent dye molecule. Presently, seven decades later, this protocol is still employed virtually unchanged in every field of modern biology.

Given the popularity of the light microscope, researchers have always tried to improve the imaging resolution beyond the stated diffraction limit. The first group of methods being successful in this regard avoided the issue by operating solely in the optical near-field of a light source, which implies distances of much less than the wavelength. One example is the scanning near-field optical microscope (SNOM), where a

\footnotetext{
${ }^{1}$ Measured as the full width half maximum (FWHM) of the intensity distribution

${ }^{2} \mathrm{He}$ concludes his paper with: "If and to what degree fluorescence microscopy will widen the possibilities of microscopic imaging only the future will show" [26]
} 


\section{Introduction}

sub-wavelength-sized aperture in a fine metal tip acts as the light source. This tip is then scanned across the surface at a very close distance to probe the optical properties in its vicinity [28]. The apertureless near-field microscopes on the other hand rely on the fact that the electric field component of an illuminating light source is greatly amplified close to the apex of a metal tip and interactions such as fluorescence excitation, second harmonic generation or surface-enhanced Raman scattering are therefore confined to a surrounding of only a few nanometers [29-31]. The last devices to be mentioned here are the so-called solid immersion lenses (SIL), which basically consist of a hemispherical solid with a high refractive index, such as gallium phosphide [32]. By filling the space between the primary immersion medium and the sample, the resolution is enhanced according to (1.1). However, as the refractive index of a biological sample is usually a lot lower than that of the SIL, the advantage is restricted to within the range of the decaying evanescent field of the totally reflected light, which is typically a fraction of the wavelength. SILs are therefore near-field optics for all practical matters even if the final detection is carried out in the far-field. A disadvantage common to all of these methods is their restriction to the sample surface which greatly restricts the range of problems they can address. Also, the involved experimental procedures are typically quite challenging. This explains why they never saw widespread use in the life sciences. The following section introduces methods which operate in the far-field, giving them a broad application range comparable to that of standard light microscopes.

\subsection{Far-field optical nanoscopy}

It has turned out that Abbe's law can be fundamentally broken in the case of fluorescence microscopy by exploiting photophysical properties of the fluorescent markers. When two fluorescing molecules are located in the sample less than $d(1.1)$ apart, they can neither be separately excited nor detected. This is expected since the excitation light source cannot be focused to a spot size smaller than the distance between the two and their diffraction spots will accordingly overlap on the detector to an extent that does not allow telling them apart. If, however, these molecules could be read out sequentially in time by making sure that at any given moment only one of them is able to contribute to the measured signal, the problem would be solved. STED microscopy ("STimulated Emission Depletion"), which was proposed in 1994 [33], was the first microscopy technique based on this principle. The underlying switching mechanism is stimulated emission [34], which is illustrated in the Jablonski diagram in Fig.1.2. A fluorescing molecule which absorbs a photon of the excitation laser undergoes a transition from the electronic ground state $S_{0}$ to the first excited state $S_{1}$ and usually remains in the excited state for an average time of a few nanoseconds before it spontaneously decays back to the ground state and thereby emits a red-shifted fluorescence photon. While residing in the excited state, however, this transition can also be resonantly induced by an external photon, leading to the emission of a photon with the same wavelength, phase and propagation direction. In STED microscopy, stimulated emission is induced by a second laser which is referred to as the STED- or depletion laser in the following. When the focus of this laser is overlaid with the focus of the 


\section{Introduction}

excitation laser and its wavelength is spectrally filtered from the detection path, the number of detected fluorescence photons is reduced. Increasing the intensity of the STED laser continuously decreases the fluorescence signal until it approaches zero and the fluorescing molecules are effectively switched off. In order to enhance imag-

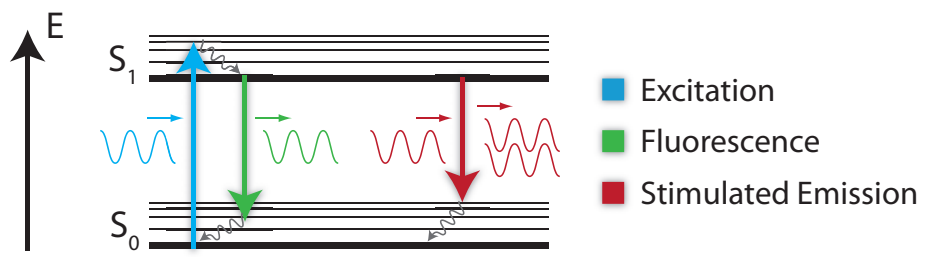

Fig. 1.2: Jablonski diagram showing the processes of fluorescence and stimulated emission, which is the switching principle underlying STED microscopy.

ing resolution with this effect, a special intensity distribution having a zero intensity at its center is chosen for the depletion laser. The so-called Gauss-Laguerre mode has proven to be a good choice, as it gives rise to a doughnut-like pattern in the focal plane (Fig. 1.3). When this $\mathrm{PSF}^{1}$ is now aligned with that of the excitation laser, it will switch off part of the fluorophores everywhere except at its center. In this way, the sample volume contributing to the detected signal is reduced and the effective resolution of the microscope is now given by [35]:

$$
d_{S T E D} \approx \frac{\lambda}{2 n \sin \alpha \sqrt{1+a I_{S T E D} / I_{S}}}=\frac{d}{\sqrt{1+a \zeta}}
$$

where $d$ is again the diffraction-limited resolution found in (1.1) and $I_{S T E D}$ denominates the peak intensity at the crest of the STED PSF. The saturation intensity $I_{S}$ is a spectroscopic parameter describing the depletion laser intensity at which half of the fluorescence of a given dye is suppressed at a certain STED wavelength. The dimensionless depletion factor $\zeta=I_{S T E D} / I_{S}$ is introduced for convenience and $a$ is a constant factor taking into account the geometry of the focal intensity distribution of the STED laser. As apparent from (1.2), there is no physical limit for the achievable resolution in STED microscopy. As long as the fluorophore is sufficiently photostable, $I_{S T E D}$ can be increased to yield molecular or even better resolution as demonstrated in [36]. While the presented doughnut-shaped PSF enhances the resolution only in the lateral direction, 3D enhancement can be achieved by applying a different depletion pattern [37]. As opposed to every other existing super-resolution method, STED microscopy was shown to work with regular fluorescent proteins [38], which allowed the use of existing transgenic mouse models in the measurements presented later on. Further, it was proposed that the approach outlined above can be generalized to all light-emitting markers that are reversibly switchable between a bright and a dark state by means of light. The general concept is called RESOLFT microscopy, with the acronym RESOLFT standing for "Saturable OpticaL Fluorescence Transition". Apart from STED, depleting the

\footnotetext{
${ }^{1}$ The point spread function (PSF) of an imaging system describes its response to a point source or point object. Correspondingly, a diffraction-limited focus in the sample plane is the PSF of a point source in the image plane. The term PSF is therefore used synonymously for the focal intensity distribution here.
} 


\section{Introduction}
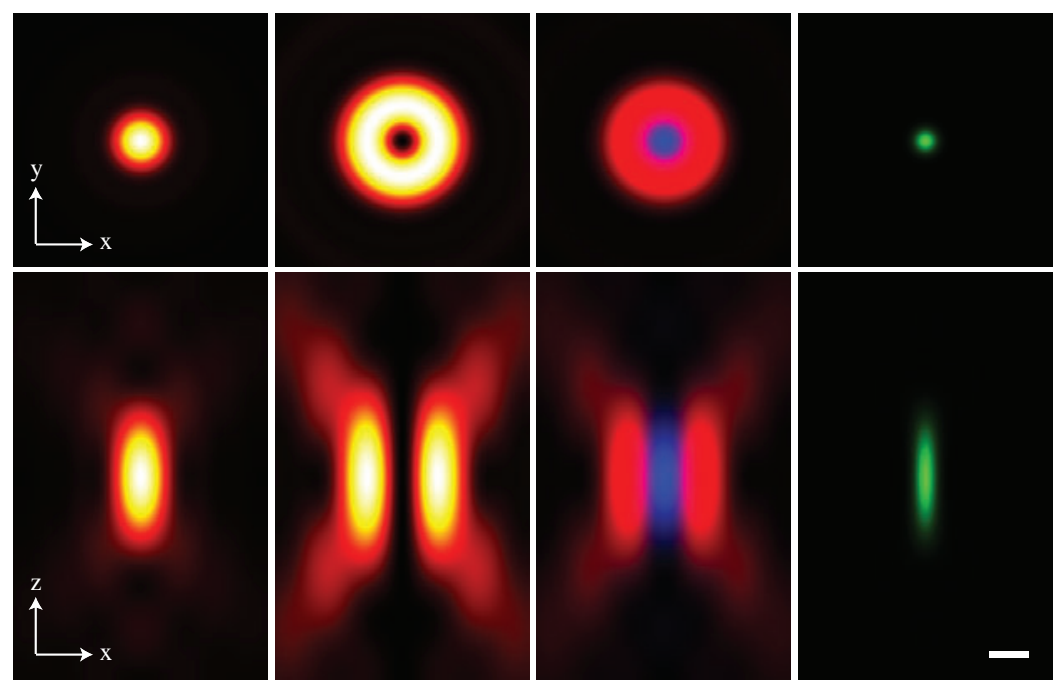

Fig. 1.3: Point spread functions of a STED microscope with a doughnut-shaped depletion pattern. From left to right: excitation laser, STED laser, spatial overlay of STED and excitation lasers and the effective STED PSF at $\varsigma=20$

ground state has proven successful $[39,40]$ as well as using photo-switchable compounds that can be reversibly switched with UV light between isomers with different fluorescence properties $[41,42]$. The latter could recently be demonstrated in living specimen, benefiting from the development of reversibly switchable fluorescent proteins (RSFPs) with enhanced photostability [43, 44].

While photo-emitters in the sample are switched at precisely defined positions in RESOLFT microscopy, this so-called targeted switching is not inherent to the concept of beating the diffraction limit by time-sequential readout. A second class of methods is based on using photo-switchable markers which are imaged onto a camera in a widefield configuration. By applying a low amount of activation light, a subset of these markers is randomly activated such that on average there is only a single molecule active in any diffraction-limited area of the image at any given time. After a short active period, the markers are switched off again and the process is repeated. Every molecule can now be localized by calculating the center-of-mass of its diffraction spot on the camera with a precision depending only on the number of collected photons per molecule and cycle. By plotting the coordinates obtained in this way from all switching cycles, an image with sub-diffraction resolution of the distribution of the molecules can be reconstructed. The methods belonging to this group, such as PALM [45], STORM [46], GSDIM [47] and many others, differ mainly in the employed switching mechanism. By breaking the focal plane symmetry of the PSF, the resolution can readily be enhanced in 3D $[48,49]$.

Although the implementation of super-resolution methods based on stochastic switching is technically less challenging compared to methods based on targeted switching, this comes at the cost of a few drawbacks. First, each of the methods is restricted to a few uncommon labeling systems (PALM, STORM) or requires at least a special chemical environment (GSDIM), which limits their application in vivo. Also, current camera 
frame rates and marker brightness limit the achievable speed to the range of minutes per reconstructed frame, resulting in image artifacts if too much motion is present in the sample. Apart from this, RESOLFT-type microscopy has the charm of not requiring numerical processing of the recorded data - the image is formed by plotting a series of sequential point measurements, making noise and motion artifacts easy to identify by visual inspection. 


\section{STED microscopy through planar dielectric interfaces}

In all far-field light microscopes, the light which is propagating from the front aperture of the objective lens traverses the immersion medium and eventually a cover slip before entering the actual sample volume. Under ideal optical conditions, as found in the case of properly employed oil-immersion objectives, the refractive indices of all three media are matched in order to avoid refraction and Fresnel-losses at the dielectric interfaces separating them. In general applications though, this is often not feasible as the refractive index of the sample cannot be influenced and the wave front is therefore inevitably disturbed on its path to the focal point. This is especially true if a living sample is to be studied and replacing the contained water by an index-matched mounting medium is therefore not an option. This means that imaging today's most interesting subjects such as living cells, cultured tissue samples and, ultimately, whole intact organisms will not attain its full potential without taking the optical properties of these predominantly aqueous samples into account. As STED microscopy has recently become popular for diffraction-unlimited imaging also in living specimen, the question arises to which extent its performance is influenced by the dielectric interfaces in the sample space.

Effects of refractive index mismatch between the immersion and the sample medium have been extensively treated for confocal and multi-photon microscopes [50-57]. As a consequence, some microscope objectives have been equipped with mechanisms to compensate for these aberrations and now enable diffraction-limited performance for a wide range of samples when adjusted carefully. However, those findings cannot be directly transferred to the case of STED microscopy, where in addition to the excitation and detection pathways also the STED beam has to be accounted for. This beam could potentially be more sensitive to aberrations as it usually carries a complex phase pattern to create a PSF featuring a point of zero intensity. Due to the highly saturated stimulated emission process which it induces over most of the PSF volume, it is not immediately obvious how small changes of the focal intensity distribution influence the effective imaging properties. This is especially true for the zero intensity spot of the pattern, where it will be shown that even small parasitic intensities severely compromise the attainable spatial resolution and signal-to-noise ratio.

The following chapter will assess the influence of index-mismatch on image formation in STED microscopy by means of numerical simulations. Compared to previous work on this topic [58-60], the aim is to go beyond a mere phenomenological description by quantifying the effects under the concrete conditions found when imaging intact brain tissue. The performance of different objectives is compared not only regarding their best-case performance but also with respect to robustness towards deviations from ideal conditions which are likely to be present during experiments. 


\subsection{Vectorial simulation of focal fields}

The focal intensity distributions of the involved laser beams are numerically derived from the corresponding complex vector fields $\mathbf{A}^{(\mathbf{0})}$ at back aperture of the objective lens. Apart from $\mathbf{A}^{(\mathbf{0})}$, which is also termed the vectorial pupil function in the following, the wavelength $\lambda$, the semi-aperture angle $\alpha$ and the refractive indices $n_{1}$ and $n_{2}$ of the immersion medium characterize the optical system. To start with, it is assumed that $n_{1}$ and $n_{2}$ are equal, meaning that the refractive indices of the immersion medium and the sample are perfectly matched. An effective method to accurately calculate the vec-

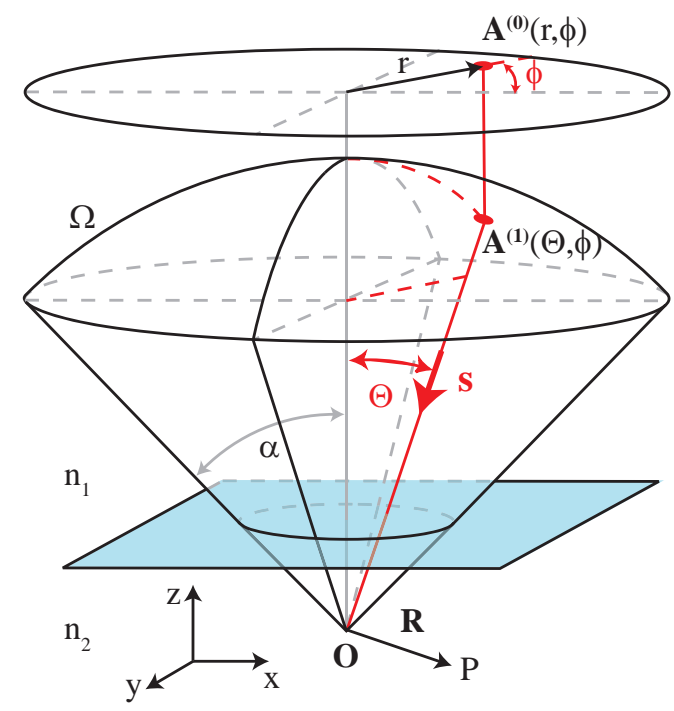

Fig. 2.1: Coordinate system used for the evaluation of the diffraction integral. The spherical wave cap $\Omega$ centered about origin $\mathbf{O}$ is decomposed into a spectrum of plane waves propagating at different angles $\Theta$ relative to the optical axis $\mathrm{z}$. $\mathbf{R}$ is a vector to the observation point $\mathrm{P}$ close to the origin. The complex vector field $\mathbf{A}^{(\mathbf{0})}$ entering into the back aperture of the objective is transformed to the field $\mathbf{A}^{(\mathbf{1})}$ on $\Omega$ after refraction by the lens. In the depicted case of matched refractive indices $n_{1}$ and $n_{2}$, the spherical wave emerging from the microscope objective propagates undisturbed to the geometrical focal point.

tor field $\mathbf{E}$ near the focal point $\mathrm{O}$ of a high-NA microscope objective was developed by Richardson \& Wolff in 1959 [61]. The geometry of the problem and the corresponding angles and vectors are shown in Fig. 2.1. Using an approximation proposed by Debye [62], the field is represented as a superposition of plane waves propagating in different directions $\mathbf{s}$, which are originating from a spherical cap with radius $f$ exiting the front aperture of the objective. The electromagnetic field at a point $\mathrm{P}$ with the cartesian coordinates $r_{p} \cdot\left\{\sin \Theta_{p} \cos \phi_{p}, \sin \Theta_{p} \sin \phi_{p}, \cos \Theta_{p}\right\}$ is retrieved by integrating the complex vector field $\mathbf{A}^{(\mathbf{1})}$ on $\Omega$ delimited by a cone covering the aperture angle: 


$$
\begin{aligned}
\mathbf{E}^{(\mathbf{1})}(P) & =\frac{i f}{\lambda} \int_{0}^{\alpha} \int_{0}^{2 \pi} \mathbf{A}^{(\mathbf{1})}(\Theta, \phi) \exp \{-i \mathbf{s} \cdot \mathbf{R}\} \sin \Theta d \Theta d \phi \\
& =\frac{i f}{\lambda} \int_{0}^{\alpha} \int_{0}^{2 \pi} \mathbf{A}^{(\mathbf{1})}(\Theta, \phi) \exp \left\{-i k r_{p} \kappa^{(1)}\right\} \sin \Theta d \Theta d \phi
\end{aligned}
$$

with the phase factor $\kappa^{(1)}$ being

$$
\kappa^{(1)}=n \cdot\left[\sin \Theta \sin \Theta_{p} \cos \left(\phi-\phi_{p}\right)+\cos \Theta \cos \Theta_{p}\right]
$$

and the apodization introduced by an aplanatic lens

$$
\begin{aligned}
& \mathbf{A}^{(1)}=\sqrt{\cos \Theta} \cdot\left[A_{x}^{(0)}\left(\begin{array}{c}
\cos \Theta+(1-\cos \Theta) \sin ^{2} \phi \\
(\cos \theta-1) \sin \phi \cos \phi \\
\sin \Theta \cos \phi
\end{array}\right)\right. \\
& \left.+A_{y}^{(0)}\left(\begin{array}{c}
(\cos \theta-1) \sin \phi \cos \phi \\
\cos \Theta+(1-\cos \Theta) \cos ^{2} \phi \\
\sin \Theta \sin \phi
\end{array}\right)\right]
\end{aligned}
$$

In order to calculate the effective PSF of a STED microscope, (2.1) is first evaluated independently for the excitation, the confocal detection and the depletion PSF. Then, the fluorescence at every sampling point is reduced by the suppression factor $\eta$ derived from the intensity of the depletion PSF at that point and a given saturation factor $\varsigma$, followed by the convolution of the result with the confocal detection PSF. In this way, effective resolution and signal levels in STED microscopy have been predicted in excellent agreement with experimental observations [35]. In the following sections, (2.1) will be extended to account for different aberrations arising from dielectric interfaces in the focal pathway.

\subsection{Aberrations introduced by a single dielectric interface}

In the past, when living specimen were to be observed directly within an aqueous buffer medium, this was usually achieved with an inverted STED microscope and a standard oil-immersion objective[38, 63, 64]. In this configuration, the immersion oil and the glass bottom of the sample chamber form an optically uniform layer that extends to the interface with the buffer medium. Despite the severe refractive index mismatch between the media, the results obtained from these experiments are usually very satisfying as long as the features of interest are located in direct vicinity of the glass-water interface or at most a few microns away from it. In order to find the limitations of this simple approach when imaging brain tissue, the optical effects of the interface on the performance of a STED microscope are quantified here for different depletion PSFs. 


\subsubsection{Enhanced numerical model}

To investigate this case, (2.1) is extended as proposed by Török et al. in 1995 [55]. Note that this solution was chosen over the equivalent one proposed earlier by Hell et al. [57, 65] solely due to computational efficiency considerations.

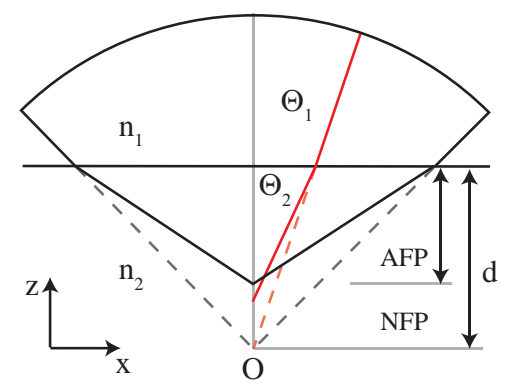

Fig. 2.2: In the case of $n_{1}>n_{2}$, aberrations occur and the focal point is shifted towards the dielectric interface.

The extended geometry of the problem can be seen in Fig. 2.2. Plane waves propagating at an angle $\Theta_{1}$ with respect to the $\mathrm{z}$ axis are refracted at the interface at position $-d$ and continue at $\Theta_{2}$ according to Snell's law: $\sin \Theta_{2}=n_{1} / n_{2} \sin \Theta_{1}$. The initial wave spectrum will therefore undergo changes in phase and amplitude, breaking the focal plane symmetry and leading to an elongation of the PSF along the optical axis. Also, part of the light is reflected according to Fresnel's equations. As field components oriented perpendicularly with respect to the interface will experience different amplitude changes compared to the ones oriented in parallel, each of the polarization states of the initial pupil function will experience a different effective apodization. Furthermore, it should be noticed that the focal point, defined as the point of maximum intensity, is shifted away from the origin. Translating the objective lens relative to the sample along the optical axis will thus not shift the position of the focal plane in the sample by the same distance. For the sake of clarity, the nominal focus position (NFP) is therefore distinguished from the actual focus position (AFP) in the following.

For a single dielectric interface the extended diffraction integral is

$$
\mathbf{E}^{(\mathbf{2})}(P)=\frac{i f n_{1}}{\lambda} \int_{0}^{\alpha} \int_{0}^{2 \pi} \mathbf{A}^{(\mathbf{2})}\left(\Theta_{1}, \phi\right) \exp \left\{-i k\left[r_{p} \kappa^{(2)}+\Psi^{(2)}\left(\Theta_{1}, d\right)\right]\right\} \sin \Theta_{1} d \Theta_{1} d \phi
$$

with the phase factors $\kappa^{(2)}$ and $\Psi^{(2)}$ defined as

$$
\begin{aligned}
\kappa^{(2)} & =n_{1} \sin \Theta_{1} \sin \Theta_{p} \cos \left(\phi-\phi_{p}\right)+n_{2} \cos \Theta_{2} \cos \Theta_{p} \\
\Psi^{(2)}\left(\Theta_{1}, d\right) & =d\left(n_{2} \cos \Theta_{2}-n_{1} \cos \Theta_{1}\right)
\end{aligned}
$$

The aberration function $\Psi^{(2)}(2.6)$ obviously describes spherical aberration as it only 


\section{STED microscopy through planar dielectric interfaces}

depends on the polar angle $\Theta_{1}$. Note that $\Theta_{2}$ is related to $\Theta_{1}$ by Snell's law, as mentioned before, and does therefore not represent an independent variable. The phase factor $\kappa^{(2)}(2.5)$, on the other hand, describes the same phase relation in the new sample medium as $\kappa^{(1)}(2.2)$ did in the initial case and therefore does not introduce any aberration by itself. The complex field distribution $\mathbf{A}^{(2)}$, which enhances $\mathbf{A}^{(1)}$ (2.3) to account for the apodization and phase shift introduced by the dielectric interface, is generalized from [55] to handle arbitrary transverse polarization states in the pupil function $\mathbf{A}^{(0)}$. In cartesian coordinates one gets

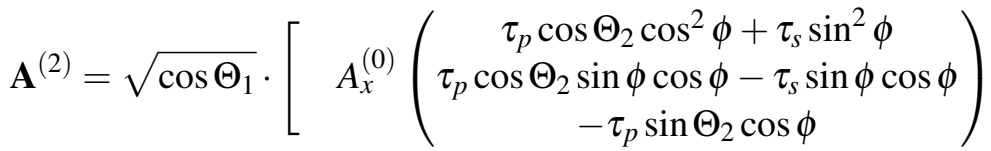

$$
\begin{aligned}
& \left.+A_{y}^{(0)}\left(\begin{array}{c}
\tau_{p} \cos \Theta_{2} \sin \phi \cos \phi-\tau_{s} \sin \phi \cos \phi \\
\tau_{p} \cos \Theta_{2} \sin ^{2} \phi+\tau_{s} \cos ^{2} \phi \\
-\tau_{p} \sin \Theta_{2} \sin \phi
\end{array}\right)\right]
\end{aligned}
$$

with the Fresnel coefficients $\tau_{p}$ and $\tau_{s}$ for parallel and perpendicular field components defined as

$$
\tau_{s}=\frac{2 \sin \Theta_{2} \cos \Theta_{1}}{\sin \left(\Theta_{1}+\Theta_{2}\right)} \quad \tau_{p}=\frac{2 \sin \Theta_{2} \cos \Theta_{1}}{\sin \left(\Theta_{1}+\Theta_{2}\right) \cos \left(\Theta_{1}-\Theta_{2}\right)}
$$

For $n 1>n 2$, the case of total internal reflection (TIR) has to be treated where $\Theta_{1}$ is larger than the critical angle $\Theta_{C}=\arcsin \left(n_{2} / n_{1}\right)$. As the evanescent field at the interface is not in the scope of this work, the integration over $\Theta_{1}$ can simply limited to $\Theta_{C}$ instead of $\alpha$. For the numerical integration of (2.4), an existing program written in the MATLAB environment (The Mathworks, Natick/MA, USA) [66] originally developed for (2.1) was extended. All calculations were performed on a standard desktop PC.

\subsubsection{A word on the refractive index of brain tissue}

As the discussion in this chapter is centered around the optical conditions found when imaging in the living brain, probably the most important parameter for all of the numerical modelling to follow is the average refractive index of brain tissue. Despite the importance of this value for all studies of the brain by means of light, there is not an established reference value or a standard procedure to determine it yet. While quantitative phase microscopy in the visible suggests a value around $n=1.371$ [67], a number as high as $n=1.395$ [68] was derived from computer tomography (CT), representing the upper bound of values published so far. It is also unclear whether the refractive index varies between the intact brain in vivo and fresh cut samples which have been used for most studies. In a recent work, the refractive index was therefore measured in-vivo for the first time using optical coherence tomography (OCT) with near-infrared (NIR) light [69], yielding a relatively low value of $n=1.353$ compared to other OCT experiments showing a refractive index of $n=1.37$ [70]. It should be mentioned though, that the authors expect significantly higher values $(+0.01)$ for visible light. Finally, a simple measurement was performed for comparison using a calibrated digital refractometer 
(DSR $\lambda$, Schmidt+Haensch, Berlin) which derives the refractive index from measurements of the critical angle. A series of fresh samples from bovine and mouse brain was analysed at $36^{\circ} \mathrm{C}$, yielding an average value of $n=1.374$ with a low variance $( \pm 0.005)$. The refractive index which was finally chosen for all of the following numerical studies is $n=1.37$, which is approximately the median of the presented measurements based on visible or NIR light. In fact, the results obtained from the simulations in this chapter do not change significantly for typical variations of the refractive index ${ }^{1}$. This is important to realize, as such variations are likely to be present in the studied samples later on, depending on the imaged brain region and probably also depending on the individual subject.

\subsubsection{D STED microscopy in the brain with oil-immersion objectives}

Currently, in most implementations of STED microscopes, a NA1.4 oil-immersion objective is used together with a doughnut-shaped PSF to laterally enhance the resolution of a confocal laser-scanning microscope (CLSM). The first simulation series is therefore dealing with this popular setting. The results which are presented in the following do not qualitatively depend on the chosen wavelengths, so they are either expressed in units of the excitation wavelength or normalized to the initial value of a data series. All depicted PSFs, however, were calculated for excitation and STED wavelengths of $488 \mathrm{~nm}$ and $590 \mathrm{~nm}$, respectively. These lasers lines are currently being used for STED microscopy of fluorescent proteins. The depletion factor $\varsigma=70$ was chosen as to result in an effective lateral resolution of about one tenth of the excitation wavelength, which is typically achieved when working with fluorescent proteins in living specimen. Furthermore, a confocal detection pinhole with a diameter of one Airy disc was assumed for all of the following simulations. Note that the size of the pinhole has only very little effect on the resolution in STED microscopy for large $\varsigma$.

The numerical results in Fig. 2.3A clearly show the drastic deterioration of imaging performance when the focal plane is moved into the sample. The fluorescence signal is thereby defined throughout this chapter as the detected peak value, corresponding to the maximum fluorescence intensity of a single molecule in the focus. Note that due to the broken focal plane symmetry, there are different ways to define the resolution here. The upper dashed line in the graph is obtained when measuring the FWHM across the brightest point of the effective PSF, which is the intuitive way to define the lateral resolution. However, when looking for the narrowest cross-section, the minimum is found at just a few percent lower brightness slightly above this nominal focal point because of the closer spacing of the lobes of the STED PSF. The lower dashed line corresponds to this second metric, which can be defining for objects having very small axial extension such as nano-sized beads.

At an NFP of only $5 \mu \mathrm{m}$ the FWHM of the effective PSF has broadened to $150 \%$ and the detected peak fluorescence signal has dropped to half of its initial value. The drop

\footnotetext{
${ }^{1}$ The average refractive index of any soft tissue is usually determined by the relative ratio of cytosol(1.35) to fatty lipids, as found in lipid membranes (1.46) [71]. Considering that the variation of lipid content in a single tissue type as the gray matter in the brain is expected to be rather low, the local refractive index should usually lie within \pm 0.01 of the average value
} 

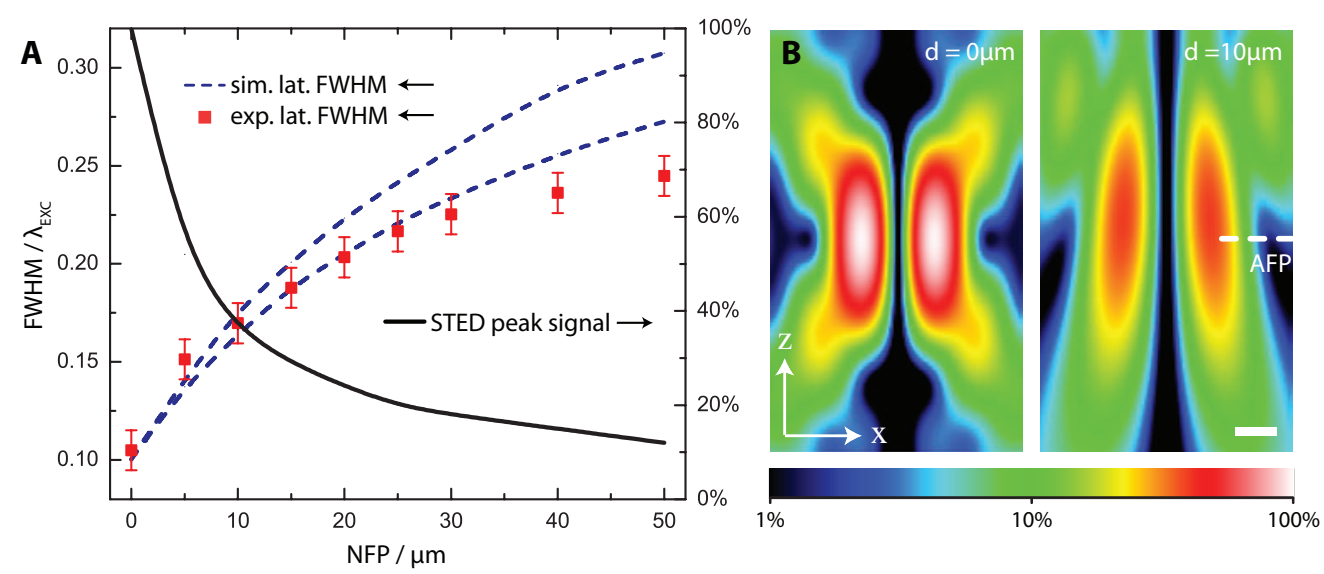

Fig. 2.3: When using an oil-immersion objective with NA1.4 for STED microscopy inside brain tissue, the resolution and the peak fluorescence signal decrease rapidly with increasing imaging depth, as shown for $\varsigma=70$ in (A). While spherical aberration affects symmetry, axial extension and peak intensity of the applied STED PSF, the central intensity always remains zero (B). The two lines being depicted for the simulated FWHM in (A) correspond to the result at the NFP (upper) and the minimum FWHM, which is found above the focus (lower). Experimental values from Sec. 2.2.4 are included for comparison. Scale bar $=200 \mathrm{~nm}$.

in resolution is thus more pronounced than in a regular CLSM ${ }^{1}$ which is due to the depletion PSF not only being spatially broadened but also experiencing a rapidly declining peak intensity. The loss of signal on the other hand, is not a consequence of the STED configuration but already present in the confocal system. As can be seen in the examples depicted in Fig. 2.3B, the central zero intensity of the STED PSF is conserved at all depths, and therefore fluorescence in this spot remains unaffected. The resolution of the STED microscope is better than the diffraction limit over the whole depth range. This stability is obviously owed to the fact that the vortex phase pattern leading to this PSF does not have a radial dependence and therefore a radially symmetric aberration will not affect the relative phase at the focal point or anywhere else on the optical axis. Nevertheless, it is not advisable to use an oil-immersion objective in a setting like this anywhere but in immediate vicinity of the coverslip due to the inefficient use of inhibition and fluorescence light.

\subsubsection{Experimental validation}

In order to confirm the findings of the numerical model, 40nm fluorescent polymer beads (Yellow-green FluoSpheres ${ }^{\circledR}$, Invitrogen, Carlsbad/CA) were measured with identical parameters on a custom-built STED microscope similar to the one presented in [72]. The beads were embedded in glycerol gelatin with a refractive index of $n=1.37$ 2 and mounted on a molded microscope slide sealed with a cover slip to form a volu-

\footnotetext{
${ }^{1}$ The confocal FWHM broadens from $193 \mathrm{~nm}$ to $250 \mathrm{~nm}$ over the same range. Values are not included in Fig. 2.3 for better presentation.

${ }^{2} 0.9 \mathrm{~g}$ of bovine gelatin dissolved in a 21:79 mixture of glycerol and water (by weight fraction)
} 


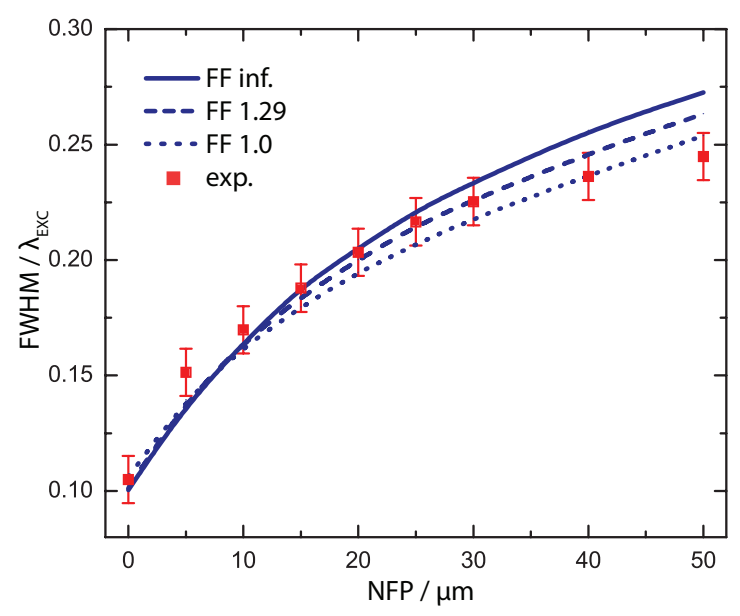

Fig. 2.4: Simulated resolution for different intensity distributions of the STED laser in the back aperture. When the fill factor is decreased, the sensitivity to refractive index mismatch is slightly reduced at nominal focal positions exceeding $10 \mu \mathrm{m}$. The STED power in the objective aperture was adjusted to obtain equal peak intensity for all STED PSFs.

metric test sample. A continuous-wave (CW) fiber laser with a wavelength of $590 \mathrm{~nm}$ (VFL-P-1000-590, MPB Communications, Montreal) was used for depletion, which was already taken into account in the simulations above. In order to compare to the previous results, the STED power at the back aperture of the objective was set to $300 \mathrm{~mW}$, yielding an initial resolution of about 50nm. The FWHM of the beads in the STED images at different NFPs was then determined algorithmically with an image analysis tool and averaged. In order to retrieve the optical resolution from the measured bead diameters, the finite size of the polymer beads has to be compensated for, as presented in [73]. As only the smallest 5\%- fraction of the beads was included for analysis, it makes sense to compare the measurements with the minimum predicted FWHM in Fig. 2.3A, represented by the lower dashed curve. In order to validate to upper line corresponding to the FHWM in the focal plane, one would have to select the brightest beads instead. This, however, turned out to be impractical as the brightest signal in the recorded images is usually stems from bead clusters, which cannot be reliably excluded in 2D analysis due to the unknown focal position. Up to an NFP of $30 \mu \mathrm{m}$, the measurements are in excellent agreement with the numerical results, whereas afterwards there seems to be a systematic under-estimation of the achievable resolution. This could be explained by the fact that the pupil function was modeled based on a uniform intensity distribution in the pupil plane, whereas in the experiment a Gaussian beam with a limited fill factor is used ${ }^{1}$.

The effect of different fill factors (FF) is shown in Fig. 2.4, with 1.29 corresponding to the setting in the experiment. At lower FF the sensitivity to refractive index mismatch is reduced. However, even if the fit to the experimental data could be visibly improved for large NFPs, the progression for the different settings is very similar in the relevant range up to $10 \mu \mathrm{m}$ where imaging is still feasible. For this reason, and in order to keep

\footnotetext{
${ }^{1}$ The fill factor describes the ratio of the Gaussian beam diameter to the physical back aperture of the microscope objective. The beam diameter is measured at the $1 / e^{2}$ intensity level
} 
the parameter space manageable, a uniform intensity distribution of the STED laser in the back aperture is assumed for all of the following simulations (corresponding to an infinite FF).

\subsubsection{D STED with an oil-immersion objective}

While the STED PSF resulting from a vortex phase pattern can be used in an indexmismatched setting to a limited extent, it has turned out to be impractical to get acceptable results when using a PSF that mainly increases the resolution along the optical axis in such a case. The best phase pattern to create such a PSF was among the first to be adopted for STED microscopy [37] and consists of a central circular area with a phase retardation of $\pi$ which is accounting for half of the aperture area.

Looking at the results in Fig. 2.5A, it is immediately obvious that this depletion PSF is much more susceptible to spherical aberrations as compared to the doughnut-shaped one. Considering only the relative broadening of the FWHM in the axial and the lateral direction, the 3D PSF does not perform significantly worse. However, the crucial point here is that the signal level drops much faster and, more importantly, immediately drops below the signal that would be found without the depletion PSF. The reason for this is that the zero-intensity is not conserved at the central minimum, and the signal is therefore quenched by the STED laser. Resolution improvement in three dimensions can thus only be achieved when correcting for aberrations in the system, which explains the inability so far to get 3D super-resolved images of brain slices with oil-immersion objectives.

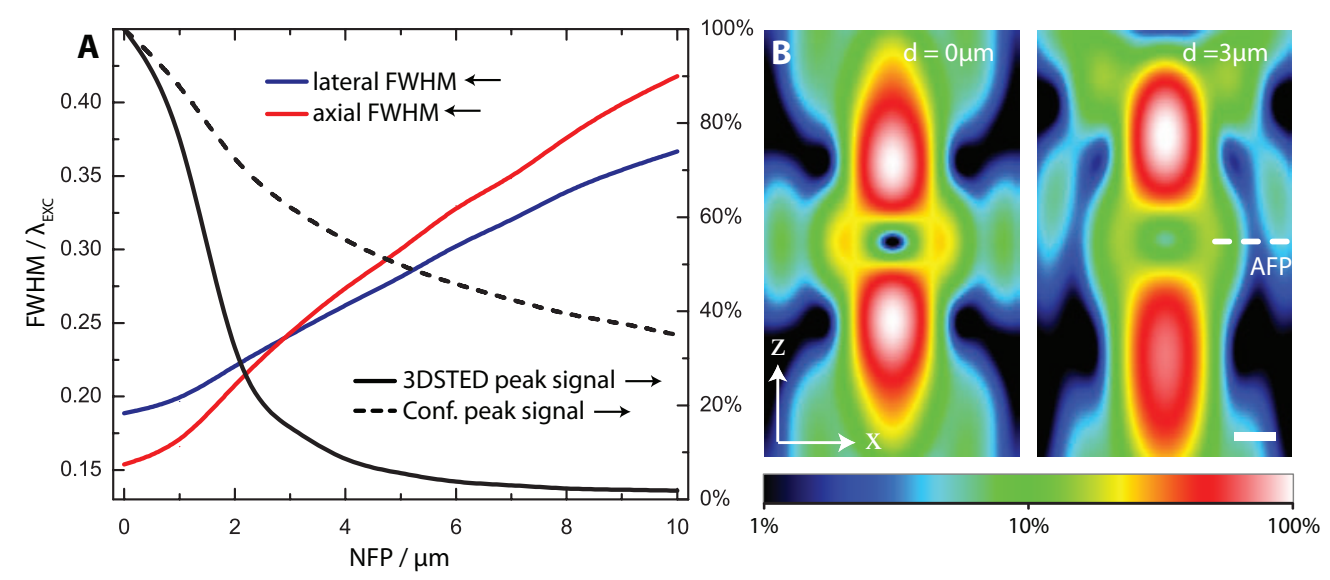

Fig. 2.5: When using an oil-immersion objective in the 3D-STED configuration to image inside brain tissue, the signal decreases much faster than in the confocal counterpart (A). This is caused by the non-zero intensity which is accumulating at the central minimum of the depletion PSF due to spherical aberrations (B). Simulations were performed for the same STED peak intensity as in the 2D case $(\varsigma=70)$. Scale bar $=$ $200 \mathrm{~nm}$. 


\subsection{Adding a glass coverslip to the model system}

In order to handle situations where more than a single dielectric interface is present, a generalization to (2.4) is available for general stratified media [74]. Although this framework can treat any number of layers with different refractive indices, only one more interface has to be introduced here, which is enough to describe a glass coverslip with adjacent heterogeneous media.

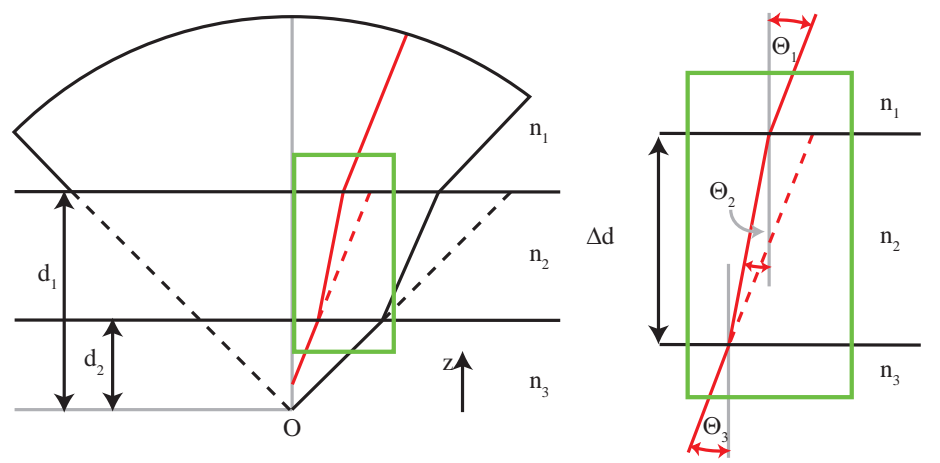

Fig. 2.6: An additional interface is introduced to account for a glass coverslip with a thickness of $\Delta d$ and a refractive index $n_{2}$, where $n_{1}, n_{3}<n_{2}$.

The diffraction integral for three media can be written in the same form as (2.4):

$$
\mathbf{E}^{(\mathbf{3})}(P)=\frac{i f n_{1}}{\lambda} \int_{0}^{\alpha} \int_{0}^{2 \pi} \mathbf{A}^{(\mathbf{3})}\left(\Theta_{1}, \phi\right) \exp \left\{-i k\left[r_{p} \kappa^{(3)}+\Psi^{(3)}\left(\Theta_{1}, d\right)\right]\right\} \sin \Theta_{1} d \Theta_{1} d \phi
$$

with the modified phase coefficients

$$
\begin{aligned}
\kappa^{(3)} & =n_{1} \sin \Theta_{1} \sin \Theta_{p} \cos \left(\phi-\phi_{p}\right)+n_{3} \cos \Theta_{3} \cos \Theta_{p} \\
\Psi^{(3)}\left(\Theta_{1}, d\right) & =d_{2} n_{3} \cos \Theta_{3}-d_{1} n_{1} \cos \Theta_{1}
\end{aligned}
$$

While $\kappa$ is effectively identical to the previous case, the term $\Psi$ needs to be further discussed, as the complex field $\mathbf{A}^{(\mathbf{3})}$ :

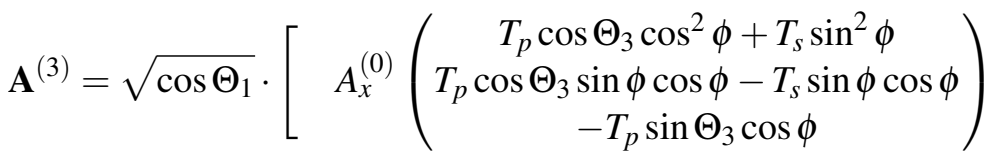

$$
\begin{aligned}
& \left.+A_{y}^{(0)}\left(\begin{array}{c}
T_{p} \cos \Theta_{3} \sin \phi \cos \phi-T_{s} \sin \phi \cos \phi \\
T_{p} \cos \Theta_{3} \sin ^{2} \phi+T_{s} \cos ^{2} \phi \\
-T_{p} \sin \Theta_{3} \sin \phi
\end{array}\right)\right]
\end{aligned}
$$

now contributes an additional phase term introduced by the transmission coefficients 


$$
T_{s, p}=\frac{t_{s, p}^{(1)} t_{s, p}^{(2)} \exp \left(i k_{2} \Delta d \cos \Theta_{2}\right)}{1+r_{s, p}^{(1)} r_{s, p}^{(2)} \exp \left(2 i k_{2} \Delta d \cos \Theta_{2}\right)}
$$

based on the amplitude coefficients at the two interfaces

$$
\begin{aligned}
t_{s}^{(j)} & =\frac{2 n_{j} \cos \Theta_{j}}{n_{j} \cos \Theta_{j}+n_{j+1} \cos \Theta_{j+1}} & r_{s}^{(j)} & =\frac{2 n_{j} \cos \Theta_{j}-n_{j+1} \cos \Theta_{j+1}}{2 n_{j} \cos \Theta_{j}+n_{j+1} \cos \Theta_{j+1}} \\
t_{p}^{(j)} & =\frac{2 n_{j} \cos \Theta_{j}}{n_{j+1} \cos \Theta_{j}+n_{j} \cos \Theta_{j+1}} & r_{p}^{(j)} & =\frac{2 n_{j+1} \cos \Theta_{j}-n_{j} \cos \Theta_{j+1}}{2 n_{j+1} \cos \Theta_{j}+n_{j} \cos \Theta_{j+1}}
\end{aligned}
$$

Note that for the cases which are to be considered here, it is possible to simplify (2.13). In a typical setup, $n_{1}$ and $n_{3}$ will be situated between the refractive index of water (1.33) and that of the glass coverslip (about 1.51), which always forms the middle layer. In this case, the product $r_{s, p}^{(1)} r_{s, p}^{(2)}$ is much smaller than unity, and therefore the denominator of (2.13) can be omitted. The total aberration can then be written as

$$
\begin{aligned}
\Psi_{t o t} & =\Psi^{(3)}+d_{1} n_{2} \cos \Theta_{2}-d_{2} n_{2} \cos \Theta_{2} \\
& =d_{2}\left(n_{3} \cos \Theta_{3}-n_{1} \cos \Theta_{1}\right)+\Delta d\left(n_{2} \cos \Theta_{2}-n_{1} \cos \Theta_{1}\right)
\end{aligned}
$$

This in turn means that there are effectively two decoupled spherical aberration terms introduced by the coverslip and the sample layer, respectively. Both of these will have to be corrected for independently in the microscope objective. Due to technical considerations, it is likely that these corrections are implemented in the simplified form (2.16). Regarding the total aberration, it is interesting to note that the penetration depth $d_{2}$ and the refractive index $n_{3}$ of the sample in principle cannot be perfectly corrected for with the single degree of freedom provided by the correction collar. This holds especially true for the marginal rays at high numerical apertures, which means that the manufacturer has to make some assumption about the sample and the depth range when designing the optics which are actuated by the correction collar. Regarding the coverslip, the microscope objective is usually corrected for a defined $(\# 1.5,170 \mu \mathrm{m})$ thickness. As the dependence of the coverslip aberration on $\Theta_{1}$ in (2.16) differs from that of the sample-induced term, the objective should be strictly used at that design thickness instead of correcting for a deferring coverslip thickness with the correction collar. This is especially true in cases where $n_{1}$ a $n_{3}$ differ largely.

The following calculations of the focal light intensity distributions were performed with the exact form of $T_{s, p}$ (2.13) in order to assess the influence of its small nonrotational-symmetric variations on the zero-intensity spot of the STED PSFs. Objective corrections where simulated by applying the inverted aberration terms according to (2.16). The apodization introduced by the angle-dependent transmission properties of the interfaces are thereby not affected. One has to keep in mind though, that the subtle details of the correction mechanisms are known only to the manufacturers. The presented calculations assume perfect correction mechanisms and therefore present a best-case study with respect to real-world experimental conditions. 


\subsubsection{Corrected objectives with glycerol and water immersion}

Being equipped with a framework to handle more complex cases, two high-NA objectives with built-in correction mechanisms are evaluated in this section. One of them is a NA1.3 glycerol-immersion $(n=1.46)$ objective and the second is a NA1.2 waterimmersion objective. Both of them are corrected for a glass cover slip and feature a correction collar to compensate for sample-induced spherical aberrations. For the simulations, a \#1.5 $(170 \mu \mathrm{m})$ coverslip was added over the settings of the previous chapter. As no total internal reflection will occur for these systems and the correction for spherical aberrations is assumed to be perfect, the performance of the objectives is expected to be uniform throughout the addressable depth range, if the collar is adjusted properly ${ }^{1}$. However, besides absolute performance parameters as resolution and signal levels, it is important for the experimentalist to know the sensitivity of these parameters to nonoptimum phase correction, which is likely to be the case in real-world setups. This was assessed by evaluating the effect of mismatches between the actual imaging depth and the depth that the correction collar is adjusted to.

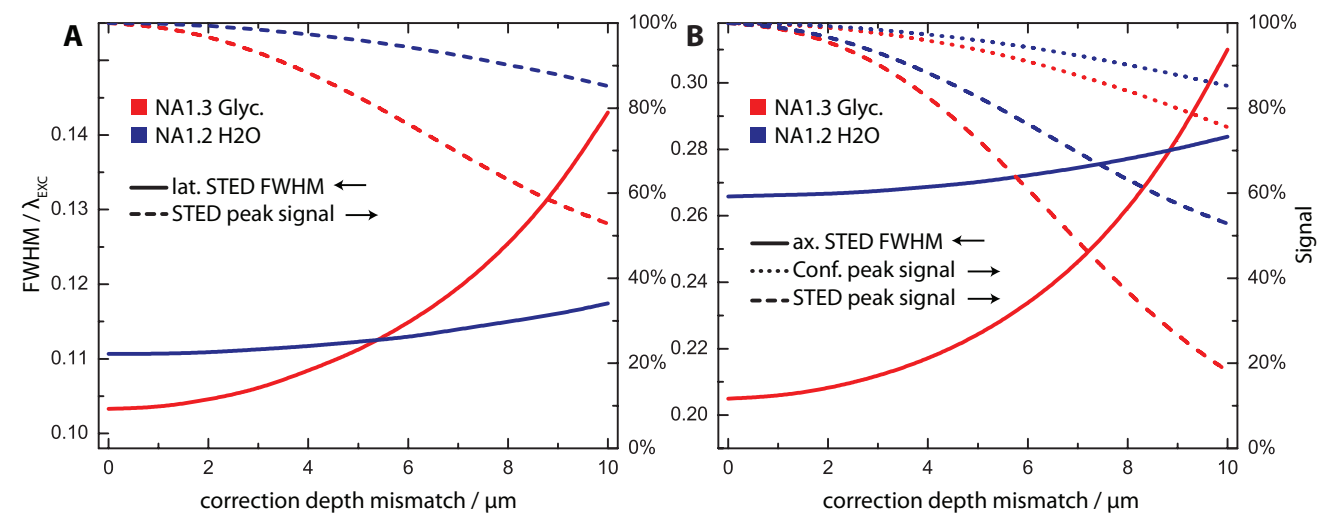

Fig. 2.7: If the correction collar setting and the actual NFP are not perfectly matched, loss of signal and resolution will occur. The sensitivity of the two objectives to non-optimal alignment in STED microscopy is evaluated here for the vortex phase-plate (A) and that used for 3D-STED (B).

The objectives were compared with the same peak intensity of the depletion PSF leading to the same resolution enhancement factor. This is based on the reasoning that the STED resolution in living samples is often not limited by the total available STED laser power alone. As one usually tries to reduce the peak STED intensity in the sample as far as possible to minimize the risk of disturbing the observed organism, the resolution obtainable for a fixed peak intensity is an important key figure for the experimentalist. The initial FWHM offset therefore corresponds to the ratio of the sizes of the diffraction-limited PSFs. In Fig. 2.7, the result is shown for the case of (A) lateral and (B) 3D resolution enhancement. Note that the presented curves are symmetric for positive and negative alignment errors, so only the positive branch is presented here.

\footnotetext{
${ }^{1}$ Fresnel losses are compensated by increasing the power at the back aperture
} 


\section{STED microscopy through planar dielectric interfaces}

In the first case, if the maximum tolerable loss in terms of either resolution or signal is (arbitrarily) defined to be $10 \%$ of the initial value, the glycerol objective has to be aligned within $\pm 4 \mu \mathrm{m}$ of the optimal setting, whereas the water immersion objective tolerates $\pm 8 \mu \mathrm{m}$. The same ratio between the two approximately applies for the $3 \mathrm{D}$ case, with tolerances of $\pm 2 \mu \mathrm{m}$ and $\pm 4 \mu \mathrm{m}$. Note that these ranges also limit the extent of z-stacks that can be recorded without re-adjusting the correction collar.

Generally, the water objective performs significantly better in terms of alignment sensitivity, which is not surprising considering the lower refractive index difference between the immersion medium and the sample. However, this comes at the cost of a lower NA, which reduces the achievable resolution at a given peak intensity, especially along the optical axis. Additionally, one has to take into account how the absolute alignment requirements above translate to the experiment. Usually the correction collar is adjusted by optimizing either the fluorescence signal or the PSF shape while scanning a small structure in the sample in axial direction. It is clear that if changes are harder to sense, the alignment cannot necessarily be performed at a higher absolute precision. Considering mechanical sensitivity, the full scale of the correction collar of the glycerol objective was experimentally determined to correspond to a correction range of $100 \mu \mathrm{m}$ in the present setting. With ten scale parts on the collar, the highest alignment requirement is therefore a little less than half a scale part, which is not difficult to achieve. For the water immersion objective, on the other hand, the full correction range corresponds to a significantly larger range $(>150 \mu \mathrm{m})$, meaning that the required mechanical precision is about of the same order. For all practical matters, the two objectives should therefore be similarly well suited to image through layers of brain tissue.

\subsection{Coverslip tilt}

Apart from the spherical aberrations introduced by the layered structure of the sample, a tilt of the coverslip with respect to the focal plane of the objective can potentially introduce severe aberrations as well. Breaking the rotational symmetry of the system, tilted interfaces are expected to influence the phase pattern of the depletion beam in a totally different way as compared to the cases studied above. To start with, this is not expected to be an issue in standard commercial microscopes due to the perfect initial alignment of the microscope objective and the sample stage. Furthermore, a normal sample consisting of a coverslip cemented to a flat glass slide is intrinsically very planar, which is also true for the bottom of a petri dish. It is therefore not surprising that almost no attention was paid to this problem, while aberrations induced by planar interfaces have been studied very thoroughly in the past. However, when dealing with a macroscopic sample as a whole living animal, this condition cannot be taken for granted. Therefore, the sensitivity of STED microscopy towards coverslip tilt is assessed in this section to find the requirements imposed on the following experiments. 


\subsubsection{Introducing tilt to the numerical model}

As stated above, very little effort has been undertaken in the past to account for a tilted stratified sample medium. In fact, only a single peer-reviewed article was found to address this case numerically [75]. The undisclosed, simple model being outlined therein is stated to rely on the geometrical comparison of optical path lengths and is unlikely to include rigorous vector field simulation. In order to make quantitative predictions with the same precision as for the coplanar case, the theoretical framework developed in this chapter so far is expanded in a simple way to introduce the tilt angle. Instead of

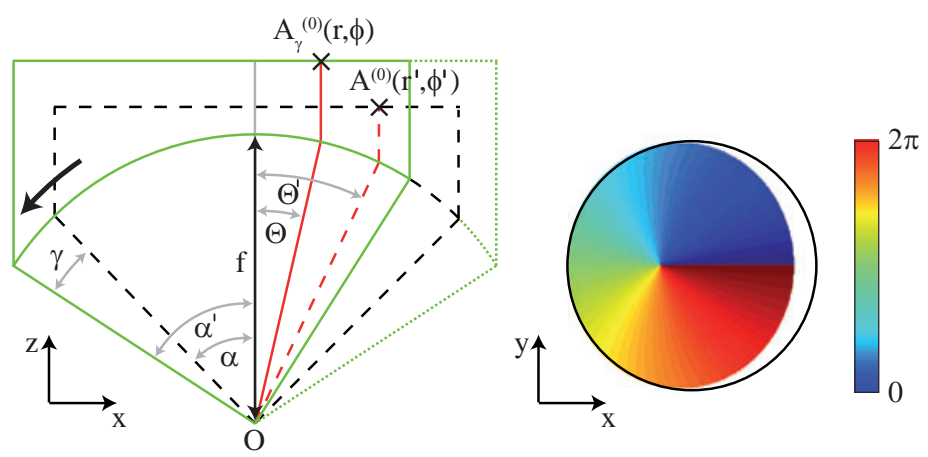

Fig. 2.8: Coverslip tilt by an angle $\gamma$ is implemented by effectively rotating the objective around the origin $\mathrm{O}$ by the same angle. The corresponding pupil function $A_{\gamma}^{(0)}$ obtained is by transformation of the polar coordinates $(r, \phi)$ back to the original coordinate system of $A^{(0)}$. The example on the right depicts the transformed phase of a vortex-patterned pupil function for a large tilt angle of 5 degrees. The white area corresponds to an amplitude of zero, which is omitted in the integration.

modifying (2.9) to account for tilted interfaces, tilt is modeled as a transformation of the pupil function, which is possible because no assumptions were made regarding the symmetry of $A^{(0)}$. As sketched in Fig. 2.8, the proposed transformation corresponds to a rotation of the initial spherical wavefront around the origin $O$ by the tilt angle $\gamma$. The azimuthal integration limit is expanded from the semi-aperture angle $\alpha$ to $\alpha^{\prime}=\alpha+\gamma$ so the new wavefront is not clipped by the former physical aperture cone. The transformed pupil function $A_{\gamma}^{(0)}(r, \phi)$ corresponds to the initial pupil function $A^{(0)}$ evaluated at the transformed coordinates $\left(r^{\prime}, \phi^{\prime}\right)$ :

$$
r^{\prime}=\sqrt{x^{\prime 2}+y^{\prime 2}} \quad \phi^{\prime}=\arctan 2\left(x^{\prime} / y^{\prime}\right)
$$




$$
\begin{aligned}
\left(\begin{array}{l}
x^{\prime} \\
y^{\prime}
\end{array}\right) & =f \cdot \mathbf{P}_{\mathbf{x y}} \mathbf{R}_{\mathbf{y}}(-\gamma)\left(\begin{array}{c}
\sin \Theta \cos \phi \\
\sin \Theta \sin \phi \\
\cos \Theta
\end{array}\right) \\
& =f \cdot\left(\begin{array}{c}
\cos \gamma \sin \Theta \cos \phi-\sin \gamma \cos \Theta \\
\sin \Theta \sin \phi
\end{array}\right) \\
& =r \cdot\left(\begin{array}{c}
\cos \gamma \cos \phi-\sin \gamma \sqrt{f^{2} / r^{2}-1} \\
\sin \phi
\end{array}\right)
\end{aligned}
$$

To find the transformed coordinates, the orthographic projection of $(x, y)=(r \sin \phi, r \cos \phi)$ onto the spherical wavefront with radius $\mathrm{f}$ is first rotated backwards around the $\mathrm{y}$-axis by $\mathbf{R}_{\mathbf{y}}(-\gamma)$ and then projected back to the pupil plane (xy) by $\mathbf{P}_{\mathbf{x y}}$. Thereby the relation $r=f \sin \Theta$ is used to relate polar and spherical coordinates. Note that all operations are performed in cartesian coordinates in order to simplify the rotation about a cartesic axis. All points that are not situated within the original integration limit $\alpha$ after transformation are set to zero amplitude, as they lie outside of the physical aperture. The apodization factor $\sqrt{\cos \Theta}$ in (2.12) reflects a property of the lens, therefore it has to be replaced by $\sqrt{\cos \Theta^{\prime}}$ with $\sin \Theta^{\prime}=r^{\prime} / f$. Notably, all variables that are related to the coordinate system of the objective have to be transformed this way, most prominently the phase correction terms (2.16). As a consequence, the phase correction which is applied in the objective for $\Theta^{\prime}$ is now applied for a plane wave that would actually need a correction for the phase error at $\Theta$. This angle-dependent mismatch between the applied correction and the actual phase error is the main source for aberrations due to tilt.

Sanity checks were performed by numerically evaluating $A_{\gamma}^{(0)}$ for the undisturbed case $\left(n_{1}=n_{2}=n_{3}\right)$ where $\gamma \neq 0$. The obtained focal fields were found to be identical with those resulting in the coplanar case after subsequent rotation by $-\gamma$ about $\mathbf{O}$.

\subsubsection{Effects of coverslip tilt on 2D STED microscopy}

First, the impact of coverslip tilt on the PSF formed by the vortex phase distribution is evaluated. The coverslip thickness is again set to $170 \mu \mathrm{m}$ and the phase corrections for the coverslip as well as for the sample-induced aberrations are applied in the coordinate system of the tilted objective for an NFP of $10 \mu \mathrm{m}$ inside the sample. When looking at the numerical results in Fig. 2.9A, it can be immediately seen that coverslip tilt potentially deteriorates imaging performance on a similar scale as spherical aberration does. It is therefore important to understand the role of the immersion system in the formation of this aberration. When the coverslip is tilted, the angle-dependent geometrical path lengths through the different material layers change and are therefore not matched by the applied correction terms any more. The magnitude of the phase error resulting from this mismatch is proportional to the magnitude of the phase correction term applied at the objective. As a consequence, microscope objectives correcting for larger refractive index differences throughout the layers in the sample space are more susceptible to this aberration than objectives for immersion systems requiring less correction. As the correction term for the coverslip is relatively large due to the thickness and high 

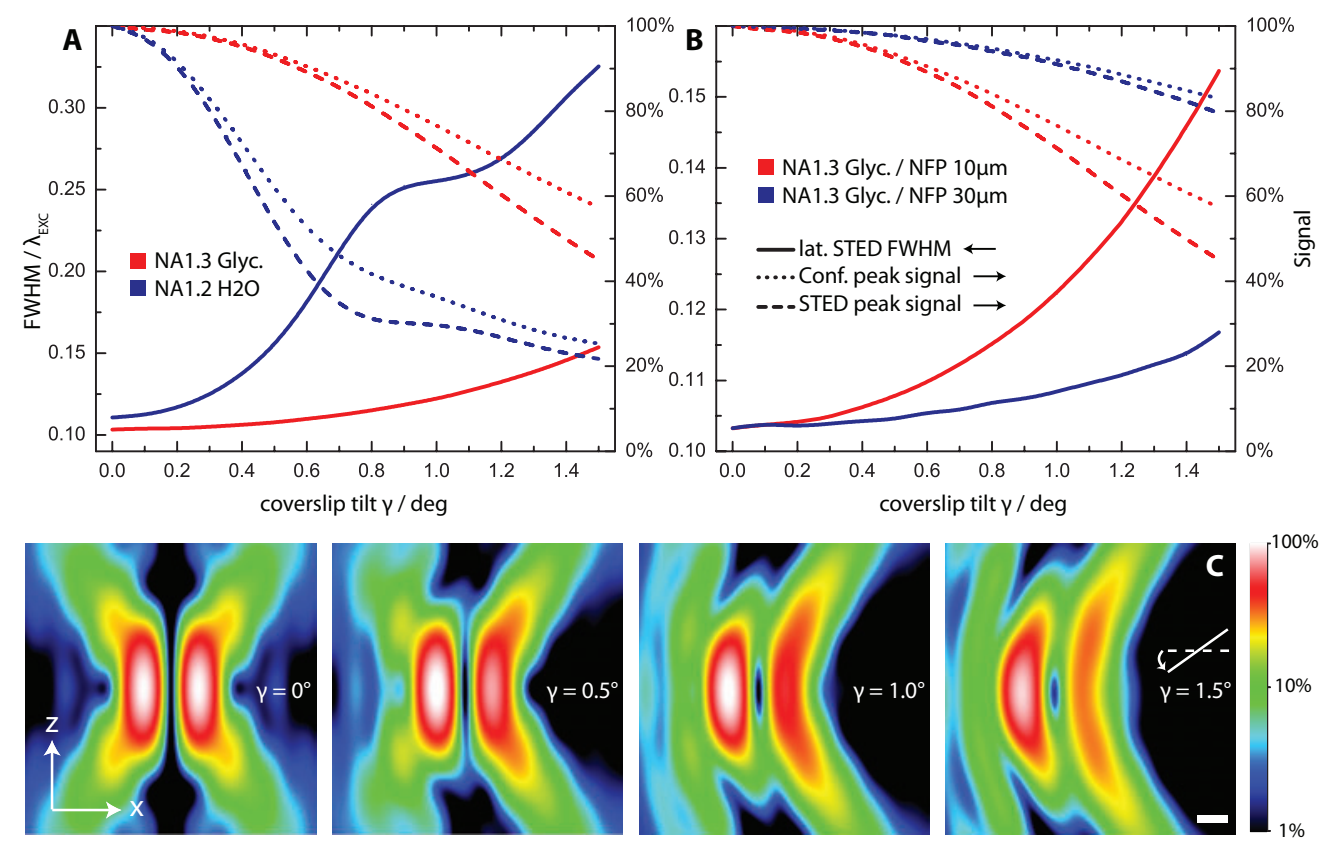

Fig. 2.9: If the glass coverslip $(n=1.515)$ is not oriented in parallel with the focal plane but tilted by an angle $\gamma$, STED resolution and peak fluorescence signal are affected. Water immersion systems are more susceptible to this aberration than glycerol or oil immersion, as shown for an imaging depth (NFP) of $10 \mu \mathrm{m}$ (A). For glycerol objectives, the effect of tilted coverslips decreases with increasing imaging depth in the sample (B). Typical aberrated vortex-phase STED PSFs are depicted in (C) for increasing tilt angles of the coverslip. The PSFs correspond to the series for the glycerol objective in (A). Scale bar is $200 \mathrm{~nm}$.

refractive index of the glass, it plays the most prominent role in the formation of this aberration at NFPs which are small compared to the coverslip thickness. As a consequence, water immersion objectives are more sensitive to tilt-induced aberrations than glycerol objectives. This is because the refractive index difference between the immersion medium and the glass is larger and therefore the magnitude of phase correction is necessarily larger (Fig. 2.9A). On the other hand, in cases where the refractive indices of the immersion medium and the coverslip match, tilt should show almost no effect. In the case of oil-immersion objectives (Sec. 2.2.3) this was experimentally shown to be true [75]. Interestingly, the sensitivity to coverslip tilt even decreases with increasing imaging depth for the glycerol objective (Fig. 2.9B). As the phase correction applied for the coverslip and the sample is of opposite sign, the total phase correction term decreases up to a certain depth, which again reduces sensitivity to tilt. For a water immersion objective the opposite applies, so the sensitivity even increases at greater imaging depth, which should be kept in mind when imaging at extreme depths of up to $1 \mathrm{~mm}$ with coverslip-corrected water immersion objectives [16].

Exemplary PSFs calculated for the NA1.3 glycerol objective at different coverslip tilt angles are shown in Fig. 2.9C. The doughnut-shaped PSF is remarkably stable even for this asymmetric aberration and the intensity at the minimum remains relatively 
small. However, if a tolerance of $10 \%$ signal loss is taken again for STED imaging, the coverslip has to be aligned to within $\pm 0.5 \mathrm{deg}$. for the glycerol objective and, more challenging, to within $\pm 0.2 \mathrm{deg}$. in case of the NA1.2 water immersion objective.

\subsubsection{Aberrations in 3D STED resulting from coverslip tilt}

The last case to be discussed in this chapter aims to assess the impact of a tilted coverslip on the STED PSF for axial resolution improvement. Again, the 3D-STED PSF shows to be impacted stronger by aberrations (note the shorter axis extent for the tilt angle). As can be concluded from the evolution of the fluorescence signal compared to the confocal counterpart with the depletion beam switched off, intensity is quickly accumulating at the minimum of the STED PSF (Fig. 2.10A).
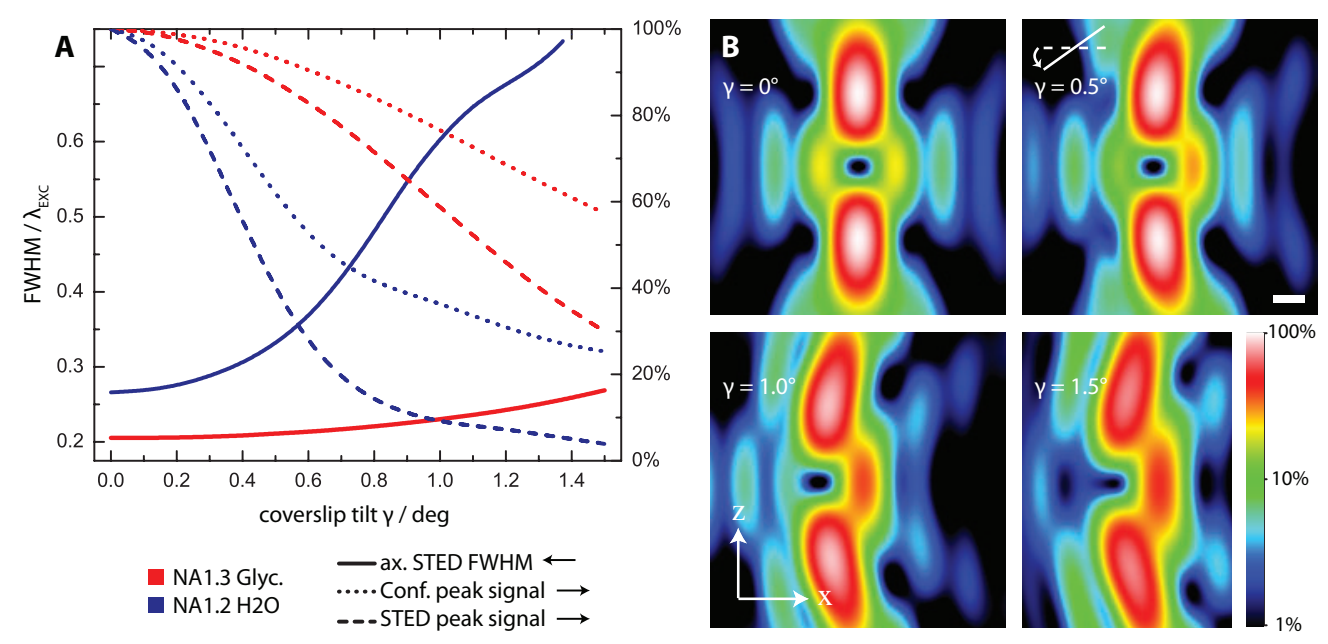

Fig. 2.10: For 3D-enhanced resolution with STED, the effect of coverslip tilt is significantly more pronounced as compared to the doughnut-shaped PSF (A). This can be directly seen from the PSFs calculated for a glycerol immersion objective at increasing tilt angles (B). Scale bar is $200 \mathrm{~nm}$.

The tolerance towards tilt for the case of the glycerol objective here is about $\pm 0.4 \mathrm{deg}$., while less than $\pm 0.2 \mathrm{deg}$ should be achieved for the water immersion objective. Even if these numbers are similar to the $2 \mathrm{D}$ case discussed before, it is clear that $3 \mathrm{D}$ resolution enhancement will be a lot more challenging as possibilities to compensate for signal loss by increasing the excitation power are limited. In order to leave tolerances for other aberrations, the tilt error should therefore be eliminated to a large degree as it can generally by avoided as opposed to the refractive index discussed before (Sec. 2.3.1). 


\section{STED microscopy through planar dielectric interfaces}

\subsection{Summary}

As expected, using high-NA oil-immersion objectives inside living brain tissue should be avoided due to the severe aberrations present in this setting. Only the very surface of a sample can be imaged in this way with acceptable performance. This is not different for STED microscopy. In this chapter, it was shown that modern glycerol and water immersion objectives are much better suited for this task, as they can be adjusted to correct for the spherical aberrations caused by the refractive index mismatch between the sample and the immersion medium. For STED microscopy, this is even more important as the relative loss in resolution is in some cases more pronounced than in an ordinary CLSM under equal experimental conditions. However, when aberrations are carefully compensated, STED shows to be remarkably stable, despite the complex phase patterns employed for the depletion beam. Although the STED PSF for axial resolution improvement tends to require more attention than the one based on a vortex phase distribution, 3D imaging should also be feasible with either glycerol or water immersion.

An additional challenge introduced by these immersion systems though, is the high sensitivity to coverslip tilt, which has been numerically quantified here for the first time. In order to obtain optimal STED images, it was shown that the planarity of the coverslip with respect to the focal plane has to be assured in the experiment to within a few mrad. In order to avoid problems associated with coverslip tilt, it might sound tempting to avoid the problems associated with coverslips altogether by employing so-called dipping objectives which are directly immersed into a pool of liquid on the sample. However, one has to keep in mind that the coverslip fulfills two important functions in the experiment. Apart from sealing the sample against environmental influences, it also ensures that the interface between the immersion medium and the sample is actually flat, thereby limiting the occurring aberrations to the simple forms discussed in this chapter. If this was not the case, the structured surface of a sample as the brain would additionally cause complex high-order aberrations which vary across the sample. ${ }^{1}$ This point will be addressed again in the next chapter to optimize the sample preparation.

To conclude, either the NA1.2 water immersion objective or the NA1.3 glycerol objective discussed in this chapter make a good choice for imaging inside brain tissue. While the water immersion objective is more sensitive to coverslip tilt than the glycerol objective, the refractive index of water is closer to that of the brain and therefore less spherical aberration is introduced. However, the reason that the glycerol objective was chosen for all of the following work is simply its higher numerical aperture, which enables higher resolution at the same peak intensity of the STED PSF.

\footnotetext{
${ }^{1}$ Ideally, a cover slip having the same refractive index as the immersion medium could be used in conjuction with a dipping objective. The only suitable material currently known having the refractive index of water is a polymer which is distributed under the trademark CYTOP, being exclusively manufactured by Asahi Glass Corp. In fact, a patent for coverslips made from this material is held by Olympus [76], but unfortunately they were never in production and are therefore unavailable.
} 


\section{Experimental methods and techniques}

Following the introduction of the theoretical concepts behind STED microscopy, the experimental methods which enable super-resolution microscopy in vivo are presented in this chapter. In addition to the optical and mechanical design of the STED microscope, the surgical preparation of the mouse and the exact experimental procedure are described in detail. This chapter will thus hopefully serve as a comprehensive guide to those intending to follow up on the experimental results presented in this work.

\subsection{A STED microscope for physiology applications}

An optimized STED microscope was built to address the special requirements imposed by in vivo experiments. First, for physiological reasons, the microscope has to be built in an upright configuration to enable imaging of the cortex of a living subject. This is in contrast to the stringent stability requirements for nanometer-scale imaging, as upright microscopes tend to be more sensitive to vibrations compared to inverse configurations built closer to the top of the optical table. Special attention was therefore paid to rigid construction using cross-bracings wherever possible and filling hollow spaces with heavy granular material for enhanced damping. Secondly, a large sample space is necessary to accomodate whole animals with additional room for preparation and fixation, while providing positioning accuracy of at least a few microns. A schematic drawing of the constructed STED microscope is shown in Fig. 3.1. It is based on a commercial microscope stand (BX51WI, Olympus) that was modified to accommodate a setup for laser-scanning microscopy, including the additional beam path for STED. A motorized flip-mirror is used to switch between wide-field observation and laser-scanning mode. Fluorescence wide-field images were additionally recorded with a cooled CCD camera (3MP TCC-3.3ICE-N, Tucsen). The OEM sample stage was replaced with a large stepper-motor driven XY-translation stage (SCAN IM \& TANGO controller, Märzhäuser Wetzlar) mounted on a motorized heavy-duty linear stage (LNR50S/M, Thorlabs) for axial coarse positioning. In this way a total sample volume of 122 x 88 $\mathrm{x} 50 \mathrm{~mm}(\mathrm{XYZ})$ could be accessed with sub-micron precision in all spatial directions and excellent resistance towards vibration and drift.

The STED light with a wavelength of $592 \mathrm{~nm}$ is provided by a frequency-doubled optical parametric oscillator (Advanced OPO, APE Berlin), which is synchronously pumped by a mode-locked Titanium-Sapphire laser (Chameleon ULTRA II, Coherent Corp.) running at $792 \mathrm{~nm}$ with a repetition rate of $80 \mathrm{MHz}$. Stabilization and control of the output power is achieved with a liquid-crystal based power controller (LPC-VIS, Brokton Electro-Optics corp.). The femtosecond pulses from the OPO are stretched to 300ps to avoid two-photon excitation in the sample and to improve the interaction 


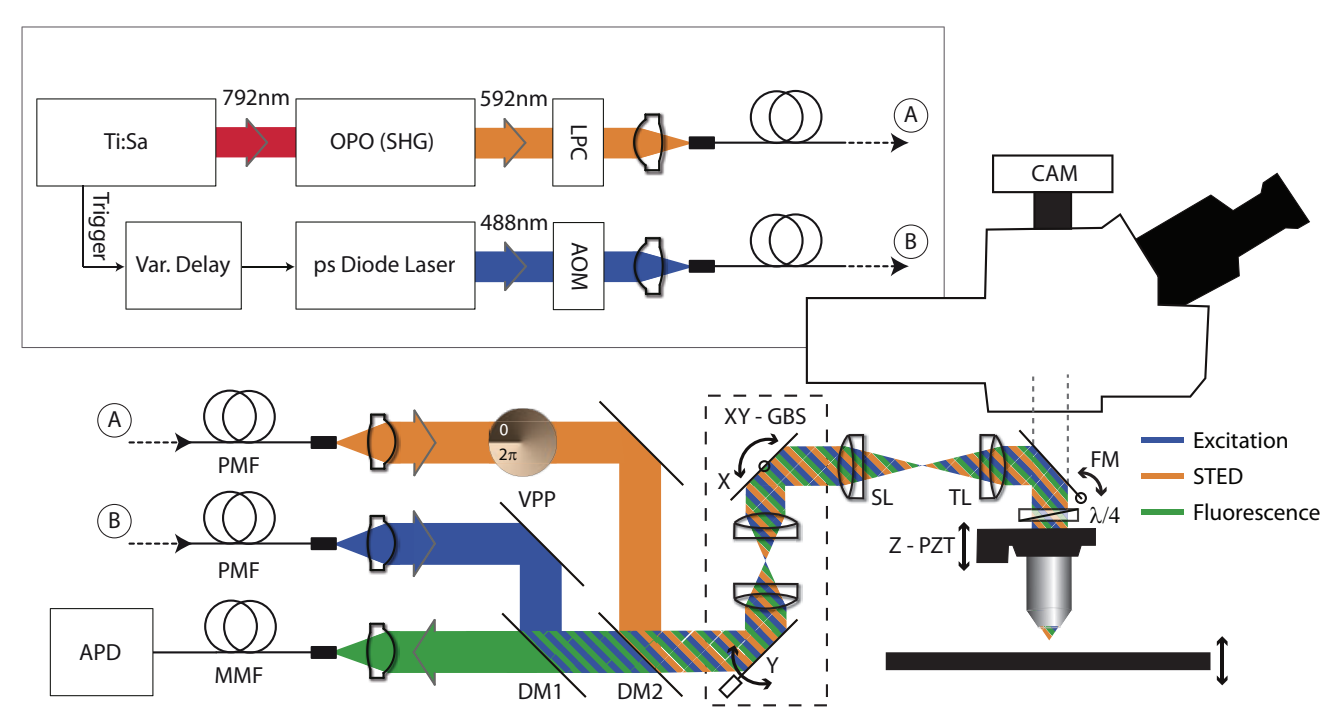

Fig. 3.1: Schematic drawing of the upright STED microscope used for the experiments. $\lambda / 4$ : quarter-waveplate, AOM: acousto-optic modulator, APD: avalanche photo diode, CAM: CCD camera, DM: dichroic mirror, FM: flip-mirror, GBS: galvanometer beam scanner, LPC: laser power controller, MMF: multi-mode optical fiber, OPO: optical parametric oscillator, PMF: polarization-maintaining single-mode optical fiber, PZT: piezo-transducer, SL: scan-lens, Ti:Sa: Titanium-Sapphire fs laser, TL: tube lens, VPP: vortex phase-plate

of the STED light with the dye molecules. This is realized by chirping the pulses in a $120 \mathrm{~m}$ stretch of polarization-maintaining single-mode fiber (488nm PMF, OZ Optics), thereby taking advantage of the broad spectrum of the fs-pulses and the strong dispersion of the silica core in the visible. In order to prevent damage to the fiber, the pulses are first stretched to a few ps, by passing them through two $30 \mathrm{~cm}$ long glass rods made of highly dispersive SF6 glass. The excitation light at $488 \mathrm{~nm}$ is generated by a pulsed diode laser (picoTA 488, PicoQuant Berlin) emitting pulses with a length of 100ps. The laser beam passes through an acousto-optic modulator (AA-AOTF-nC-TN, AA Optoelectronics Orsay) to control the beam power and is then coupled to a short polarization-maintaining fiber for spatial mode cleaning and facilitated handling. The pulses of the STED laser have to arrive at the sample within a few picoseconds after the excitation pulses to ensure optimal suppression of the fluorescence. This is achieved by delaying the reference signal of the Ti:Sa oscillator through a variable electronic delay stage (custom-built) before applying it to the trigger input of the diode laser controller.

At the microscope, the STED light emerging from the fiber is collimated and guided through a phase plate(VPP-1, RPC Photonics Rochester) that applies a helical phase profile to the wavefront. This is technically achieved with a polymer pattern of varying thickness, which retards parts of the wavefront with respect to others. Continuing from there, the STED beam is combined co-axially with the excitation beam on a short-pass dichroic mirror (Z590SPRDC/5mm substrate, Chroma Technology, Bellows Falls/VT). Both beams are then deflected by a commercial beam scanning unit (YANUS IV, Till Photonics) featuring two fast galvanometer mirrors and mirror-based relay optics imag- 


\section{Experimental methods and techniques}

ing one scan mirror onto the other in a 4f-configuration. This is important to make sure that the beam is not laterally shifted in the pupil plane when scanning. Afterwards, the deflected beams pass through a matched combination of scan- and tubelens (Leica Microsystems, Wetzlar), which are again arranged in a 4f-configuration such that the second deflection mirror is in the conjugate plane of the back aperture of the objective. The microscope objective (63x/1.30 GLYC CORR CS, Leica Microsystems, Wetzlar) is mounted on a piezo transducer with $100 \mu \mathrm{m}$ travel range(MiPOS $100 \mathrm{PL}$ CAP, piezosystem Jena, Jena), to enable axial fine-positioning and scanning within the sample. A second objective with a lower NA (UPlanFL N 10x/0.30, Olympus, Tokyo) is co-mounted with the afore mentioned one on a special swing nose-piece (WI-SRE3, Olympus, Tokyo) to enable switching between high-NA CLSM/STED scanning of a small region and a wider field-of-view for coarse orientation.

Fluorescence from the reduced focal area is collected by the same objective and descanned when passing through the beam scanning unit in reverse. It is separated from the excitation light with a long-pass dichroic mirror (Z488RDC, Chroma Technology, Bellows Falls/VT) and focussed into a multi-mode optical fiber with a core diameter of $62.5 \mu \mathrm{m}$ after passing a bandpass filter (HQ530/60, Chroma Technology, Bellows Falls/VT). The core of the fiber acts as a confocal pinhole in this setting, with a diameter corresponding to one Airy-disc diameter of the excitation wavelength. A fiber-coupled avalanche photo diode (SPCM-AQRH-13-FC, Excelitas Technologies Corp.) operating in Geiger-mode detects the fluorescence at the end of the multimodefiber, creating an electronic pulse for every detected photon. The galvanometer mirrors as well as the objective piezo translator are controlled with the analog outputs of a general-purpose PCIexpress data acquisition card (X-Series DAQ PCIe-6353, National Instruments, Austin/TX), which is also used to count the pulses from the APD. On the host PC, the acquisition process and rendering of the recorded images is controlled by the microscopy software Imspector (www.imspector.de), which was custom developed in-house.

\subsection{Dual-color STED imaging with EGFP and EYFP}

Whenever multiple laser wavelengths are used in a laser scanning microscope to separate populations of different fluorophores, care has to be taken to chose the right laser lines for the right set of dyes, otherwise cross-talk between the channels can deteriorate the results. This obviously gets harder when the number of laser lines increases, which is why the depletion lasers in a STED microscope pose an additional challenge to multicolor imaging. One has to bear in mind that even a small excitatory cross-section of any of the dyes at the STED wavelength will result in severe cross-talk due to the $10^{5}$ times higher light intensity compared to the excitation lasers. During the last couple of years, however, a couple of techniques have been employed to allow simultaneous recording of two fluorescent markers. The most obvious approach is the introduction of a separate pair of STED and excitation lasers for the second dye, which has been shown to work if the spectra of the dyes show only little overlap [77, 78]. Even in this case though, the channels are recorded sequentially starting with the red channel, which would otherwise be bleached immediately by the STED laser with the smaller 


\section{Experimental methods and techniques}

wavelength. Apart from making the optical alignment more complex, the second STED laser also limits co-localization accuracy, as the alignment of the two channels depends crucially on the precise overlap of the zero-intensity spots of the depletion PSFs and is thus usually limited to $5-10 \mathrm{~nm}$. Also, no combination of fluorescent proteins (FPs) was found to work in this constellation yet. Separating the labels by fluorescence lifetime as in [79] is also not option for FPs, as the required lifetime difference of $>1 \mathrm{~ns}$ is usually not given. The only method proven so far to work with FPs relies on the use of two reversibly photo-switchable fluorescent proteins (RSFPs) [80]. As the two proteins show opposite switching kinetics upon irradiation with UV light, they can be sequentially recorded in a monochromatic STED setup. The disadvantage is currently a lack of transgenic animals expressing these proteins. Also, using a UV laser could reduce the penetration depth into the sample, due to increased scattering cross-sections at smaller wavelengths,

The method implemented here can be seen as a generalization of the one presented in [81], where two dyes with distinct excitation spectra are required of which the bluer one possesses an extraordinary large Stokes shift. In this way, the same STED laser can be used for both dyes while sequential excitation with different lasers allows for separation of the channels. Besides the simplicity of this approach, having a common STED beam also guarantees highest co-localization accuracy between the channels, as the location of the fluorescent spot is only defined by the zero of the depletion PSF at high saturation factors. Although this methods is not directly applicable to fluorescent proteins, where currently no variant with a sufficiently large Stokes shift is known, two closely related FPs with similar emission spectra could still be depleted with the same STED laser. As it turns out, the FPs EGFP and EYFP can both be efficiently switched off with a wavelength of $590 \mathrm{~nm}$, which makes them a good choice for this scheme. Additionally, they are already expressed in many existing transgenic mouse models, which would otherwise take years to breed. The flip-side of this approach is certainly the proximity of the respective emission maxima, which are situated only $20 \mathrm{~nm}$ apart $(509 / 529 \mathrm{~nm})$. In order to separate these largely overlapping spectra, a carefully designed combination of excitation and detection bands was implemented according to Fig. 3.2. Only differences to the previous design shown in Fig. 3.1 are presented here. One of the key components is the picosecond-pulsed diode laser emitting at $518 \mathrm{~nm}$ (LDH-510, wavelength-selected, PicoQuant Berlin) which has just recently become available and allows for selective excitation of EYFP close to its maximum crosssection with a contrast of 10:1 over EGFP. This laser is reflected off the dichroic mirror DM3 (RazorEdge LP02-514RU-25, Semrock), which has a very steep separation edge and which was angle-tuned for perfect reflection (>OD6) of the $518 \mathrm{~nm}$ laser line. EGFP is excited with a similar contrast by the second pulsed diode laser at $440 \mathrm{~nm}$ (LDH-P-C-440M, PicoQuant Berlin), which is overlaid at the blue end of the spectrum via the long-pass dichroic DM2 (Z450RDC, Chroma Technology, Bellows Falls/VT). Note that only longpass dichroic mirrors are used in this design as they usually have steeper edges compared to their shortpass equivalents. In order to further enhance discrimination of the two FPs, the detection band is also split at DM3 and directed to different detectors. The blue part of the spectrum is separated from the $518 \mathrm{~nm}$ excitation beam at DM4 (Z525DCXR, Chroma Technology, Bellows Falls/VT) while the greener part is simply transmitted through DM3. After bandpass filtering (HQ490/40 


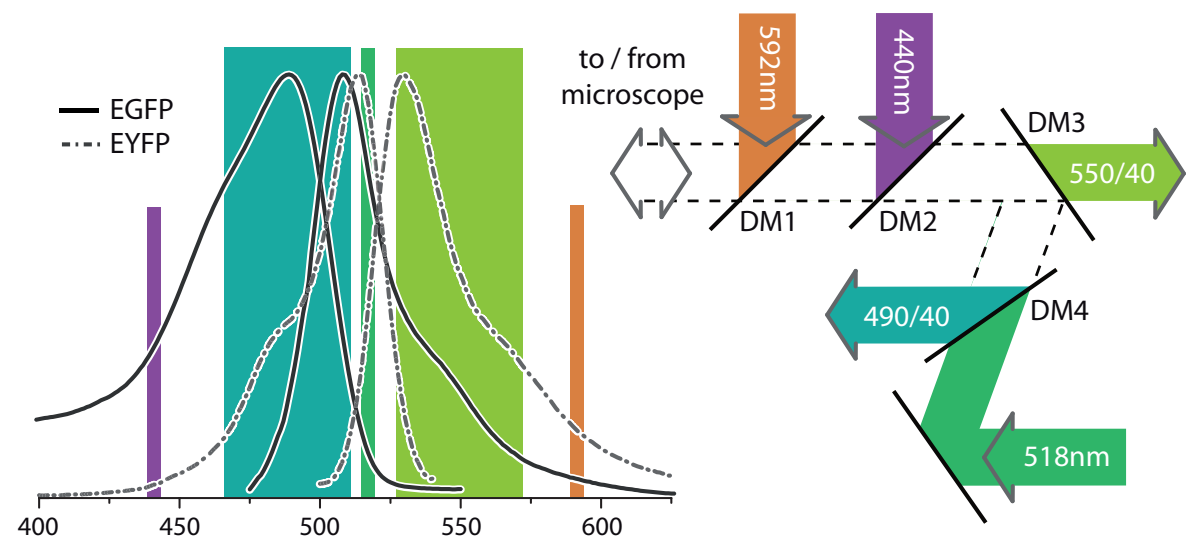

Fig. 3.2: The illumination scheme of the STED microscope is altered to allow dual-color superresolved imaging of EGFP and EYFP. Four dichroic mirrors (DM) are used to separate the spectrum into three laser beam paths and two detection bands. Elements in common with the previously presented setup have been omitted.

and HQ550/40, Chroma Technology, Bellows Falls/VT), about $60 \%$ of the detected fluorescence photons emitted by EGFP end up in the blue detection channel and $40 \%$ in the green detection channel. More than $95 \%$ of the detected light originating from EYFP is observed in the green channel compared to less $5 \%$ in the blue channel.

In order to separate the two labels, the excitation beams have to be toggled during recording, which is usually performed on subsequent scans of the same line or the frame. In living systems however, this can lead to wrong interpretation of the data as movements taking place on a faster timescale than the switching period will lead to relative local displacements of the channels. While alternation of the excitation lasers at the pixel level is possible, it gets increasingly difficult to implement as pixel dwell times get shorter and approach the transient response times of AOMs, which are among the fastest devices available for switching. An elegant solution is to use pulseinterleaved excitation [82], where the excitation wavelength changes at every pulse cycle and the alternation timescale is therefore in the nanosecond regime. This is especially important for STED microscopy, where much less local misalignment can be tolerated due to the higher imaging resolution compared to regular CLSM.

In Fig. 3.3, the complete timing scheme and the according electronic triggering chain is illustrated. Care has to be taken to synchronize the excitation pulses of both wavelengths with the pulses of the STED laser. The $80 \mathrm{MHz}$ sync signal from the Ti:Sa laser is first divided by 2 with an ECL logic chip (M10ECL32, ON Semiconductor) and fed into a dual-channel variable electronic delay stage (custom-designed) allowing for independent delays to be applied to its outputs. These delays are adjusted such that the pulses of the two diode lasers arrive at the sample sequentially and with optimal timing with respect to the STED pulses for best depletion efficiency. The sync signals of these lasers are then provided as start signals to fast electronic gates ${ }^{1}$ which forward only those pulses from the APD detectors to a specific output arriving within a certain timespan after the start signal. In this way, four virtual output channels are generated

\footnotetext{
${ }^{1}$ The gating electronics were custom designed in-house, but commercially available time-correlated single photon counting (TCSPC) cards could be used instead
} 

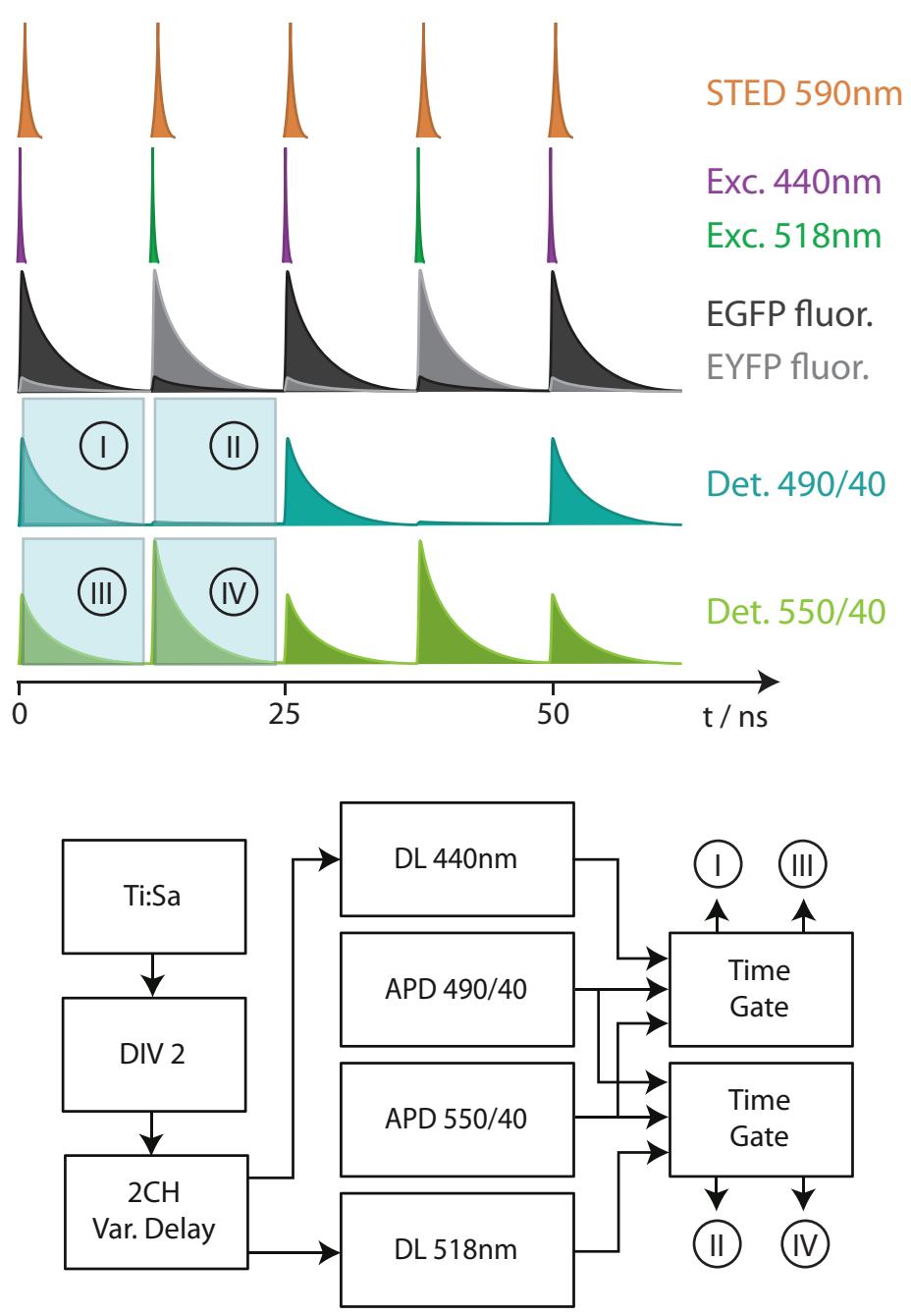

Fig. 3.3: Timing scheme used for STED microscopy with pulsed-interleaved excitation and two detection channels. The area under the fluorescence decay curves reflects the ratios between the photon numbers in the different channels. The block diagram in the lower part shows the trigger chain for electronic synchronization and the timegating devices which are used to derive the four virtual detection channels I-IV.

according to the timing intervals in the diagram in Fig. 3.3. Note that at the present pulse period of $12.5 \mathrm{~ns}$ the residual fluorescence at the beginning of the subsequent cycle is only about $1 \%^{1}$, so no cross-talk is expected between the gating channels acting on a single detector. As a result, channel I and channel IV separate EGFP and EYFP with virtually no cross-talk, which allows for linear unmixing of the contributions found in channel III. All outputs are simultaneously recorded with counters on the DAQ board, including channel II for control. Note that this channel should detect practically no photons in this configuration. It could therefore potentially be used to discriminate an additional blue-shifted FP such as CFP in the future by adding a UV excitation laser.

${ }^{1}$ The fluorescence lifetime of both fluorescent proteins was determined to be about $2.8 \mathrm{~ns}$ 


\subsection{Experimental procedure}

Special care was taken during preparation of the mouse to prevent motion resulting from respiratory or cardiovascular activity. This is especially important for superresolution microscopy where even the slightest movements on the order of a few $\mathrm{nm}$ will cause imaging artifacts. The optimized preparation techniques shown here can be used to passively suppress intrinsic motion almost completely. Technically more sophisticated methods such as numerical de-warping [83], synchronization of image acquisition and heart beat [84], active stabilization via a servo-actuated feed-back loop [85] or even cardio-pulmonary bypassing [86] are therefore not necessary.

The experiments were performed according to the ethics guidelines of national law regarding animal protection procedures and were authorized by the ethics committee of the Max-Planck-Institute for Biophysical Chemistry and by the responsible authorities, the Niedersächsisches Landesamt für Verbraucherschutz.

\subsubsection{Anesthesia and surgery}

In mice aged 7 weeks to 18 months (weight range, 19-35 g), general anesthesia was initiated by $60-80 \mathrm{mg}$ pentobarbital sodium i. p. (in $0.9 \% \mathrm{NaCl}$ ) per $\mathrm{kg}$ body weight. In supine position, the jugular vein was cannulated, and anesthesia was continued i.v. with $40-60 \mathrm{mg} /(\mathrm{kg} * \mathrm{~h})$ methohexital sodium (Brevimytal, Hikma, London), dissolved in $0.9 \% \mathrm{NaCl}$, throughout the whole experiment. A tracheotomy was performed, and a tracheal tube was inserted for artificial ventilation. After closing the neck wound with skin closure clips, the mouse was positioned in prone position. To avoid movements induced by active respiration during imaging, mice were paralyzed with pancuronium bromide (Pancuronium-Actavis, Actavis Deutschland $\mathrm{GmbH}$, Langenfeld), and artificially ventilated with a gas mixture of $\mathrm{CO}_{2}(2.5 \%), \mathrm{O}_{2}(47.5 \%)$, and $\mathrm{N}_{2}(50 \%)$ at $60-120$ strokes/min (100-140 $\mu 1 /$ stroke depending on the oxygenation of the blood).

The detailed steps for the preparation of the cranial window can be seen in Fig. 3.4. After removing the skin from the skull, a flat holder which could be tilted in either direction was fixed with dental cement (Durelon ${ }^{\circledR}, 3 \mathrm{M}$ ESPE, Seefeld, Germany) on the skull about $5 \mathrm{~mm}$ frontal to the bregma (all anatomical descriptions refer to Paxinos and Franklin [87]). The left skull was trepanned with a micro drill (FST, Heidelberg) between the bregma and the lambdoid suture for a window of about $2 \mathrm{~mm}$ diameter, taking off the boneside layer of the meninges at the same time. The remaining thin cerebral layer of the meninges (Pia Mater) was opened with fine forceps, taking care not to damage the cortical surface and to avoid extended blood cell deposits on the cerebral surface. If left in place, the inhomogeneous collagen structure of this layer causes aberrations that severely affect imaging resolution and brightness.

A coverslip (5 mm diam.) is fixed on the hole to form a closed chamber with a flat part of the cerebral cortex touching the coverslip from underneath. Besides providing a flat interface with the brain, the coverslip also has the important role of mechanically stabilizing the tissue through adhesion. Similar to the method presented in [88], albeit much simpler, a drainage method is introduced here to prevent the formation 


\section{Experimental methods and techniques}
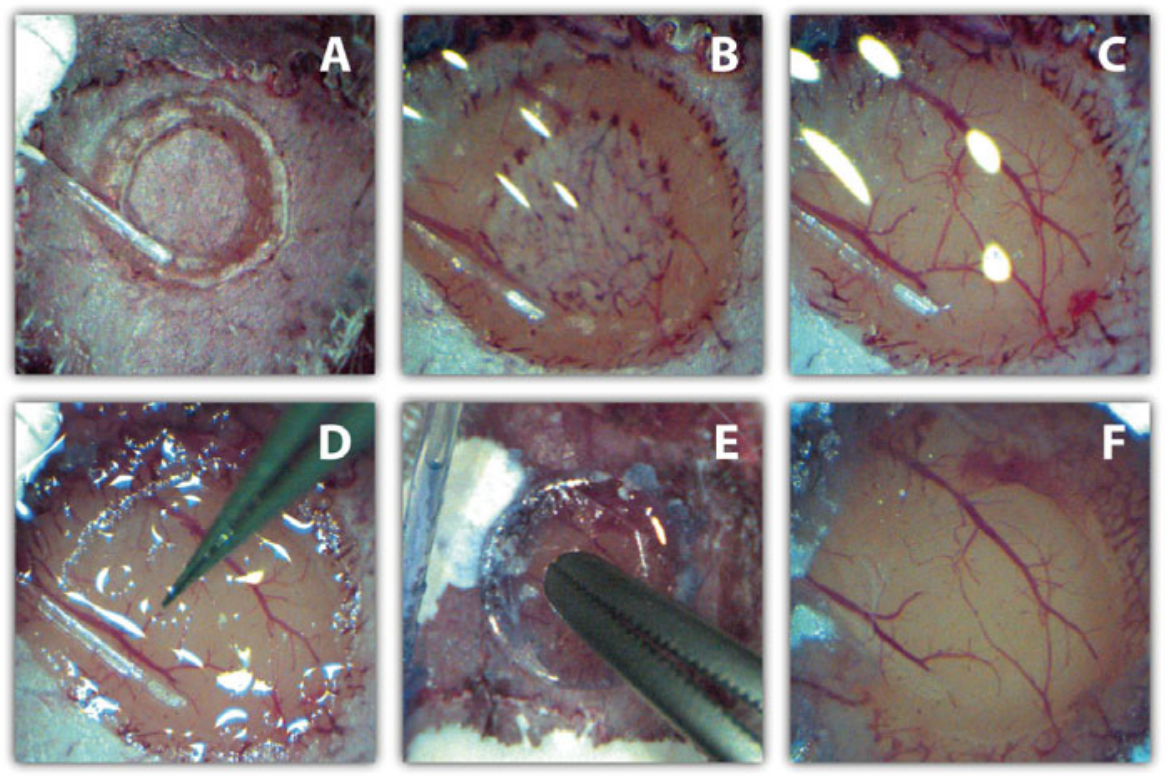

Fig. 3.4: Preparation of the cranial window. After removal of the skin and fixation of the skull, a circular groove is applied and the drainage tubing is fixed with cement (A). The groove is then deepened until the bone becomes transparent (B) and the center part can be lifted off (C). With fine forceps, the Pia is opened (D) before a round glass coverslip is glued directly to the skull with Histoacryl (E). When the protective layer on the glass is removed, the drainage tubing and the outline of the opening in the Pia can be observed $(\mathrm{F})$.

of a cerebrospinal fluid layer between the coverslip and the cortical surface during the experiment. In this way, the brain tissue is always in immediate contact with the glass surface. First, a very fine tubing with an outer diameter of approx. $150 \mu \mathrm{m}$ was pulled from a molten disposable plastic syringe, inspired by a technique used for backfilling micropipettes $[89,90]$. The tubing was then cut at a $45^{\circ}$ angle with a scalpel and introduced via a tangential groove to the aforementioned chamber. Before fixing the coverslip to the skull with Histoacryl (Aesculap, Tuttlingen, Germany), the lower surface was coated with a sparse layer of 40nm fluorescent beads (yellow-green FluoSpheres ${ }^{\circledR}$, Invitrogen, Carlsbad/CA) to render it visible in fluorescence contrast. To prevent glue and body fluids from blurring the upper surface during preparation, a protective polymer (FirstContact ${ }^{\circledR}$, Photonic Cleaning Technologies) was applied in connection with clear scotch film and peeled off just before imaging. Finally, in order to prevent body motion resulting from forced inhalation, a spinal cord clamp (STS-A, Narishige, Tokyo) was introduced through small incisions lateral to the lumbal spinal column (L4) to lift the torso from the support. A sketch of the complete preparation for the imaging procedure is shown in Fig. 3.5. Most of the surgical procedure was performed under a preparation binocular (Leica M60). 


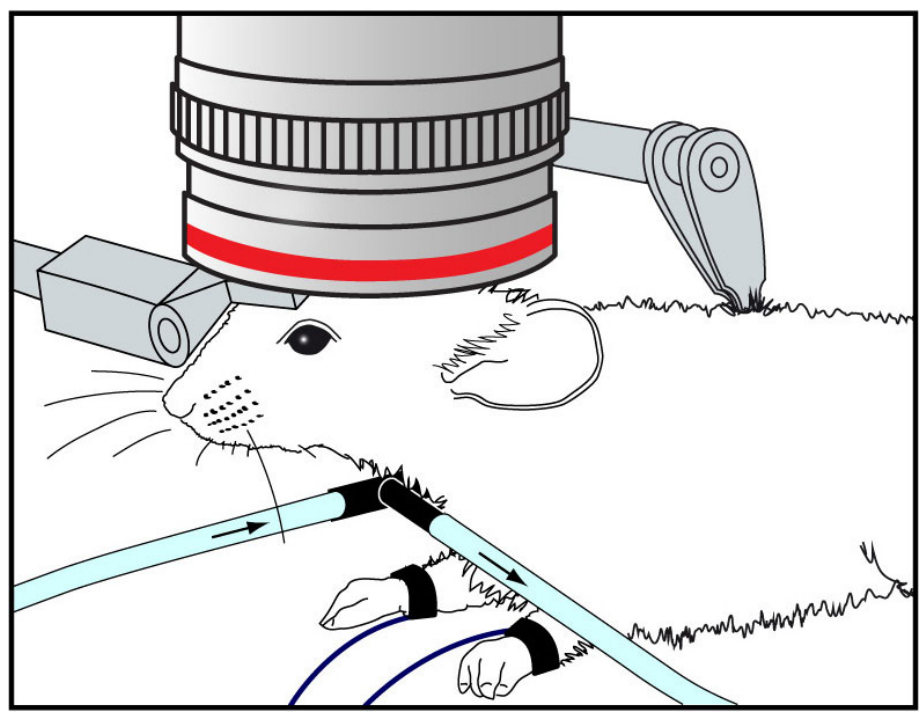

Fig. 3.5: An anesthetized mouse prepared for intravital STED microscopy of the brain. Artificial ventilation is performed via a tracheal tube which is attached to the air in- and outlets at the t-joint visible at the neck. Head and spinal column are attached to adjustable holders mounted on a rail on the sample stage. Vital signs are constantly monitored via ECG electrodes attached to the front paws, a rectal temperature probe and a pulse oxymeter clip positioned at the right thigh (not shown).

\subsubsection{Maintenance of the mouse}

The body temperature was rectally measured (GTH 1170 thermometer/GTF 300 sensor, Greisinger, Regenstauf) and kept between $36^{\circ} \mathrm{C}$ and $38^{\circ} \mathrm{C}$ by a heatable sample support. Electrocardiograms were monitored throughout the experiment. For sufficient anesthesia, the heart rate was kept below $420 \mathrm{~min}^{-1}$ where surgical stress could not be detected any more. Additionally, the oxygenation of the blood was monitored by a pulse oximeter (MouseOx ${ }^{\circledR}$, Starr Life Sciences, Oakmont, PA, USA) and the values were taken to adjust artificial respiration for oxygen saturation above $90 \%$. Following this procedure, the mouse was routinely kept alive under the microscope for more than 8 hours after initiating the anesthesia.

\subsubsection{Image acquisition and analysis}

Shape and alignment of excitation and depletion PSFs were checked at the beginning of every recording session to ensure optimal performance. After preparation, images of the cortical surface vascularization were taken under the preparation binocular for coarse reference. The mouse was then transferred to the microscope sample stage and the region of interest was identified in widefield mode at 10x magnification before switching to the $63 \mathrm{x} / 1.30$ objective. The tilt of the coverslip with respect to the focal plane was corrected with fine-pitch screws attached to the sample plate while observing the fluorescent beads under widefield illumination. As defocus of the beads was visible to within $\pm 100 \mathrm{~nm}$ over a field of view of $100 \mu \mathrm{m}$, the coverslip tilt was effectively 


\section{Experimental methods and techniques}

reduced to $\pm 2 \mathrm{mrad}$ ( $\pm 0.1 \mathrm{deg}$.). Afterwards, the setup was switched to laser-scanning mode and target structures were identified performing fast overview scans. Vicinity to blood vessels and capillaries was avoided as hemoglobin slightly absorbs the orange STED light and occasionally caused thermal damage. The correction collar of the microscope objective was fine-tuned at the desired imaging depth by observing the image of a fine structure such as a thin axon while performing fast scans in the $\mathrm{xz}$ (or yz) plane. Imaging a sub-diffraction feature in this way effectively depicts the confocal PSF, which can then be optimized regarding its axial extent, focal plane symmetry and brightness. This procedure was repeated every time the imaging depth or the imaged region was changed.

For STED imaging, rectangular regions varying from $10 \times 10 \mu \mathrm{m}$ to $30 \times 30 \mu \mathrm{m}$ were raster-scanned with pixel sizes ranging from $20 \mathrm{~nm}$ to $30 \mathrm{~nm}$ at an 1:1 pixel aspect ratio. As most structures of interest are not planar, up to $10 \mu \mathrm{m}$ thick volumes were imaged as stacks of xy-planes spaced 500-600nm apart. The time-average focal power of the excitation lasers was on the order of a $1-10 \mu \mathrm{W}$, while a maximum of $35 \mathrm{~mW}$ was used for the STED laser. Where not indicated differently, images shown in this work were slightly smoothed with a Gaussian profile of 1.0 pixels width to suppress highfrequency noise. All quantitative analysis was performed on the raw data using ImageJ, including the depicted intensity profiles. For visualization of volume data, image stacks were projected to a single plane by maximum intensity projection. Image processing exceeding these steps is mentioned in the context of the respective measurements. 


\section{Experimental results}

\subsection{Dendritic spine plasticity in the adult brain}

Dendritic spines are small protrusions that can be found on the dendrites of most principal neurons in the brain, such as on the pyramidal neurons of the neocortex. The dendrites of a single neuron can easily feature several hundred thousand of these protrusions. They form the post-synaptic part of the majority of excitatory synapses, with the pre-synaptic site mostly located on axons of other neurons. As Ramón y Cajal already depicted in his drawings (Fig. 1.1, Chap. 1), most spines have a pronounced head structure are connected to the dendrite by a thin neck. It is well known that dendritic spines show a high degree of structural plasticity. They can change their shape on timescales of days to minutes and seconds including complete retraction of existing spines and de-novo growth of new ones [91]. Experiments have shown that these structural changes can be linked to synaptic activity ${ }^{1}$ by correlating them to external stimuli [15, 93-97]. Consequently, spines are generally attributed a key role in learning processes and the formation of long-term memory. But even though experimental support for this hypothesis has recently started to emerge [98-100], the mechanisms

${ }^{1}$ Cajal was the first one to surmise that spine morphology and activity could be interdependent:"The state of activity would correspond then, to the swelling and elongation of the spines, and the resting state (sleep or inactivity) to their retraction." [92]
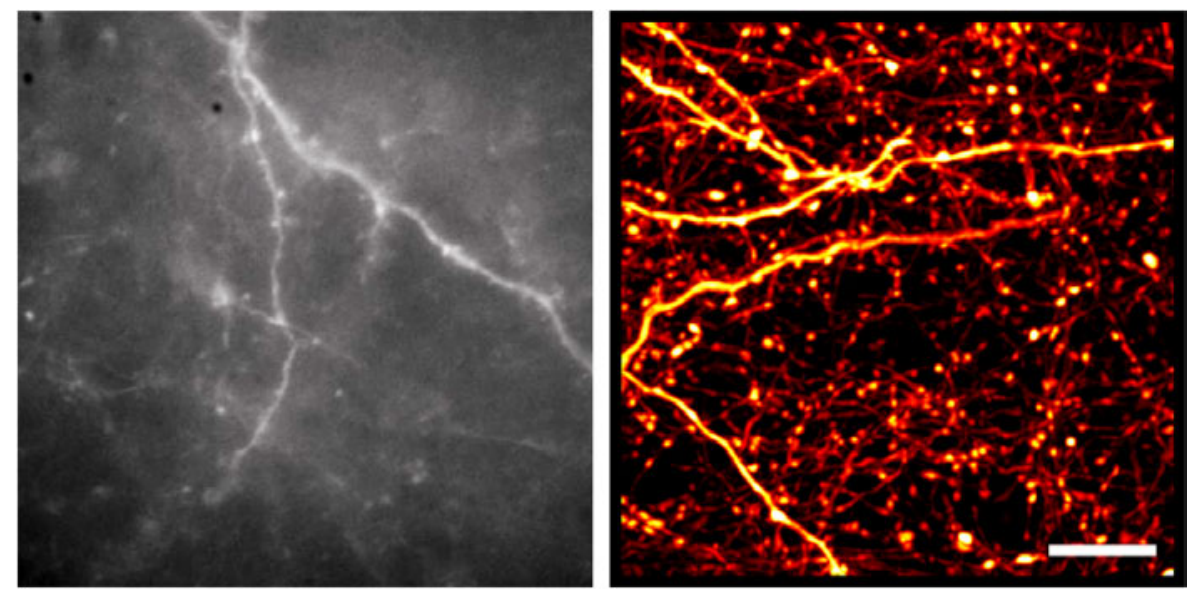

Fig. 4.1: Typical overview of a cortex region of a transgenic mouse prepared for imaging. The left image is observed on a wide-field camera. The right picture is a maximumintensity projection of a confocal overview stack extending over a depth of $5 \mu \mathrm{m}$. Scale bar $=10 \mu \mathrm{m}$. 


\section{Experimental results}

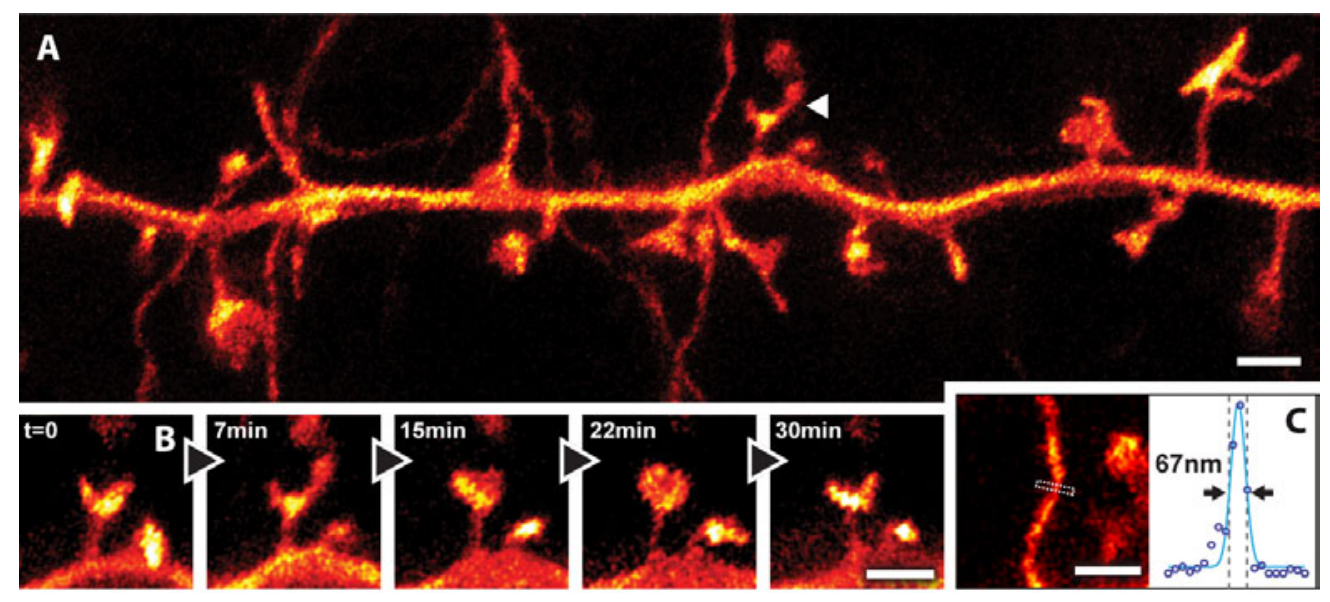

Fig. 4.2: STED microscopy in the molecular layer of the somatosensory cortex of a mouse with EYFP labeled neurons. (A) Projected volumes of dendritic and axonal structures of an anesthetized mouse reveal (B) temporal dynamics of spine morphology with (C) a 4-fold improved resolution compared to diffraction-limited imaging. The curve is a three pixel wide line profile fitted to raw data with a Gaussian. Scale bars $=1 \mu \mathrm{m}$.

relating synaptic and structural plasticity are in large parts still poorly understood and therefore subject to intensive research

In typical experiments, the formation and elimination of entire spines is studied in living brain tissue over time with two-photon microscopy [101]. In addition to these relatively pronounced changes, however, there are also more subtle modifications, which affect the morphology of the spines on the nanoscale. As the spatial resolution of two-photon microscopes usually lies in the range of $250-400 \mathrm{~nm}$, these structural rearrangements within individual spines are hard to quantify. Variations in the diameter of a spine neck, for example, lie in the range of 40 to 500nm [102]. The only way to currently study these small changes is to correlate the two-photon measurements with retrospective electron microscopy reconstructions [103, 104]. Apart from being a time-consuming procedure, this does not allow the study of dynamic morphological features. It would therefore be advantageous if the detailed structure of single spines could be recorded by means of light- instead of electron microscopy. With STED microscopy, movements of dendritic spines in hippocampal organotypical brain slices of newborn mice were observed with greatly enhanced spatial resolution [105]. Furthermore, it was recently shown to be possible to resolve features of the underlying actin cytoskeleton, which is in large parts responsible the structural plasticity of the spines [19]. Until now it has, however, remained unclear whether such movements also occur in the adult brain. Despite previous studies suggesting that spine motility is largely reduced during maturation [95], there are also hints that the remodeling of neuronal circuits in the adult brain could involve such morphological rearrangements at the spine level [94].

The molecular layer of the somatosensory cortex of adult $\operatorname{TgN}$ (Thy 1-EYFP) mice aged $2-12 \mathrm{M}$ was imaged in vivo with the upright STED microscope introduced earlier. The 

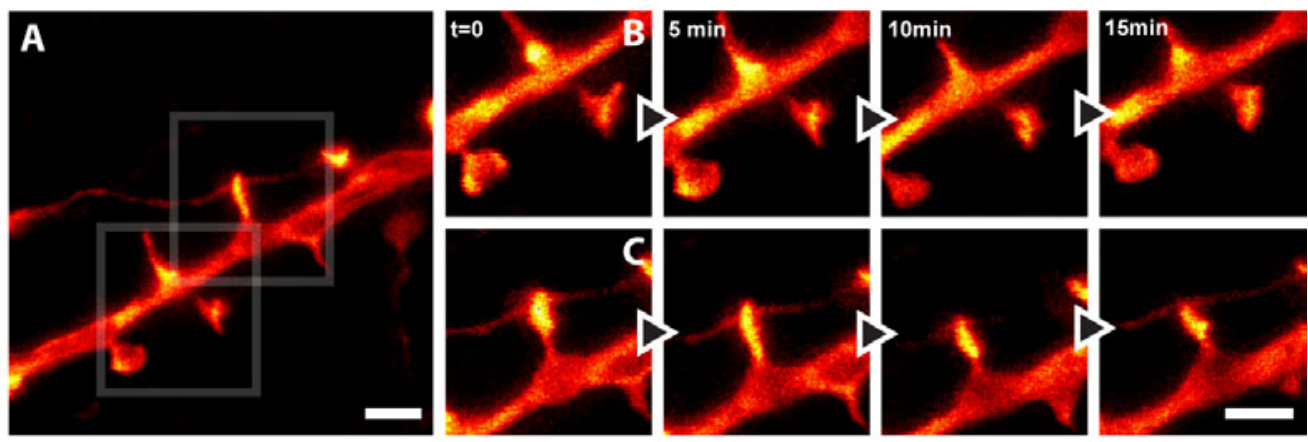

Fig. 4.3: STED recordings of dendritic spines (A) reveal varying levels of spine motility among spines located in close proximity along the same dendrite $(B, C)$. Scale bar $=1 \mu \mathrm{m}$.

mouse strain TgN(Thy1-EYFP) was chosen for the expression of the yellow fluorescent protein (EYFP) in the cytoplasm of a subset of neurons [106, 107]. Fig. 4.1 shows a typical overview of the cortex in wide-field and confocal mode after preparation. Dendritic branches were chosen from the overview to record time-lapse series at high spatial resolution (Figs. 4.2, 4.3). The STED images show structure sizes of $<70 \mathrm{~nm}$ at the finest axons (Fig. 4.2C), indicating that the resolution is at least of that order. Note-worthy are also the negatively contrasted, elongated structures along the dendrite, which could represent mitochondria displacing the stained cytosol (Fig. 4.2). Time-lapse recordings on timescales of several minutes reveal that dendritic spines can indeed undergo continual morphologic changes and movements in the adult brain (Fig. 4.3B). To exclude random defocus from being mistaken for movement, each image was rendered by a maximum projection of a stack of 5 images with $600 \mathrm{~nm}$ depth spacing. While the dendrites of origin retained largely the same shape throughout the experiments, morphological changes were found at the head and neck regions of the dendritic spines, potentially reflecting alterations in the connectivity of the neural network. Motion was observed to be occurring at timescales ranging from a few seconds to minutes and to be of varying magnitude. Variations were thereby not only found between different dendritic branches, but also between closely spaced spines belonging to the same dendrite. A good example can be seen in Fig. 4.3. Even though morphological changes can be seen at the lower two spines of Fig. 4.3B, the filopodia-like spine at the top of the figure and the one depicted in $4.3 \mathrm{C}$ barely move during $15 \mathrm{~min}$. To get a better idea of the timescales involved, small stacks of single spines were recorded with STED at different rates. Fig. 4.4 shows a subset of a time series consisting of 128 projected stacks which were recorded at intervals of 10 seconds over a period of more than 20 minutes. Most changes at the spine head appear continuous at the depicted intervals of one minute, with few exceptions such as the small transient feature appearing only in the third frame (140s). Meanwhile, the small spine located closer to the dendrite does not show any movement.

Even though further quantification of the observed dynamics is beyond the scope of this thesis, it is worthwhile to discuss whether the movements could be induced by the new imaging modality itself. As no other microscopic technique is currently available to resolve features beyond the diffraction limit in the living brain, a definitive answer 

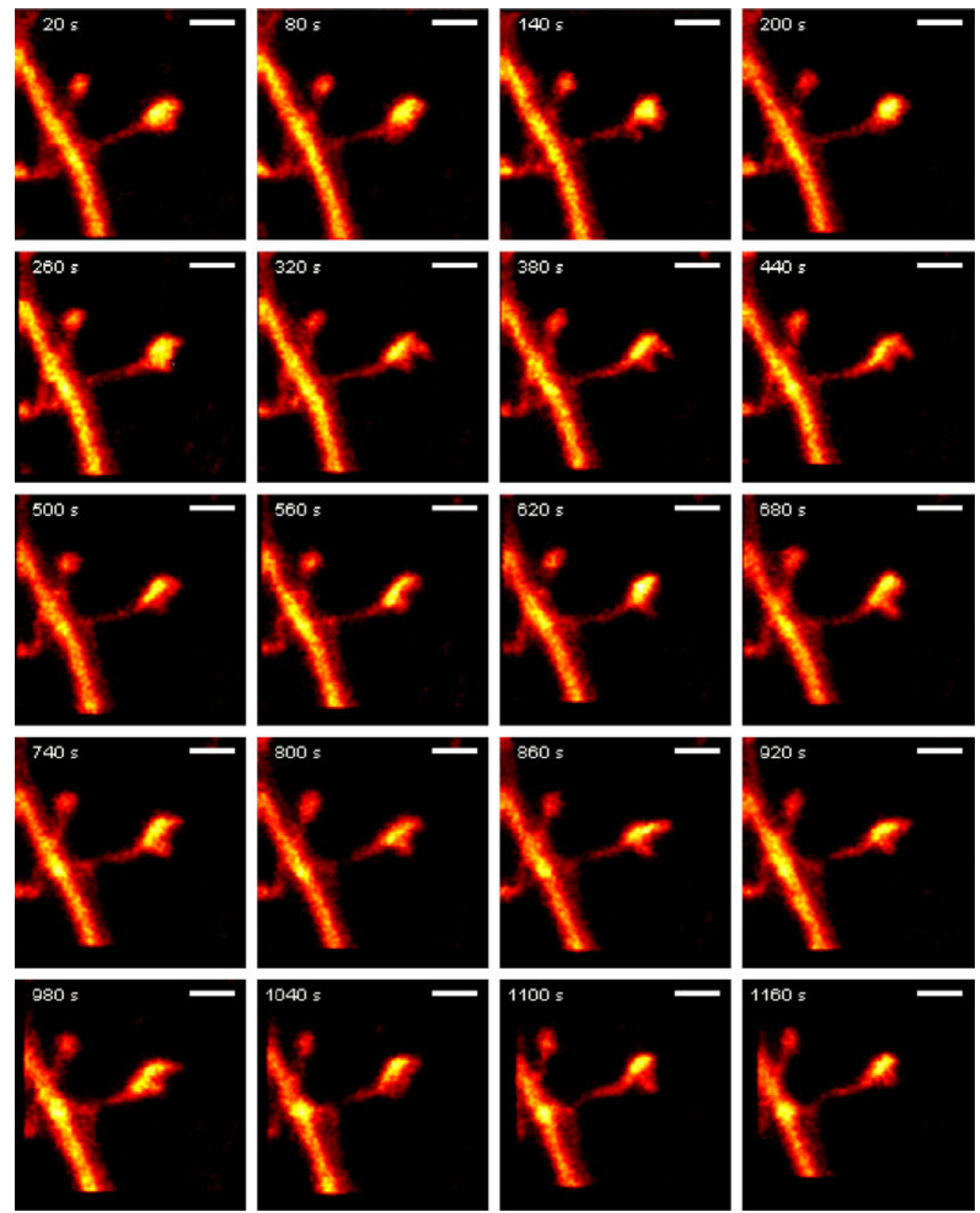

Fig. 4.4: STED time-lapse recording of a single spine at a period of 10 seconds. The measurement includes $128 \mathrm{z}$-stacks consisting of 5 slices each. Showing only one of every six pictures in this overview, most of the rapid remodeling of the spine head still appears continuous and smooth. No damage is observed at the dendrite or the spine after recording a total of 640 slices. Scale bar $=1 \mu \mathrm{m}$. 


\section{Experimental results}

cannot be given at this point. Coarse structural changes at larger spines, however, can readily be detected at confocal resolution. It turns out that timescale and type of the morphological changes observed with STED are not in contradiction with confocal measurements in brain slices reported earlier [108]. For direct comparison, the spine depicted in Fig. 4.4 was imaged every 60 seconds over a timespan of 15 minutes prior to switching on the STED beam (Appendix A.3). Here, also, similar motion can be observed, giving further evidence that STED microscopy does not induce motion beyond the levels seen in confocal microscopy.

Apart from possible functional impairments, interaction of the intense STED light with the tissue could also cause other effects. Although absorption of the $592 \mathrm{~nm}$ STED beam by EYFP is negligible, absorption by intrinsic chromophores in the tissue may occur at this wavelength. Indeed, slight local swellings have occasionally been observed for relatively thick dendritic processes featuring many mitochondria. We did not observe degradations or disaggregations of the processes, however, which have been shown to occur right after cell death or exitus. No evidence was found that STED microscopy introduces more stress to the neurons or to the surrounding tissue compared to established techniques as 2P-microscopy, where similar average focal power levels are commonly employed ${ }^{1}$.

\subsection{Microanatomy of oligodendroglia resolved in vivo}

Apart from neurons, the brain is populated by further groups of cells, one of which comprises neuroglia or simply glial cells. Despite the fact that the neuroglia outnumber the neurons by a factor of ten, they do not actively process information ${ }^{2}$. Instead, they mainly provide supportive functions for neurons. Oligodendroglial cells, also called oligodendrocytes (derived from greek meaning ,cell with few branches“), are among the largest types of glial cells. They are mainly responsible of growing myelin sheaths around axons in the central nervous system (CNS). Myelin, which was already discovered in 1854 by Rudolf Virchow, is a substance consisting mainly of fatty lipids that render it electrically insulating. When a myelin layer is created around an axon, the resistance of the cell membrane is greatly increased and the capacitance reduced. Oligodendrocytes in the CNS usually extend their processes to many different axons. The myelin sheath which is thereby formed around any individual axon, is usually shorter than a few hundred microns. The short discontinuities in the insulation occurring between neighboring myelinated sections are known as nodes of Ranvier. Due to the myelin acting as an insulator, depolarization can only occur at these nodes, which greatly increases the velocity at which action potentials spread along the axon. This mechanism, called salutatory conduction, also significantly reduces the energy required to propagate action potentials over long distances. Myelination is therefore considered to be one of the most important evolutionary achievements of higher vertebrates.

\footnotetext{
${ }^{1}$ Albeit 2P-microscopes usually operate at thousandfold higher peak intensities.

${ }^{2}$ It has been shown in the last decade that some neuroglia are indeed capable of influencing neuronal signaling. This will be further emphasized in the next section.
} 


\section{Experimental results}

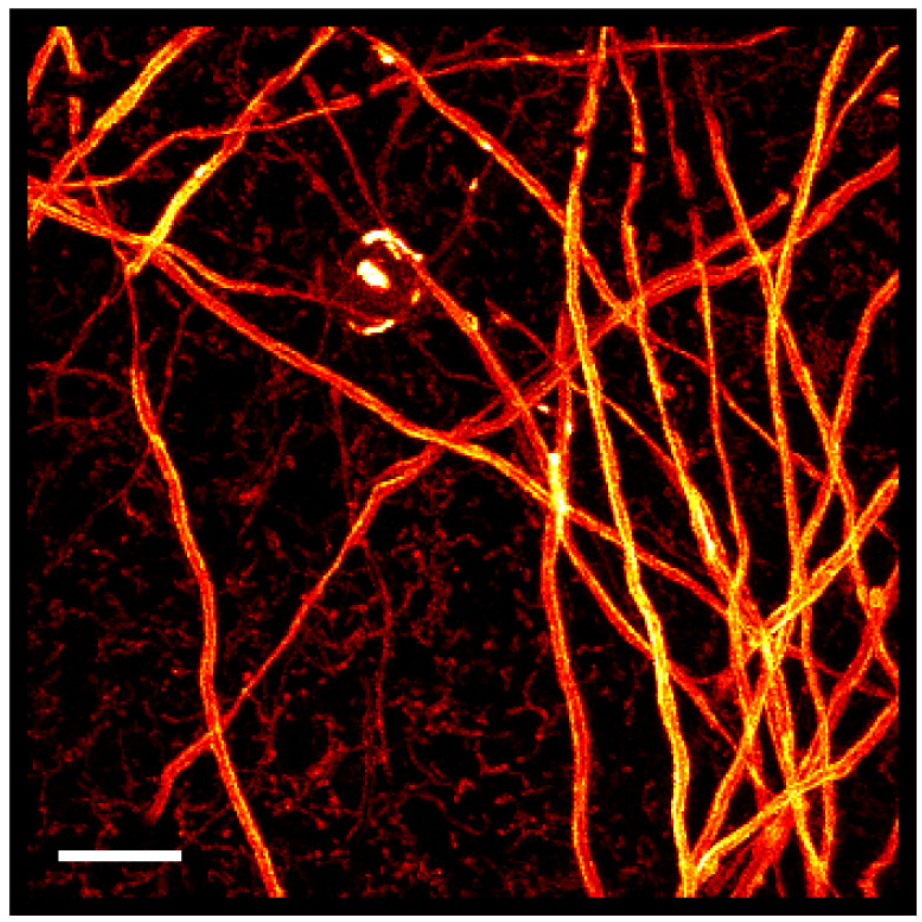

Fig. 4.5: Overview of Oligodendrocytes in the molecular layer of the somatosensory cortex of a transgenic mouse. A cell soma can be observed in the middle of the figure and some of the processes at the top of the frame are interrupted by nodes of Ranvier. Highest expression levels are seen in the myelin sheaths and the endoplasmatic reticulum of the soma. The thin processes leading from the soma to the axons are in contrast much dimmer. Maximum-intensity projection of a confocal stack extending over a depth of $5 \mu \mathrm{m}$. Scale bar $=10 \mu \mathrm{m}$.

The critical role of myelin for the transfer of information in the brain and throughout the nervous system becomes strikingly evident, once it has become degraded in the course of certain neurodegenerative diseases. The most wide-spread among these diseases is certainly multiple sclerosis (MS), which causes demyelination of motory and sensory neurons in the brain and the spinal cord. Patients suffering from MS experiences increasing disability throughout their life, and often have to depend on full-time care less than a decade after the first symptoms have appeared. Little is known, however, about the cause of the disease. Understanding exactly how the myelin sheaths, and thus the oligodendrocytes, are affected and ultimately destroyed during the progression of MS is therefore receiving much attention in medical research $[109,110]$.

The transgenic mouse line PLP-EGFP ${ }^{1}$ used for the experiments in this section expresses the enhanced green fluorescent protein as a reporter of the proteolipid protein (PLP) [111, 112]. PLP accounts for the largest protein fraction in myelin and plays a role in compacting and holding the myelin layers together. Even though it is also expressed in some neurons, PLP is mostly found in myelin-producing cells. It remains to be clarified, however, whether GFP is expressed in all of the oligodendrocytes of

\footnotetext{
${ }^{1}$ Provided by the Max Planck Institute for Experimental Medicine, unpublished
} 

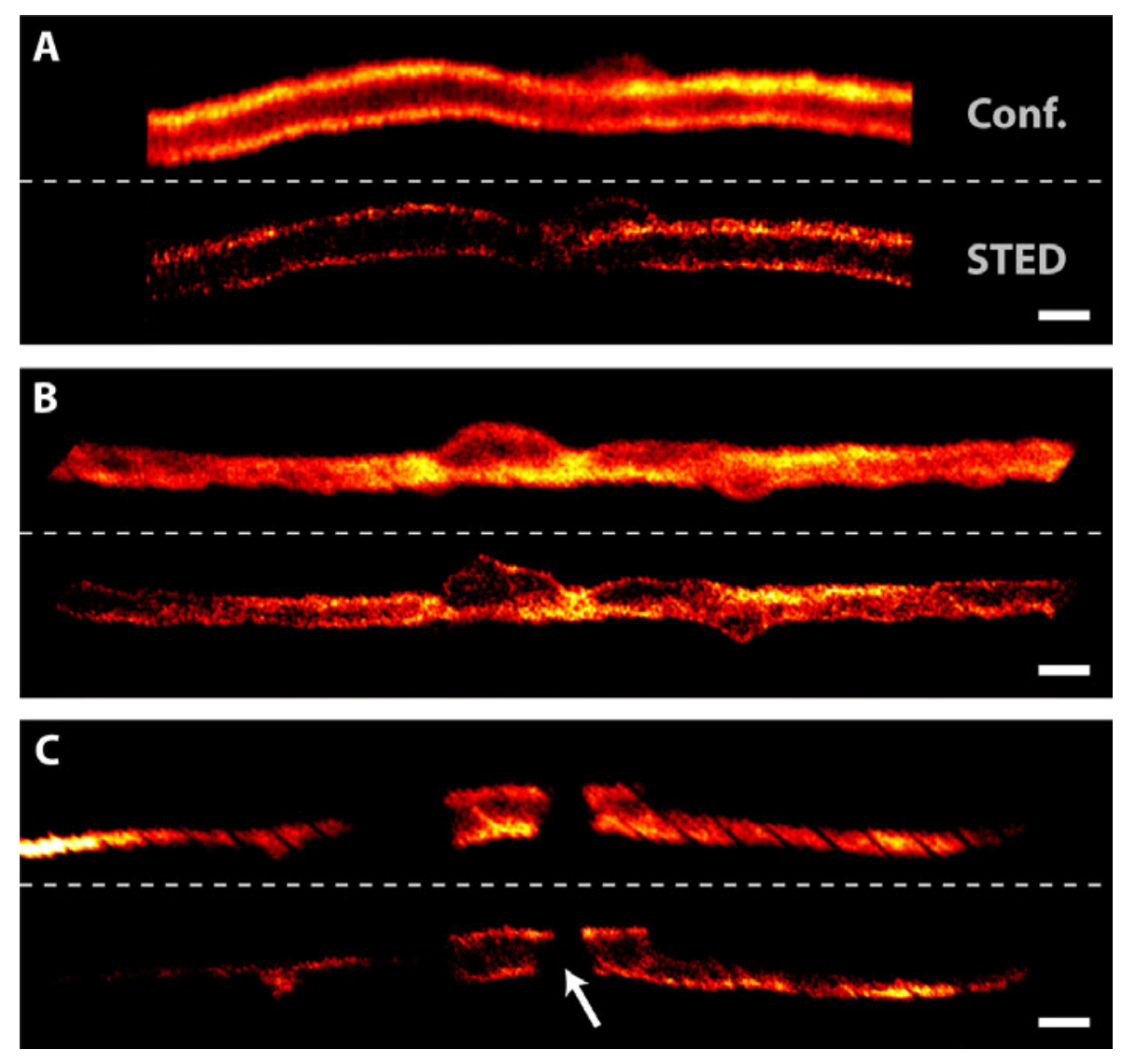

Fig. 4.6: Oligodendroglial cell processes enclosing unstained axons mostly show up as hollow tubes (A). With STED, finer details of the myelin structure can be discerned compared to diffraction-limited confocal recordings. The characteristic pockets in the membrane enclose orthogonally intersecting or branching axons which are being myelinated by the same cell (B). A node of Ranvier (arrow) which is overlaid by respiratory motion artifacts is depicted in (C). Scale bars $=1 \mu \mathrm{m}$.

these mice or only in a subset. The overview in Fig. 4.5 is exemplary for the density of labeled structures seen throughout the measurements.

A selection of typical images obtained of oligodendroglial processes ensheathing thin axons is shown in Fig. 4.6. To demonstrate the resolution enhancement achieved with STED, a confocal recording is juxtaposed to each depicted structure. The labeled myelin sheaths in this region of the cortex appear mostly as hollow tubes with a diameter of approximately $1 \mu \mathrm{m}$ and a thickness of 200-300nm in the diffraction-limited case (Fig. 4.6A). Using STED, the FWHM of the myelin sheaths is reduced to below $100 \mathrm{~nm}$ at many locations and finer details of the inner structure become visible (Fig. 4.6B). The thinnest cross-sections are consistently found to have a diameter of 5070nm (Appendix A.4), most likely reflecting the effective resolution of the microscope in the current configuration. Assuming that most of the labeled proteolipid protein collocates with the compact myelin, the images can be interpreted as a map of the myelin distribution along the axons. The total thickness of the myelin sheath was determined by STED microscopy here to lie in the range of 50-120nm in most areas. With each 

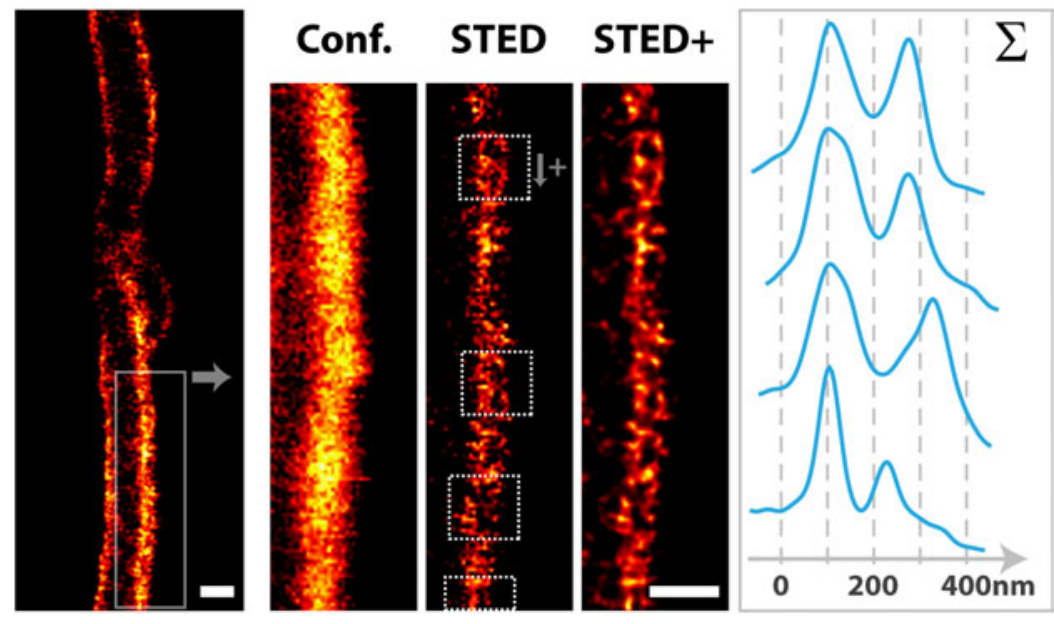

Fig. 4.7: Magnification of the STED image in Fig. 4.6A (repeated on the left) reveals that a subsection of the myelin structure consists of two layers. This is further emphasized by linear deconvolution (STED+). As depicted on the right, the summation over the marked areas clearly shows two peaks, which are separated by $120-210 \mathrm{~nm}$. Scale bars $=500 \mathrm{~nm}$.

myelin layer amounting to $12 \mathrm{~nm}$ [113], this corresponds to roughly 4-8 layers. This is in good agreement with the electronmicroscopic data reported for thin axons in the rat brain [114]. Further evidence for the hypothesis that the structures in the STED images indeed reflect the entire thickness of the myelin sheaths is found at the nodes of Ranvier (Fig. 4.6C). At the node, the structure is abruptly terminated. A sub-structure of the cell, such as the outermost membrane would be expected to contract towards the center of the axon. But there are also some features which cannot immediately be explained. A closer look at Fig. 4.6A shows that the thickness of the right upper section of the myelin sheath differs from that found in rest of the structure. The magnified view in Fig. 4.7 reveals that this section is likely to consist of two myelin stacks located 120-210nm apart. Myelin sheaths measuring roughly twice the average thickness were repeatedly found in the STED images, even if the double-layer structure could not always be resolved. These structures could be caused by folds in the myelin sheath. They are, however, not usually found in electron micrographs.

All of the present knowledge of the sub-cellular structure of the nervous system has been obtained by electron microscopy. As electron microscopy does not allow observing tissue in its natural, hydrated state, the question remains how faithful the structures in the living brain are reproduced by this technique. For features measuring some tens of nanometers, as the thickness of myelin sheaths, STED microscopy could therefore provide a complementary means to verify the microanatomy of neurons and neuroglia. Concluding from the initial findings presented in this section, STED microscopy shows the potential to enable quantitative studies of myelination dynamics in vivo. Chronic experiments with STED could ultimately reveal the long-term progression of restorative myelination following traumata as well as dymyelination processes associated with multiple sclerosis and other neurological disorders. 


\section{Experimental results}

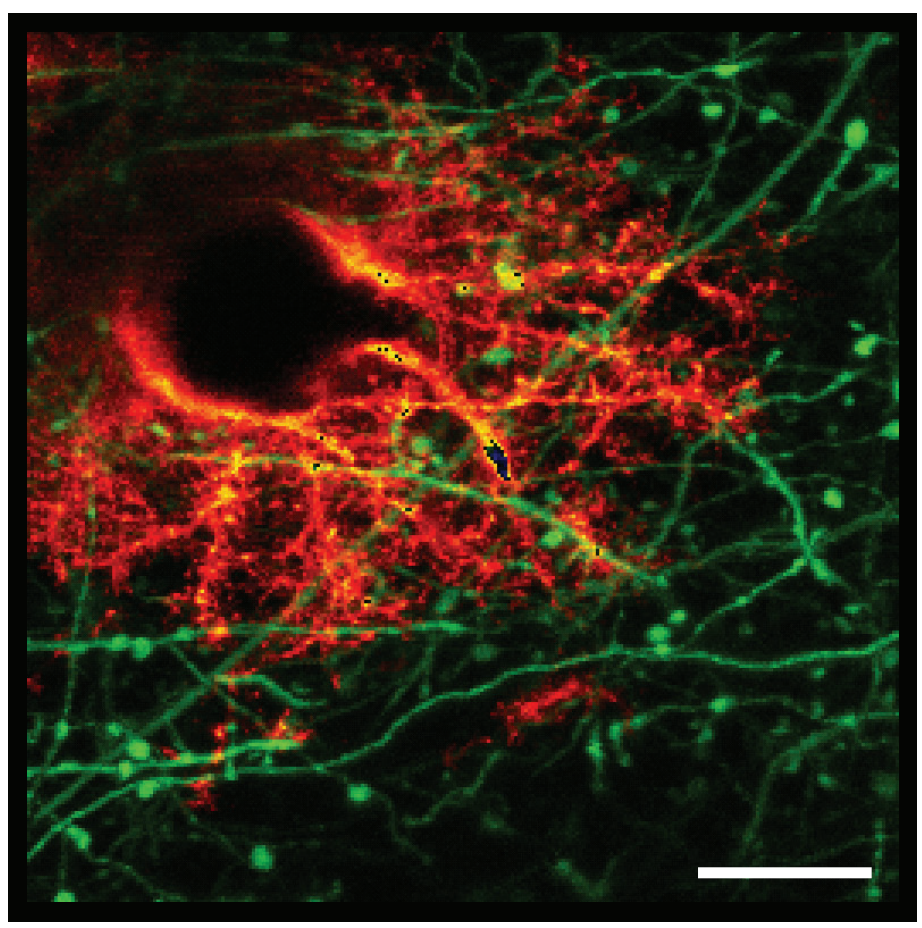

Fig. 4.8: Overview showing a star-shaped protoplasmic astrocyte expressing EGFP (depicted red) surrounded by neurons expressing EYFP (depicted green). The confocal stack was recorded in the molecular layer of the somatosensory cortex of a doubletransgenic mouse. The astrocyte has grown around a small blood vessel, which is quite common as astrocytes form part of the blood-brain barrier. Image stack consists of three slices extending over $2 \mu \mathrm{m}$. Scale bar $=10 \mu \mathrm{m}$.

\subsection{Dual-color STED microscopy of neurons and astrocytes}

Astrocytes (or astroglia) are the neuroglial cells constituting the majority of the glial population in the brain. They provide a wealth of supportive functions to the neurons such as supplying them with nutrients. Additionally, they also control the biochemical environment in the brain by maintaining physiological ion concentrations and by actively removing neurotransmitters from the extracellular space.

In the mid-90s, however, evidence started to emerge that astrocytes are also actively involved in neural signal processing [115]. In the time since it has been shown that bidirectional communication exists between astroglia and neurons ${ }^{1}[117,118]$. Here, communication with the synapse is physically achieved by enwrapping it with a thin glial process. Apart from responding to neural activity and modulating synaptic transmission, astrocytes also exhibit complex intracellular calcium dynamics [119], much like neurons. The importance for synaptic function which is credited to astrocytes today is reflected by the expression "tripartite synapse", adding the perisynaptic glial process as a functional synaptic unit to the pre- and post-synapse [120].

\footnotetext{
${ }^{1}$ Again, Santiago Ramón y Cajal was ahead of his time when he envisioned astrocytes acting as regula-
} tors of synaptic activity in 1897 [116]. 


\section{Experimental results}
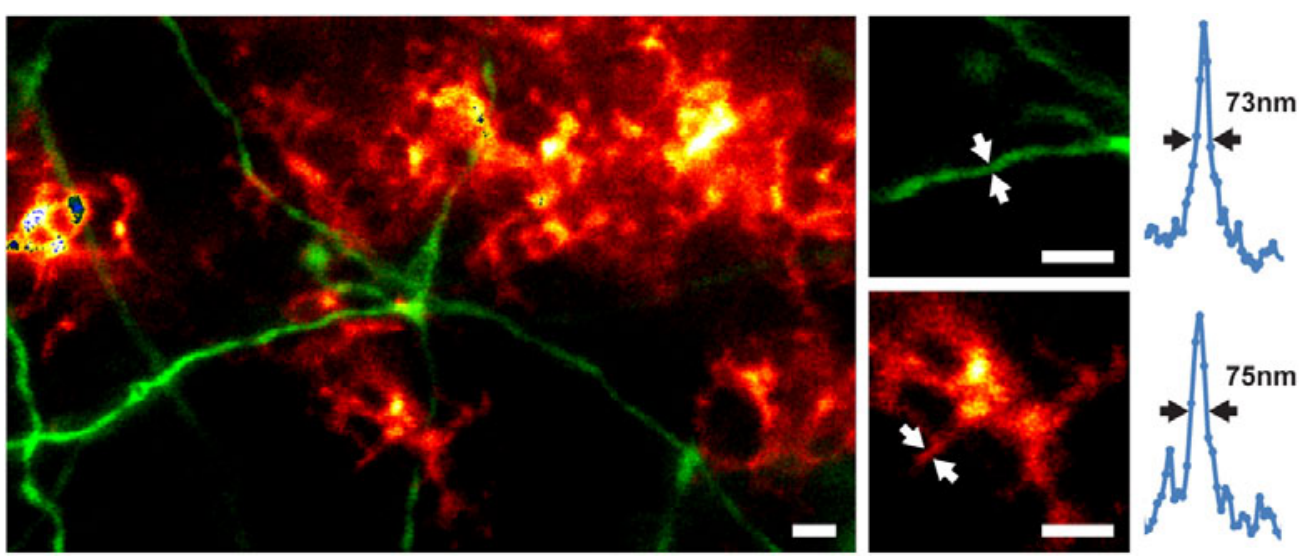

Fig. 4.9: STED microscopy can be used to separate two genetically encoded markers at high spatial resolution in vivo. Small processes of astrocytes (red channel) and neurons (green channel) are clearly resolved beyond the diffraction limit at low channel crosstalk. The images are maximum-intensity projections of two slices recorded at $600 \mathrm{~nm}$ distance. Scale bars $=1 \mu \mathrm{m}$.

To reveal topographic relations between glial- and synaptic processes, one usually has to rely on electron microscopy 3D reconstructions [121, 122], as light microscopes cannot visualize them with sufficient spatial resolution [123]. Nevertheless, it is evident that light microscopy is the only practical way to image structures in intact brain tissue, where dynamics can be observed. This is especially important as it is now known that the morphology of the perisynaptic processes changes, similar to that of dendritic spines discussed earlier, in response to synaptic activity [124, 125]. As a recent review article on the topic points out [126], the spatial resolution of 2P-microscopy is currently insufficient to study astroglial morphology at the synapses in vivo. To achieve the necessary resolution of $<100 \mathrm{~nm}$, the author therefore proposes to employ STED microscopy for future studies.

To demonstrate the feasibility of two-color STED microscopy in vivo, a transgenic mouse model was chosen which jointly expresses EGFP in astrocytes and EYFP in neurons. In addition to the TgN(Thy1-EYFP) transgene, which was already introduced the first section of this chapter, the mouse line expressed a reporter for the glial fibrillary acidic protein (GFAP) through the $\mathrm{TgN}$ (GFAP-EGFP)GFEC mutation described in [127]. Again, STED images were recorded in the molecular layer of the somatosensory cortex. The confocal overview picture in Fig. 4.8 shows that sparse sub-populations of both cell types expressed the respective fluorescent labels. As with all images shown in this section, no post-processing had to be applied to separate the recorded channels. Only the virtual channels I and IV were used (according to Fig. 3.3, Chap. 3), showing a typical cross-talk below $5 \%$ in the raw data. Here, the green channel is mostly depicted with an over-saturated false-color scheme to visually emphasize the contours of the EYFP-labeled structures. These structures can therefore appear broader compared to those in the EGFP channel.

The recording in Fig. 4.9 demonstrates that sub-diffraction spatial resolution can be 


\section{Experimental results}
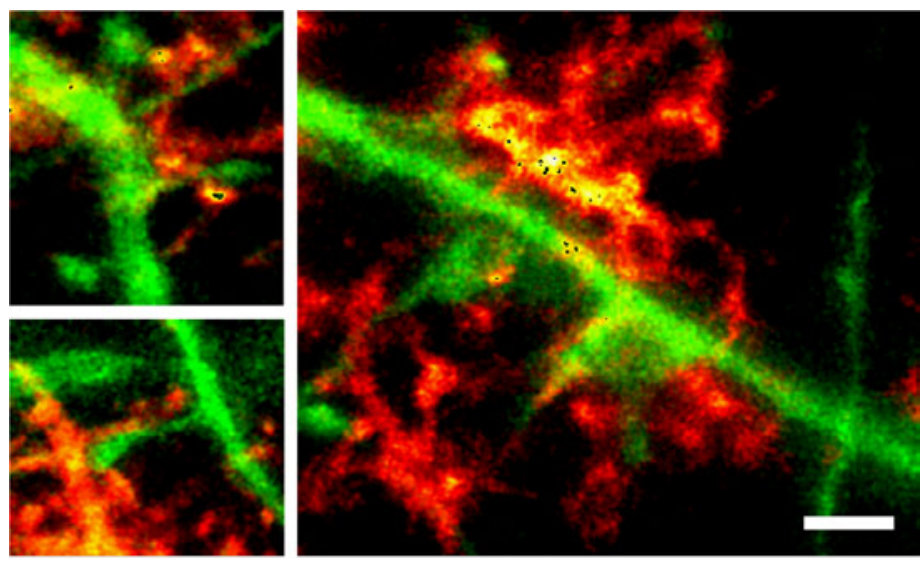

Fig. 4.10: Dual-color STED recordings showing dendritic spines (green channel) and thin processes of astrocytes (red channel) at sub-diffraction resolution. Scale bar $=1 \mu \mathrm{m}$.

achieved in both channels simultaneously. In the case of the astrocytes, single processes can only be resolved at the outer periphery of the cell, as the resolution improvement is limited to the lateral plane in the current configuration. In areas where the coral-like structure of the astroglia is axially extended, the fine substructures are therefore still indiscernible. A selection of STED images depicting dendritic spines in close contact with astroglial processes is shown in Fig. 4.10. The thin necks of the spines and the shapes of the spine heads are clearly resolved in conjunction with subdiffraction structural details of the astrocytes. In order to identify tripartite synapses, however, 3D reconstructions would be indispensable, again requiring an improved resolution in the axial direction.

Dual channel STED microscopy based on two genetically encoded fluorescent proteins was demonstrated for the first time. In vivo dual-color imaging beyond the diffraction limit should therefore be possible in many model animals which are readily expressing these popular markers. The initial results presented here also indicate that STED microscopy could be a valuable technique to further explore the structural interrelation of glia and neurons at the nanoscale in the living brain. 


\section{Conclusion and Outlook}

Even though the exceptional capability of STED microscopy to image living samples at a spatial resolution far beyond the diffraction limit has been known for some time $[19,38]$, it was not evident that the same would be possible in living animals. Imaging cells in their natural context is, however indispensable since the properties of complex systems, such as the brain, can ultimately not be deduced from studies based on cultured cells and tissue samples alone.

In this thesis, it was demonstrated for the first time that diffraction-unlimited imaging is indeed possible in the cortex of anesthetized mice. The reported 4-fold resolution improvement over conventional optical microscopy is on par with that reported for cultured brain slices [105]. Evaluating the state-of-the-art of in vivo microscopy, it becomes clear that in practice usually not even the expected diffraction-limited performance is achieved. A lateral resolution of $400-500 \mathrm{~nm}$ is thereby often considered the standard for in vivo microscopy by many experimentalists [128, 129]. As shown in the previous sections, however, imaging in living animals is not intrinsically limited to inferior results compared to those obtained in cultured samples.

The technical complexity that was necessary to achieve these results was, nevertheless, on a manageable level. The preparatory techniques that were developed to passively stabilize the mouse to a degree that enabled nanometer-scale imaging can be easily adopted by any skilled surgeon. This is in contrast with sophisticated active compensation mechanisms which have been employed before to suppress respiratory and cardiovascular motion. It has also been shown here that STED microscopy is not necessarily more susceptible to optical aberrations compared to other laser-scanning microscopy techniques, despite the slightly more complex illumination scheme. The acceptable optical tolerances were numerically evaluated for the special case of STED microscopy in brain tissue. It turned out that a few simple measures, such as the utilization of a microscope objective equipped with a correction collar, where sufficient to obtain near-optimal imaging conditions.

Responding to a long-standing request of the scientific community, a new method was presented enabling dual-color super-resolution imaging based on standard fluorescent proteins. The use of a common switching PSF for both colors in conjunction with a pulse-interleaved excitation scheme thereby guarantees spatial colocalization of the channels to within a few nanometers. Despite the large overlap of the emission spectra of the two fluorescent markers, an excellent separation of the channels was achieved. Two-color STED microscopy involving EGFP and EYFP therefore has the potential to reveal the morphological interrelation of different cell species as well as the dynamic interaction of different proteins in vivo.

The aim of this methodologically oriented thesis was explicitly not the elaboration of a certain neurological question. Rather, the focus was to evaluate the applicability 


\section{Conclusion and Outlook}

of in vivo STED microscopy as a general tool for a variety of applications involving different cell types and fluorescent markers. Nevertheless, some conclusions can be drawn from the presented observations related to dendritic spines. There is strong evidence now that the morphological remodeling which was reported to occur in cultured organotypic brain slices before, is also present in the cortex of adult, living mice. Following up on these results, the morphological plasticity should be further evaluated in conjunction with the application of external stimuli in future experiments.

Does STED microscopy thus have the potential to become a disruptive tool for neuroscientific research, similar to two-photon microscopy in the beginning of the 90s? From a technological point of view, there are a couple of challenges that will have to be overcome first.

The most pressing one is the issue of penetration depth. All of the presented images in this thesis were recorded in the upmost layer of the cortex at penetration depths below $20 \mu \mathrm{m}$. While this suffices for initial demonstration purpose of the technique, many applications require at least access to the L2/3 layer of the cortex, which translates to depths of $200-300 \mu \mathrm{m}$. This cannot be achieved in the present configuration, where signals were found to drop below usable levels after only $50-70 \mu \mathrm{m}$. This is mainly due to scattering and pronounced tissue absorption in the visible wavelength range. Above $650 \mathrm{~nm}$ the characteristic absorption length increases by a of factor nearly 100 , which is maintained throughout the near-infrared (NIR) up to $1,5 \mu \mathrm{m}$ [130]. The range in between is often referred to as the "physiological window", which is exploited in 2P-microscopy. The idea is thus to improve STED in a similar fashion. It was shown before that two-photon excitation can be used with a STED laser [128, 131]. This results, however, only in a minor improvement, as the visible STED beam now becomes the limiting factor. Although two-photon operation of the inhibition beam was recently demonstrated for a new organic dye [132], the required pulse power levels are prohibitive for in vivo microscopy. In contrast, the ongoing development of new fluorescent proteins in the NIR range, such as iRFP [133], offers a much more promising perspective. In the case where excitation, detection and stimulated emission can be directly performed at wavelengths within the physiological window, the advantage of two-photon methods becomes less pronounced. Nevertheless, combining far-red fluorescent proteins with two-photon excitation and a STED laser above $700 \mathrm{~nm}$ would probably present the best solution to realize deep-tissue super-resolution microscopy at present.

Another challenge is certainly the reduction of the applied optical power. Even though only minor interactions of the STED beam with the brain tissue were observed during the experiments, power levels should be reduced as far as possible to preclude influencing the observed system. Note that this is also directly related to the topic of intrinsic tissue absorption discussed above. In a recent publication, Chen et al. have shown the importance of reducing pixel dwell-times in 2P-microscopy to the sub-microsecond range to avoid damaging neurons [15]. This could be applied to STED microscopy as well using an acousto- or electro-optic beam scanner. Additionally, this would likely result in an increased fluorescence signal due to the effect of dark-state-relaxation [134]. An additional technique was recently introduced that reduces light exposure to a minimum by employing a clever switching scheme for the lasers [135]. In sparsely labeled 


\section{Conclusion and Outlook}

samples, as the ones presented here, more than $90 \%$ of the total light dosage could probably be saved in this way, as the lasers are mostly switched off in dark areas. As it was recently demonstrated [43, 44], RESOLFT microscopy enables further reduction of the total light exposure. The photo-switchable proteins which are used for this method require $10^{6}$ times less light for switching compared to STED. While the UV wavelengths currently employed for switching conflict with the requirement for better tissue penetration at first glance, the low switching intensities bear the potential for two-photon activated switching. In terms of imaging speed, RESOLFT is currently lagging behind by a factor of ten with respect to STED. This is, however, not a fundamental limitation and will either be addressed by the development of proteins with faster switching kinetics or by parallelization of the image acquisition process [136]. It has to be kept in mind, though, that transgenic animals expressing photo-switchable proteins are not yet available. The STED microscope introduced in this work is therefore currently more generally applicable for in vivo super-resolution imaging due to its compatibility with regular fluorescent proteins.

In conclusion, the current limitations of in vivo STED / RESOLFT microscopy are likely to be overcome in the near future, mostly relying on novel proteins and methodological improvements which have already been demonstrated. STED / RESOLFT microscopy therefore has the potential to greatly expand the range of questions that can be addressed directly in the living animal. Santiago Ramón y Cajal, who this all started with, would certainly be delighted. 


\section{Bibliography}

[1] Santiago Ramón y Cajal. Recuerdos de mi vida. Impr. de J. Pueyo, 1923.

[2] Richard Rapport. Nerve Endings: The Discovery of the Synapse. W. W. Norton \& Company, 2005.

[3] A. van Gehuchten. Anatomie du système nerveux de l'Homme. Louvain, 1897.

[4] E. Pannese. The golgi stain: invention, diffusion and impact on neurosciences. J Hist Neurosci, 8(2):132-140, 1999.

[5] S. Grisolía. Ramón y Cajal's contribution to the neurosciences: proceedings of the Symposium "Horizons in Neuroscience", honoring the 100th anniversary of Santiago Ramón y Cajal's research career. Elsevier, 1983.

[6] G. M. Shepherd. Foundations of the neuron doctrine. Oxford University Press, 1991.

[7] G. E. Palade and S. L. Palay. Electron microscope observations of interneuronal and neuromuscular synapses. Anatomical Record, 118:335-336, 1954.

[8] W. Denk, J. H. Strickler, and W. W. Webb. Two-photon laser scanning fluorescence microscopy. Science, 248(4951):73-76, April 1990.

[9] K. Svoboda, W. Denk, D. Kleinfeld, and D. W. Tank. In vivo dendritic calcium dynamics in neocortical pyramidal neurons. Nature, 385(6612):161-165, January 1997.

[10] M. Chalfie, Y. Tu, G. Euskirchen, W. W. Ward, and D. C. Prasher. Green fluorescent protein as a marker for gene expression. Science, 263(5148):802-805, February 1994.

[11] R. Y. Tsien. The green fluorescent protein. Annu. Rev. Biochem., 67:509-544, July 1998.

[12] K. K. Ghosh, L. D. Burns, E. D. Cocker, A. Nimmerjahn, Y. Ziv, and A. El Gamal an M. J Schnitzer. Miniaturized integration of a fluorescence microscope. Nat. Methods, 8(10):871-878, October 2011.

[13] W. Piyawattanametha, E. D. Cocker, L. D. Burns, R. P. J. Barretto, J. C. Jung, H. Ra, O. Solgaard, and M. J. Schnitzer. In vivo brain imaging using a portable $2.9 \mathrm{~g}$ two-photon microscope based on a microelectromechanical systems scanning mirror. Opt. Lett., 34(15):2309-2311, August 2009.

[14] B. F. Grewe, D. Langer, H. Kasper, B. M. Kampa, and F. Helmchen. Highspeed in vivo calcium imaging reveals neuronal network activity with nearmillisecond precision. Nat. Methods, 7(5):399-405, May 2010. 


\section{Bibliography}

[15] X. Chen, U. Leischner, N. L. Rochefort, I. Nelken, and A. Konnerth. Functional mapping of single spines in cortical neurons in vivo. Nature, 475:501-505, July 2011.

[16] W. Mittmann, D. J. Wallace1, U. Czubayko, J. T. Herb, A. T. Schaefer, L. L. Looger, W. Denk, and J. N. D. Kerr. Two-photon calcium imaging of evoked activity from 15 somatosensory neurons in vivo. Nat. Neurosci., 14(8):10891094, August 2011.

[17] P. Theer, M. T. Hasan, and W. Denk. Two-photon imaging to a depth of 1000 microns in living brains by use of a Ti:Al203 regenerative amplifier. Opt. Lett., 28(12):1022-1024, June 2003.

[18] S. W. Hell. Microscopy and its focal switch. Nat. Methods, 6(1):24-32, January 2009.

[19] N. T. Urban, K. I. Willig, S. W. Hell, and U. V. Nägerl. STED nanoscopy of actin dynamics in synapses deep inside living brain slices. Biophys. J., 101: 1277-1284, September 2011.

[20] Y. Hua, R. Sinha, C. S. Thiel, R. Schmidt, J. Hüve, H. Martens, S. W Hell, A. Egner, and J. Klingauf. A readily retrievable pool of synaptic vesicles. Nat. Neurosci., 14(7):833-839, July 2011.

[21] A. Dani, B. Huang, J. Bergan, C. Dulac, and X. Zhuang. Superresolution imaging of chemical synapses in the brain. Neuron, 68(5):843-856, December 2010.

[22] N. A. Frost, H. Shroff, H. Kong, E. Betzig, and T. A. Blanpied. Single-molecule discrimination of discrete perisynaptic and distributed sites of actin filament assembly within dendritic spines. Neuron, 67(1):86-99, July 2010.

[23] A. C. Meyer, T. Frank, D. Khimich, G. Hoch, D. Riedel, N. M. Chapochnikov, Y. M. Yarin, B. Harke, S. W. Hell, A. Egner, and T. Moser. Tuning of synapse number, structure and function in the cochlea. Nat. Neurosci., 12(4):444-453, April 2009.

[24] K. I. Willig, S. O. Rizzoli, V. Westphal, R. Jahn, and S. W. Hell. STEDmicroscopy reveals that synaptotagmin remains clustered after synaptic vesicle exocytosis. Nature, 440(7086):935-939, April 2006.

[25] E. Abbe. Beiträge zur Theorie des Mikroskops und der mikroskopischen Wahrnehmung. Archiv für Mikroskopische Anatomie, 9:413-418, 1873.

[26] O. Heimstädt. Das Fluoreszenzmikroskop. Z. Wiss. Mikrosk., 28:330-337, 1911.

[27] A. H. Coons, H. J. Creech, R. N. Jones, and E. Berliner. The demonstration of pneumococcal antigen in tissues by the use of fluorescent antibody. J. Immunol., 45:159-170, 1942. 


\section{Bibliography}

[28] A. Lewis, H. Taha, A. Strinkovski, A. Manevitch, A. Khatchatouriants, R. Dekhter, and E. Ammann. Near-field optics: from subwavelength illumination to nanometric shadowing. Nat. Biotechnol., 21(11):1378-1386, November 2003.

[29] H. F. Hamann, A. Gallagher, and D. J. Nesbitt. Near-field fluorescence imaging by localized field enhancement near a sharp probe tip. Appl. Phys. Lett., 76(14): 1953-1955, April 2000.

[30] R. M. Stöckle, Y. D. Suh, V. Deckert, and R. Zenobi. Nanoscale chemical analysis by tip-enhanced raman spectroscopy. Chem. Phys. Lett., 318:131-136, February 2000.

[31] S. Takahashi and A. V. Zayats. Near-field second-harmonic generation at a metal tip apex. Appl. Phys. Lett., 80(19):3479-3481, 2002.

[32] Q. Wu, G. D. Feke, R. D. Grober, and L. P. Ghislain. Realization of numerical aperture 2.0 using a gallium phosphide solid immersion lens. Appl. Phys. Lett., 75(26):4064-4066, December 1999.

[33] S. W. Hell and J. Wichmann. Breaking the diffraction resolution limit by stimulated emission. Opt. Lett., 19(11):780-782, June 1994.

[34] A. Einstein. Zur Quantentheorie der Strahlung. Physik. Zeitschr., 18:121-128, 1917.

[35] B. Harke, J. Keller, C. K. Ullal, V. Westphal, A. Schönle, and S. W. Hell. Resolution scaling in STED microscopy. Opt. Express, 16(6):4154 - 4162, March 2008 .

[36] E. Rittweger, K. Young Han, S. E. Irvine, C. Eggeling, and S. W. Hell. STED microscopy reveals crystal colour centres with nanometric resolution. Nat. Photonics, 3:144-147, March 2009.

[37] T. A. Klar, S. Jakobs, M. Dyba, A. Egner, and S. W. Hell. Fluorescence microscopy with diffraction resolution barrier broken by stimulated emission. PNAS, 97(15):8206-8210, July 2000.

[38] B. Hein, K. I. Willig, and S. W. Hell. Stimulated emission depletion (STED) nanoscopy of a fluorescent protein-labeled organelle inside a living cell. PNAS, 105(38):14271-14276, September 2008.

[39] S. W. Hell and M. Kroug. Ground-state depletion fluorescence microscopy, a concept for breaking the diffraction resolution limit. Appl. Phys. B, 60:495-497, 1995.

[40] S. Bretschneider, C. Eggeling, and S. W. Hell. Breaking the diffraction barrier in fluorescence microscopy by optical shelving. Phys. Rev. Lett., 98(21):218103, May 2007.

[41] M. Schwentker, H. Bock, M. Hofmann, S. Jakobs, J. Bewersdorf, C. Eggeling, and S. W. Hell. Wide-field subdiffraction RESOLFT microscopy using fluorescent protein photoswitching. Micr. Res. Tech., 70(3):269-280, 2007. 


\section{Bibliography}

[42] M. Hofmann, C. Eggeling, S. Jakobs, and S.W. Hell. Breaking the diffraction barrier in fluorescence microscopy at low light intensities by using reversibly photoswitchable proteins. PNAS, 102(49):17565-17569, 2005.

[43] T. Grotjohann, I. Testa, M. Leutenegger, H. Bock, N. T. Urban, F. LavoieCardinal, K. I.Willig, C. Eggeling, S. Jakobs, and S. W. Hell. Diffractionunlimited all-optical imaging and writing with a photochromic GFP. Nature, 478(7368):204-208, October 2011.

[44] T. Brakemann, A. C. Stiel, G. Weber, M. Andresen, I. Testa, T. Grotjohann, M. Leutenegger, U. Plessmann, H. Urlaub, C. Eggeling, M. C. Wahl, S. W. Hell, and S. Jakobs. A reversibly photoswitchable GFP-like protein with fluorescence excitation decoupled from switching. Nat. Biotechnol., 29(10):942-947, October 2011.

[45] E. Betzig, G. H. Patterson, R. Sougrat, O. W. Lindwasser, S. Olenych, J. S. Bonifacino, M. W. Davidson, J. Lippincott-Schwartz, and H. F. Hess. Imaging intracellular fluorescent proteins at nanometer resolution. Science, 313(5793): 1642-1645, September 2006.

[46] M. J. Rust, M. Bates, and W. Zhuang. Sub-diffraction-limit imaging by stochastic optical reconstruction microscopy (STORM). Nat. Methods, 3(10):793-796, October 2006.

[47] J. Fölling, M. Bossi, H. Bock, R. Medda, C. A. Wurm, B. Hein, S. Jakobs, C. Eggeling, and S. W. Hell. Fluorescence nanoscopy by ground-state depletion and single-molecule return. Nat. Methods, 5(11):943-945, November 2008.

[48] B. Huang, W. Wang, M. Bates, and X. Zhuang. Three-dimensional superresolution imaging by stochastic optical reconstruction microscopy. Science, 319(5864):810-813, January 2008.

[49] S. R. P. Pavani, M. A. Thompson, J. S. Biteen, S. J. Lord, N. Liu, R. J. Twieg, R. Piestun, and W. E. Moerner. Three-dimensional single-molecule fluorescence imaging beyond the diffraction limit using a double-helix point spread function. PNAS, 106(9):2995-2999, March 2009.

[50] P. A. Muriello and K. W. Dunn. Improving signal levels in intravital multiphoton microscopy using an objective correction collar. Optical Commun., 281(7): 1806-1812, April 2008.

[51] A. Egner and S. W. Hell. Handbook of Biological Confocal Microscopy, chapter 20, pages 404-413. Springer, 2006.

[52] C-Y. Dong, B. Yu, P. D. Kaplan, and P. T.C. So. Performances of high numerical aperture water and oil immersion objective in deep-tissue, multi-photon microscopic imaging of excised human skin. Micr. Res. Tech., 63:81-86, 2004.

[53] L. Sherman, J. Y. Ye, O. Albert, and T. B. Norris. Adaptive correction of depthinduced aberrations in multiphoton scanning microscopy using a deformable mirror. J Microsc, 206(1):65-71, April 2002. 


\section{Bibliography}

[54] M. Booth and T. Wilson. Refractive-index-mismatch induced aberrations in single-photon and two-photon microscopy and the use of aberration correction. J. Biomed. Opt., 6(3):266-272, July 2001.

[55] P. Török, P. Varga, Z. Laczik, and G. R. Booker. Electromagnetic diffraction of light focused through a planar interface between meterials of mismatched refractive indices: an integral representation. J. Opt. Soc. Am. A, 12(2):325332, February 1995.

[56] H. Jacobsen, P. Hänninen, E. Soini, and S. W. Hell. Refractive-index-induced aberrations in two-photon confocal reflectance microscopy. J Microsc, 176(3): 226-230, December 1994.

[57] S. W. Hell, G. Reiner, C. Cremer, and E. H. K. Stelzer. Aberrations in confocal fluorescence microscopy induced by mismatches in refractive index. J Microsc, 169:391-405, March 1993.

[58] S. Deng, L. Liu, Y. Cheng, R. Li, and Z. Xu. Effects of primary aberrations on the fluorescence depletion patterns of STED microscopy. Opt. Express, 18(2): 1657-1666, January 2010.

[59] S. Deng, L. Liu, Y. Cheng, R. Li, and Z. Xu. Investigation of the influence of the aberration induced by a plane interface on STED microscopy. Opt. Express, 17(3):1714-1724, February 2009.

[60] P. Török and P. R. T. Munro. The use of gauss-laguerre vector beams in STED microscopy. Opt. Express, 12(15):3605-3617, July 2004.

[61] B. Richards and E. Wolf. Electromagnetic diffraction in optical systems II.Structure of the image field in an aplanatic system. Proc. R. Soc. A, 253: 358-379, 1959.

[62] P. Debye. Das Verhalten von Lichtwellen in der Nähe eines Brennpunktes oder einer Brennlinie. Ann. Phys., 335(14):755-776, 1909.

[63] B. Hein, K. I. Willig, C. A. Wurm, V. Westphal, S. Jakobs, and S. W. Hell. Stimulated emission depletion nanoscopy of living cells using SNAP-tag fusion proteins. Biophys. J., 98:158-163, January 2010.

[64] B. R. Rankin, G. Moneron, C. A. Wurm, J. C. Nelson, A. Walter, D. Schwarzer, J. Schroeder, D. A. Colón-Ramos, and S. W. Hell. Nanoscopy in a living multicellular organism expressing GFP. Biophys. J., 100:L63 - L65, June 2011.

[65] A. Egner and S. W. Hell. Equivalence of the huygens-fresnel and debye approach for the calculation of high aperture point-spread functions in the presence of refractive index mismatch. J Microsc, 193:244-249, March 1999.

[66] J. Keller, A. Schönle, and S. W. Hell. Efficient fluorescence inhibition patterns for RESOLFT microscopy. Opt. Express, 15(6):3361-3371, March 2007.

[67] N. Lue, J. Bewersdorf, M. D. Lessard, K. Badizadegan, R. R. Dasari, M. S. Feld, and G. Popescu. Tissue refractometry using hilbert phase microscopy. Opt. Lett., 32(24):3522-3524, December 2007. 


\section{Bibliography}

[68] T. K. Biwas and A. K. Gupta. Retrieval of true color of the internal organ of CT images and attempt to tissue characterization by refractive index: Initial experience. Indian J Radiol Imaging, 12(2):169-178, 2002.

[69] J. Binding, J. B. Arous, J.-F. Léger, S. Gigan, C. Boccara, and L. Bourdieu. Brain refractive index measured in vivo with high-NA defocus-corrected fullfield OCT and consequences for two-photon microscopy. Opt. Express, 19(6): 4833-4847, February 2011.

[70] S. N. Roper, M. D. Moores amd G. V. Gelikonov, F. I. Feldchtein, N. M. Beach, M. A. King, V. M. Gelikonov, A. M. Sergeev, and D. H. Reitze. In vivo detection of experimentally induced cortical dysgenesis in the adult rat neocortex using optical coherence tomography. J. Neurosci. Methods, 80:91-98, 1998.

[71] F. A. Duck. Physical Properties of Tissue: A Comprehensive Reference Book. Academic Press Inc., San Diego, 1990.

[72] K. I. Willig, B. Harke, R. Medda, and S. W. Hell. STED microscopy with continuous wave beams. Nat. Methods, 4(11):915-918, 2007.

[73] B. Harke. 3D STED Microscopy with Pulsed and Continuous Wave Lasers. PhD thesis, Univ. of Göttingen, February 2008.

[74] P. Török and P. Varga. Electromagnetic diffraction of light focused through a stratified medium. Appl. Optics, 36(11):2305-2312, April 1997.

[75] R. Arimoto and J. M. Murray. A common aberration with water-immersion objective lenses. J Microsc, 216:49-51, October 2004.

[76] S. Kashima and A. Kashima. Cover slip for use in a microscope, April 1995.

[77] G. Donnert, J. Keller, C. A. Wurm, S. O. Rizzoli, V. Westphal, Andreas Schönle, R. Jahn, S. Jakobs, C. Eggeling, and S. W. Hell. Two-color far-field fluorescence nanoscopy. Biophys. J., 92(8):L67-L69, April 2007.

[78] L. Meyer, D. Wildanger, R. Medda, A. Punge, S. O. Rizzoli, G. Donnert, and S. W. Hell. Dual-color STED microscopy at 30-nm focal-plane resolution. Small, 4(8):1095-1100, 2008.

[79] J. Bückers, D. Wildanger, G. Vicidomini, L. Kastrup, and S. W. Hell. Simultaneous multi-lifetime multi-color STED imaging for colocalization analyses. Opt. Express, 19(4):3130 - 3143, February 2011.

[80] K. I. Willig, A. C. Stiel, T. Brakemann, S. Jakobs, and S. W. Hell. Dual-label STED nanoscopy of living cells using photochromism. Nano Lett., 11:39703973, July 2011.

[81] R. Schmidt, C. A. Wurm, S. Jakobs, J. Engelhardt, A. Egner, and S. W Hell. Spherical nanosized focal spot unravels the interior of cells. Nat. Methods, 5(6): 539-544, June 2008.

[82] B. K. Müller, E. Zaychikov, C. Bräuchle, and D. C. Lamb. Pulsed interleaved excitation. Biophys. J., 89(5):3508 - 3522, August 2005. 


\section{Bibliography}

[83] D. S. Greenberg and J. N. D. Kerr. Automated correction of fast motion artifacts for two-photon imaging of awake animals. J. Neurosci. Methods, 176(1):1-15, January 2009.

[84] S. Landi, E. Putignano, E. M. Boggio, M. Giustetto, T. Pizzorusso, and G. M. Ratto. The short-time structural plasticity of dendritic spines is altered in a model of rett syndrome. Scientific Reports, 1:45, July 2011.

[85] S. Laffray, S. Pagès1, H. Dufour, P. De Koninck, Y. De Koninck, and D. Côté. Adaptive movement compensation for in vivo imaging of fast cellular dynamics within a moving tissue. PLOS ONE, 6(5):e19928, May 2011.

[86] R. Drdla, M. Gassner, E. Gingl, and J. Sandkühler. Induction of synaptic longterm potentiation after opioid withdrawal. Science, 325(5937):207-210, July 2009.

[87] G. Paxinos and K. B. J. Franklin. The mouse brain in stereotaxic coordinates, $2^{\text {nd }}$ Edition. Academic Press, 2001.

[88] M. R. Looney, E. E. Thornton, D. Sen, W. J. Lamm, R. W. Glenny, and M. F. Krummel. Stabilized imaging of immune surveillance in the mouse lung. Nat. Methods, 8(1):91-96, January 2011.

[89] R. C. Thomas. Ion-sensitive intracellular microelectrodes: how to make and use them. Academic Press, 1978.

[90] M. J. O'Donnell. A simple method for construction of flexible, subminiature ion-selective electrodes. J. Exp. Biol., 162:353-359, 1992.

[91] T. Bonhoeffer and R. Yuste. Spine motility: Phenomenology, mechanisms, and function. Neuron, 35:1019-1027, September 2002.

[92] Santiago Ramón y Cajal. Textura del Sistema Nervioso del Hombre y de los Vertebrados. Moya, Madrid, 1899.

[93] H. B. Kwon and B. L. Sabatini. Glutamate induces de novo growth of functional spines in developing cortex. Nature, 474(7349):100-104, June 2011.

[94] V. A. Alvarez and B. L. Sabatini. Anatomical and physiological plasticity of dendritic spines. Annu. Rev. Neurosci., 30:79-97, 2007.

[95] A. J. G. D. Holtmaat, J. T. Trachtenberg, L. Wilbrecht, G. M. Shepherd, X. Zhang, G. W. Knott, and K. Svoboda. Transient and persistent dendritic spines in the neocortex in vivo. Neuron, 45:279-291, January 2005.

[96] M. Maletic-Savatic, R. Malinow, and K. Svoboda. Rapid dendritic morphogenesis in CA1 hippocampal dendrites induced by synaptic activity. Science, 283: 1923-1927, March 1999.

[97] F. Engert and T. Bonhoeffer. Dendritic spine changes associated with hippocampal long-term synaptic plasticity. Nature, 399:66-70, May 1999. 


\section{Bibliography}

[98] T. F. Roberts, K. A. Tschida, M. E. Klein, and R. Mooney. Rapid spine stabilization and synaptic enhancement at the onset of behavioural learning. Nature, 463:948-952, February 2010.

[99] G. Yang, F. Pan, and W.-B. Gan. Stably maintained dendritic spines are associated with lifelong memories. Nature, 462:920-924, December 2009.

[100] Y. Zuo, G. Yang, E. Kwon, and W-B. Gan. Long-term sensory deprivation prevents dendritic spine loss in primary somatosensory cortex. Nature, 436 (7048):261-265, 2005.

[101] D. H. Bhatt, S. Zhang, and W-B. Gan. Dendritic spine dynamics. Annu. Rev. Physiol., 71:261-282, 2009.

[102] K. M. Harris and S. B. Kater. Dendritic spines - cellular specializations imparting both stability and flexibility to synaptic function. Annu. Rev. Neurosci., 17: 341-371, 1994.

[103] G. W. Knott, A. Holtmaat, L. Wilbrecht, E. Welker, and K. Svoboda. Spine growth precedes synapse formation in the adult neocortex in vivo. Nat. Neurosci., 9(9):1117-1124, September 2006.

[104] A. Holtmaat, L. Wilbrecht, G. W. Knott, E. Welker, and K. Svoboda. Experience-dependent and cell-type-specific spine growth in the neocortex. $\mathrm{Na}$ ture, 441:979-983, June 2006.

[105] U. V. Nägerl, K. I. Willig, B. Hein, S. W. Hell, and T. Bonhoeffer. Livecell imaging of dendritic spines by STED microscopy. PNAS, 105(48):1898218987, December 2008.

[106] G. Feng, R. H. Mellor, M. Bernstein, C. Keller-Peck, Q. T. Nguyen, M. Wallace, J. M. Nerbonne, J. W. Lichtman, and J. R. Sanes. Imaging neuronal subsets in transgenic mice expressing multiple spectral variants of GFP. Neuron, 28:4151 , October 2000.

[107] P. G. Hirrlinger, A. Scheller, C. Braun, M. Quintela-Schneider, B. Fuss, J. Hirrlinger, and F. Kirchhoff. Expression of reef coral fluorescent proteins in the central nervous system of transgenic mice. Mol. Cell. Neurosci., 30(3): 291-303, November 2005.

[108] M. E. Dailey and S. J. Smith. The dynamics of dendritic structure in developing hippocampal slices. J Neurosci, 16(9):2983-2994, May 1996.

[109] S. P.J. Fancya, M. R. Kotterb, E. P. Harringtona, J. K. Huangc, C. Zhaoc, D. H. Rowitcha, and R. J. M. Franklin. Overcoming remyelination failure in multiple sclerosis and other myelin disorders. Experimental Neurology, 225(1):18-23, September 2010.

[110] E. M. Frohman, M. K. Racke, and C. S. Raine. Multiple sclerosis - the plaque and its pathogenesis. N. Engl. J. Med., 354:942-955, March 2006. 


\section{Bibliography}

[111] D. Fulton, P. Paez, V. Spreur, V. Handley, C. S. Colwell, A. Campagnoni, and R. Fisher. Developmental activation of the proteolipid protein promoter transgene in neuronal and oligodendroglial cells of neostriatum in mice. Developmental Neuroscience, 33:170-184, 2011.

[112] D. Delaunay, K. Heydon, A. Cumano, M. H. Schwab, J-L. Thomas, U. Suter, K-A. Nave, B. Zalc, and N. Spassky. Early neuronal and glial fate restriction of embryonic neural stem cells. J Neurosci, 28(10):2551-2562, March 2008.

[113] N. Baumann and D. Pham-Dinh. Biology of oligodendrocyte and myelin in the mammalian central nervous system. Physiol. Rev., 81(2):871-927, April 2001.

[114] C. Bjartmar, C. Hildebrand, and K. Lolnder. Morphological heterogeneity of rat oligodendrocy tes: Electron microscopic studies on serial sections. Glia, 11: 235-244, 1994.

[115] V. Parpura, T. A. Basarsky, F. Liu, K. Jeftinija, S. Jeftinija, and P. G. Haydon. Glutamate-mediated astrocyte-neuron signalling. Nature, 369:744-747, June 1994.

[116] V. García-Marín, P. García-López, and M. Freire. Cajal's contributions to glia research. Trends Neurosci., 30(9):479-487, September 2007.

[117] M. M. Halassa and P. G. Haydon. Integrated brain circuits: Astrocytic networks modulate neuronal activity and behavior. Annu. Rev. Physiol., 72:335355, March 2010.

[118] G. Perea, M. Navarrete, and A. Araque. Tripartite synapses: astrocytes process and control synaptic information. Trends Neurosci., 32(8):421-431, July 2009.

[119] M. A. Di Castro, J. Chuquet, N. Liaudet, K. Bhaukaurally, M. Santello, D. Bouvier, P. Tiret, and A. Volterra. Local $\mathrm{ca}^{2+}$ detection and modulation of synaptic release by astrocytes. Nat. Neurosci., 14(10):1276-1283, October 2011.

[120] A. Araque, V. Parpura, R.P. Sanzgiri, and P.G. Haydon. Tripartite synapses: glia, the unacknowledged partner. Trends Neurosci., 22:208-215, 1999.

[121] R. Ventura and K. M. Harris. Three-dimensional relationships between hippocampal synapses and astrocytes. J Neurosci, 19(16):6897-6906, 1999.

[122] J. Grosche, V. Matyash, T. Möller, A. Verkhratsky, A. Reichenbach, and H. Kettenmann. Microdomains for neuron-glia interaction: parallel fiber signaling to bergmann glial cells. Nat. Neurosci., 2(2):139-143, February 1999.

[123] H. Nishida and S. Okabe. Direct astrocytic contacts regulate local maturation of dendritic spines. J Neurosci, 27(2):331-340, 2007.

[124] M. Haber, L. Zhou, and K. K. Murai. Cooperative astrocyte and dendritic spine dynamics at hippocampal excitatory synapses. J Neurosci, 30:8881-8891, 2006.

[125] J. J. Lippman, T. Lordkipanidze, M. E. Buell, S. O. Yoon, and A. Dunaevsky. Morphogenesis and regulation of bergmann glial processes during purkinje cell dendritic spine ensheathment and synaptogenesis. Glia, 56(13):1463-1477, October 2008. 


\section{Bibliography}

[126] A. Reichenbach, A. Derouiche, and F. Kirchhoff. Morphology and dynamics of perisynaptic glia. Brain Res. Rev., 1-2:11-25, May 2010.

[127] C. Nolte, M. Matyash, T. Pivneva, C. G. Schipke, C. Ohlemeyer, U.-K. Hanisch, F. Kirchhoff, and H. Kettenmann. GFAP promoter-controlled EGFP-expressing transgenic mice: A tool to visualize astrocytes and astrogliosis in living brain tissue. Glia, 33(1):72-86, January 2001.

[128] J. B. Ding, K. T. Takasaki, and B. L. Sabatini. Supraresolution imaging in brain slices using stimulated-emission depletion two-photon laser scanning microscopy. Neuron, 63(4):429-437, August 2009.

[129] P. J. Drew, A. Y. Shih, J. D. Driscoll, P. M. Knutsen, P. Blinder, D. Davalos, K. Akassoglou, P. S. Tsai, and D. Kleinfeld. Chronic optical access through a polished and reinforced thinned skull. Nat. Methods, 7(12):981-984, December 2010 .

[130] J. Lecoq and M. J. Schnitzer. An infrared fluorescent protein for deeper imaging. Nat. Biotechnol., 29(8):715-716, August 2011.

[131] G. Moneron and S. W. Hell. Two-photon excitation STED microscopy. Opt. Express, 17(17):14567-14573, November 2009.

[132] K. D. Belfield, M. V. Bondar, A. R. Morales, L. A. Padilha, O. V. Przhonska, and $\mathrm{X}$. Wang. Two-photon sted spectral determination for a new v-shaped organic fluorescent probe with efficient two-photon absorption. ChemPhysChem, 12 (15):2755-2762, October 2011.

[133] G. S. Filonov, K. D. Piatkevich, L-M. Ting, J. Zhang, K. Kim, and V. V. Verkhusha. Bright and stable near-infrared fluorescent protein for in vivo imaging. Nat. Biotechnol., 29(8):757-761, August 2011.

[134] G. Donnert, C. Eggeling, and S. W. Hell. Major signal increase in fluorescence microscopy through dark state relaxation. Nat. Methods, 4(1):81-86, January 2007.

[135] T. Staudt, A. Engler, E. Rittweger, B. Harke, J. Engelhardt, and S. W. Hell. Farfield optical nanoscopy with reduced number of state transition cycles. Opt. Express, 19(6):5644-5657, March 2011.

[136] P. Bingen, M. Reuss, J. Engelhardt, and Stefan W. Hell. Parallelized STED fluorescence nanoscopy. Opt. Express, 19(24):23716-23726, November 2011. 


\section{Appendix}

\section{A.1 Importance of preparation technique}

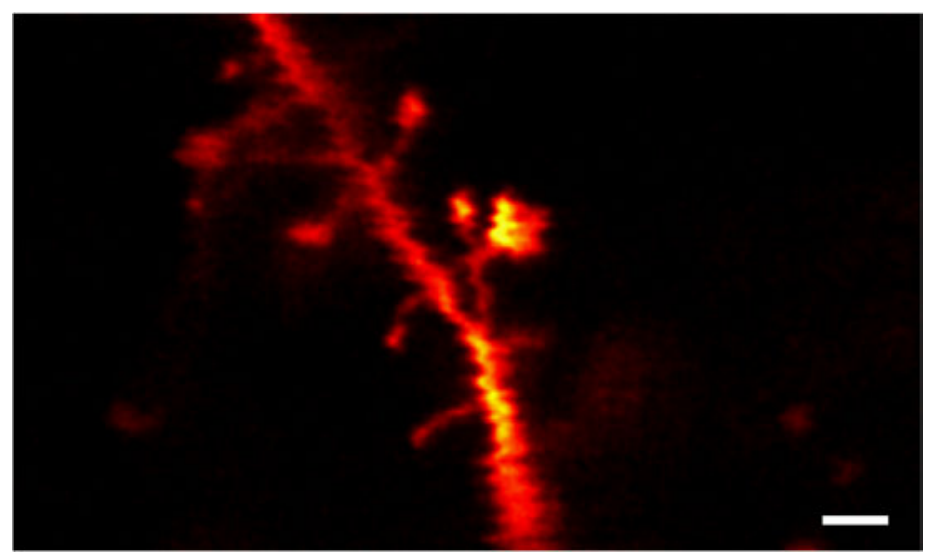

Fig. A.1.1: If the coverslip implantation is not carried out properly, artifacts from respiratory and cardiovascular motion can occur. Here, no drainage was applied and a layer of liquid has formed under the coverslip. Scale bar $=1 \mu \mathrm{m}$. 


\section{A.2 Pictures of the STED microscope}

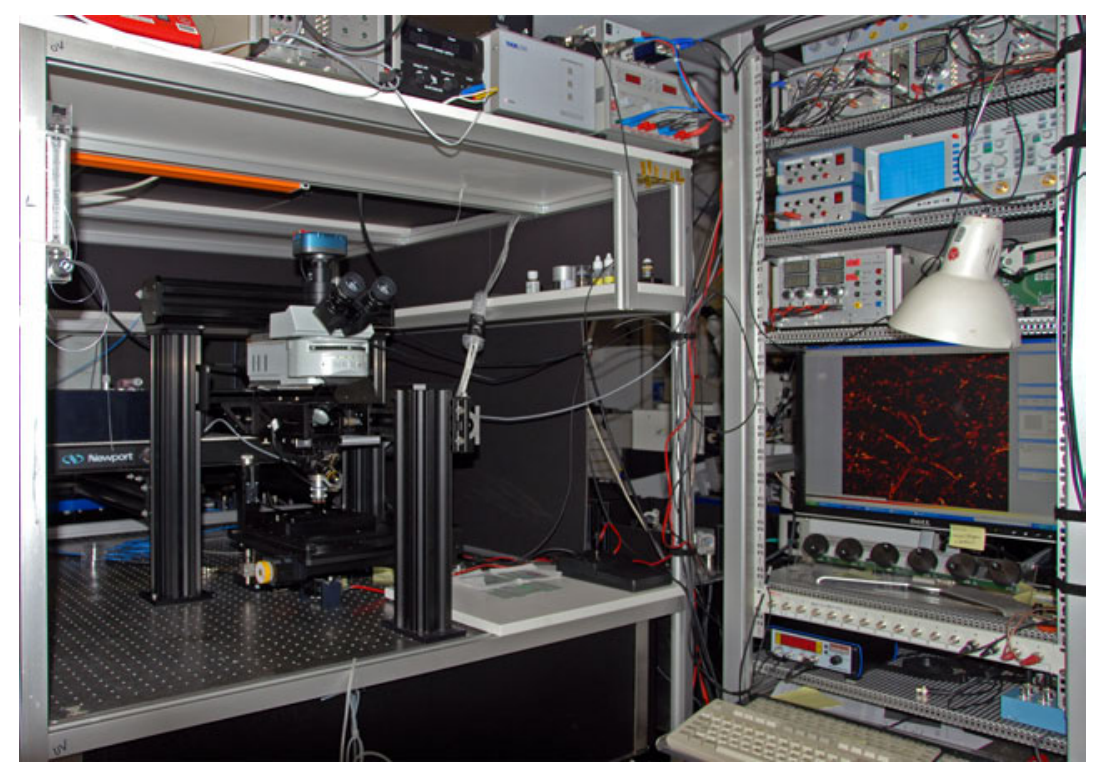

Fig. A.2.1: Overview of the complete laboratory setup built for in-vivo STED microscopy in this thesis. The $\mathrm{PC}$ on the right controls image acquisition. Lasers and optics for beam conditioning and fiber coupling are not depicted.

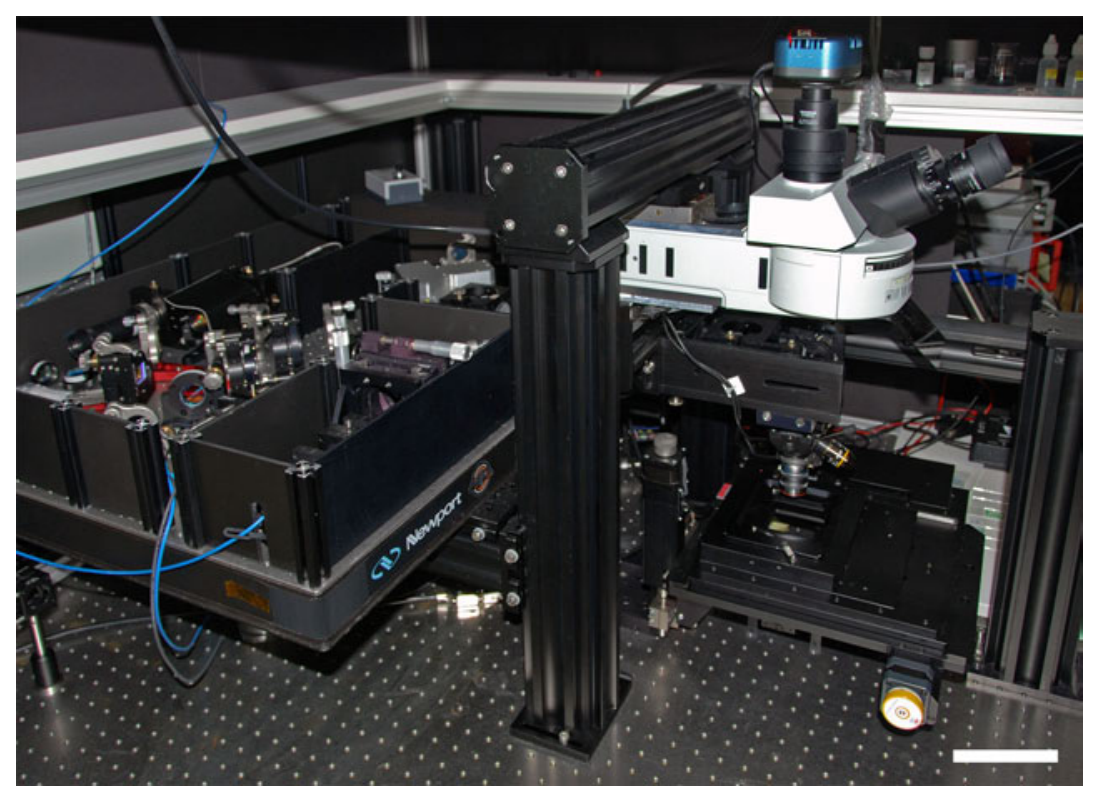

Fig. A.2.2: Perspective showing the microscope optics mounted on a bread board above to optical table. Beam paths continue under the fluorescence condenser from there and are finally deflected towards the microscope objective by a flip-mirror. Laser light is delivered by optical fibers. Scale bar $=10 \mathrm{~cm}$. 


\section{A.3 Confocal single spine recording}
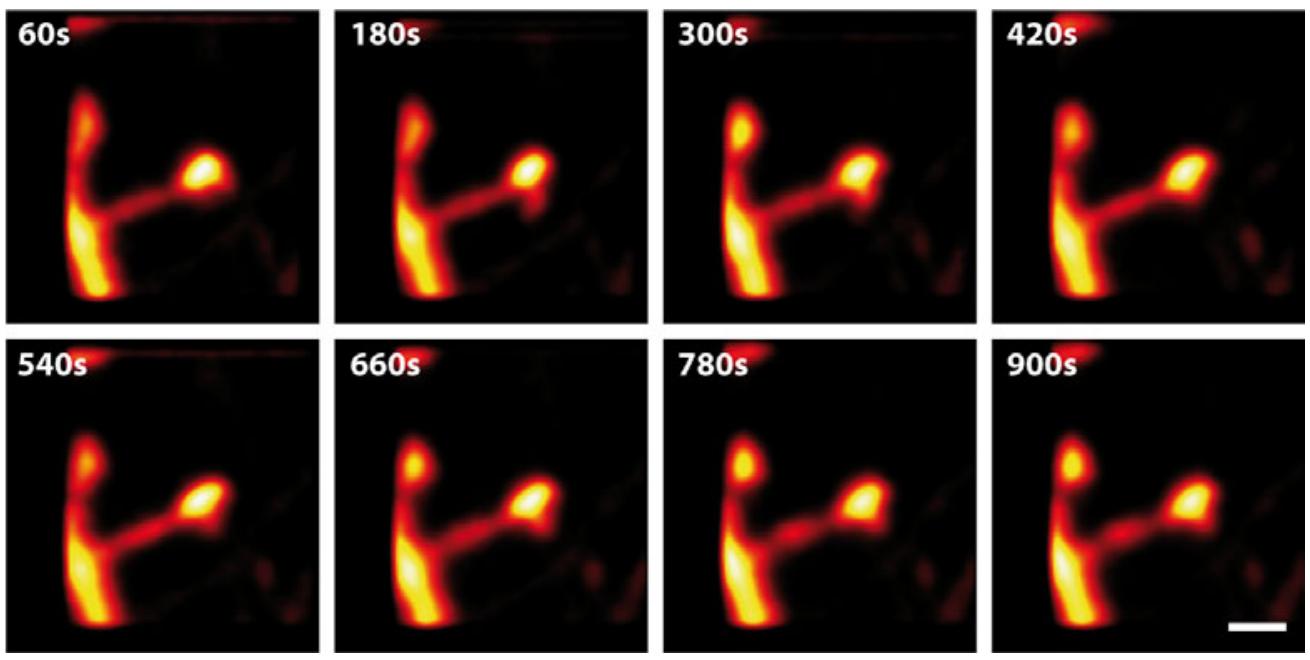

Fig. A.3.1: Before switching on the STED laser, the spine depicted in Fig. 4.4 was imaged at only $1 \mu \mathrm{W}$ excitation power over a timespan of 15 minutes. Stacks consisting of 5 images were recorded every 60 seconds. Even at this low optical power setting, morphological changes at the spine heads can still be observed, albeit at a lower resolution compared to STED. These movements are similar to the ones observed in the STED recordings regarding speed and type, which is taken as an indication that they are not induced by the STED beam. Scale bar $=1 \mu \mathrm{m}$.

\section{A.4 STED resolution obtained in PLP-EGFP mice}

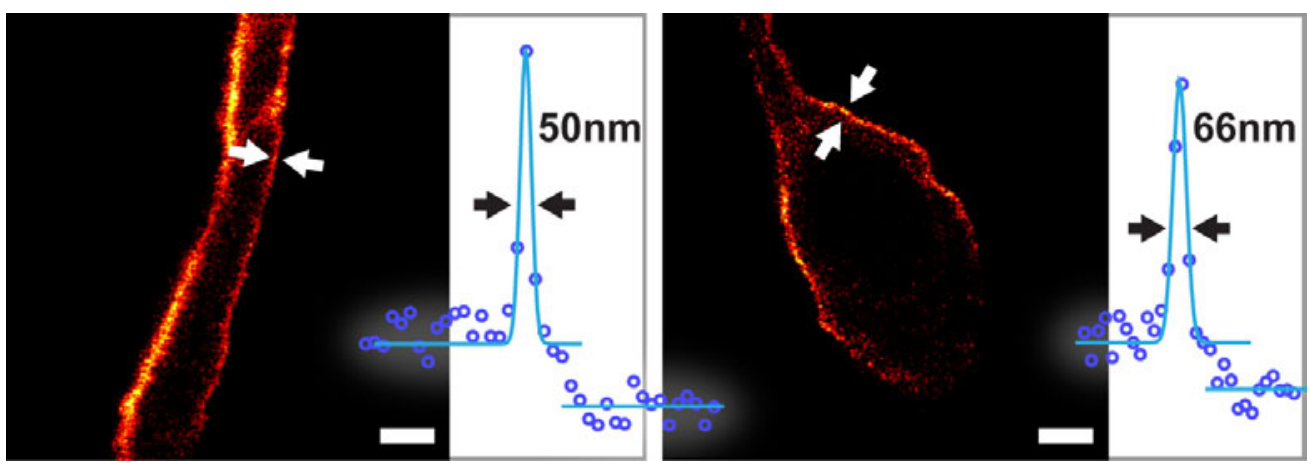

Fig. A.4.1: Despite the lower STED-cross section compared to EYFP at a STED wavelength of 592nm, a 4-fold resolution improvement was routinely achieved for EGFPlabeled oligodendrocytes. Cross-sections of the outer membrane consistently show a FWHM of 50-70nm across different cells. Scale bar $=1 \mu \mathrm{m}$. 
Appendix

\section{A.5 Parameters for in vivo STED recordings}

\begin{tabular}{|c|c|c|c|c|c|c|c|}
\hline Fig. & pixel / nm & \#slices & $P_{e x c} / \mu W$ & $P_{S T E D} / m W$ & $t_{d} / \mu s$ & $\mathrm{depth} / \mu \mathrm{m}$ & mouse age / d \\
\hline Fig.4.2 & $28 \times 28 \times 600$ & 5 & 3 & 27 & 10 & 10 & 65 \\
\hline Fig.4.3 & $25 \times 25 \times 600$ & 5 & 2.3 & 25 & 10 & 10 & 94 \\
\hline Fig.4.4 & $39 \times 39 \times 600$ & 5 & 1.5 & 25 & 10 & 15 & 137 \\
\hline Fig.4.6A & $29 \times 29$ & 1 & 3.8 & 29 & 10 & 10 & 184 \\
\hline Fig.4.6BC & $29 \times 29$ & 1 & 3.2 & 28 & 10 & 15 & 173 \\
\hline Fig.4.9 & $20 \times 20 \times 600$ & 10 & $5.7 / 2.9$ & 24 & $2 \times 10$ & 10 & 93 \\
\hline Fig.4.10 & $20 \times 20 \times 600$ & 10 & $5.3 / 3.5$ & 24 & $2 \times 10$ & 10 & 93 \\
\hline
\end{tabular}




\section{Publications}

Parts of this thesis were published in the following peer-reviewed article:

- S. Berning, K. I. Willig, H. Steffens, P. Dibaj, S. W. Hell. Nanoscopy in a Living Mouse Brain. Science, 335(6068):551, February 2012 


\section{Acknowledgment}

I would like to thank all the people that made this thesis possible and who I enjoyed working with throughout the last years:

- Prof. Stefan Hell for giving me the opportunity to work in a fascinating, interdisciplinary environment and for providing everything that was necessary to make this exciting project happen

- Prof. Christoph Schmidt and Prof. Helmut Grubmüller for their interest and for taking the time to accompany this thesis

- Heinz Steffens, the guy with the calm hands, who contributed enormously to the success of this work and with whom I spent a very enjoyable time in the lab

- Katrin Willig for sharing her experience on live-cell STED microscopy and for her help on the setup and the experiments

- Payam Dibaj for providing the genetically modified mice

- Jan Keller-Findeisen for helping on the numerical studies and for proofreading

- Christian Eggeling for helping with components and for his great collaborative attitude

- Ben Harke, Dominik Wildanger and Volker Westphal for teaching me everything I know about STED microscopy

- Andreas Schönle for providing the software and loads of theoretical insight (on nearly everything)

- Jaydev Jethwa for helping with all kinds of problems in the lab, especially with lasers, and for proofreading parts of my manuscript

- Tanja Gilat, Ellen Rothermel and Rebecca Medda for the preparation of countless biological samples

- Chaitanya Ullal, Nicolai Urban and Mark Bates for critical proofreading of the manuscript

- Ulrike Teichmann and the team of the MPIBPC animal facility for taking good care of our lab animals

- Frank Meyer, Udo Gemm and the whole electronics workshop for the design of custom instruments

- The mechanical, the optical and the carpentry workshops for their craftsmanship

- Antje Erdmann and Michaela Böttcher for taking care of everything related to the GGNB program 
- Donald Ouw for teaching me to master the milling machine and the lathe without suffering the loss of too many fingers

- Sarah Leondarakis, Alena Stephan, Silvia Schirmer and Josephine Stadler for making impossible things happen quickly

- Veronika Müller for being a great fellow around here and taking care of my excess stock of chocolate

- Tanja Brakemann who shared her wisdom on GGNB thesis guidelines with me

- My office colleagues who patiently answered all my trivial questions regarding biology, chemistry and material science and who made this a fun place to work

- All the other people who made my time in Göttingen an unforgettable experience

- Finally, I want to thank my family, that didn't stop me from entering science and always supported me, as well as Julia for her patience and support. 